\title{
Le forum gallo-romain de Feurs (Loire)
}

\author{
par Paul VALETTE* et Vincent GUICHARD **
}

Ia ville de Feurs - l'antique Forum Segusiavorum, chef-lieu de la cité des Ségusiaves - était la première étape importante le long de la voie d'Aquitaine, à $50 \mathrm{~km}$ à l'ouest de Lyon. I a connaissance de l'histoire et de l'urbanisme de la ville antique a largement progressé à la suite de la politique de prévention archéologique mise en place depuis la fín des années 1970. On expose ici la documentation rassemblée sur l'un des plus importants édiffes publics de la ville : le forum. Outre l'identification d'un plan tripartite, classique dans les provinces oc dentales de l'Empire, associant en un ensemble cohérent espace sacré (temple entouré d'une galerie à cryptoportiques), place centrale (bordée de larges portiques abritant des "boutiques») et groupe basilique-curie, 1 : \#r ncipal apport des fouilles récentes est la datation précoce du forum (vers 10/30 après J.-C.), déterminée par dt arguments stratigraphiques. Alliée à la cohérence du projet architectural, cette datation montre que le schtona tripartite était déjà bien implanté en Gaule lyonnaise dès les premières décennies de l'Empire.

On évoque aussi brièvement les principaux résultats acquis sur la trame urbaine de la ville antique, dont le fermin peut être considéré comme le pivot.

Feurs, the ancient Forum Segusiavorum, chieftown of the Segusiavi, was the first important stop on the Roman road leading to Aquitaine, $50 \mathrm{~km}$ west of Lyon. Our knowledge of the history and the urbanisation of the Gallo-Roman town has progressed considerably in recent years, due to systematic archaeological recording since the late 70's. This paper presents evidence for one of the most important public buildings of the town' the forum. Besides the identification of a tripartite plan, already well known in the western provinces of the Roman Empire, the main result from recent excavations is an early date for the forum (ca. 10/30 A.D.), which is based on stratigraphical observations. The perfect coherence of the plan of the monument shows that the scheme of the tripartite forum was already welleslablished in Gallia Lugdunensis in the first decades of the Empire.

The main results obtained about the spatial organization of the Roman town are also discussed, and it appears that the forum is the central point from which the layout of the cily was planned.

Mots clés : forum, urbanisme, gallo-romain, I ${ }^{\mathrm{er}}$ s. après J.-C., Feurs, Loire.

* 6, rue Voltaire, 42110 Feurs.

** Musée Déchelette, 42300 Roanne. 
Nous présentons ici la synthèse des données disponibles sur le forum gallo-romain de Feurs, monument sur lequel nous avons eu l'occasion d'intervenir à plusieurs reprises depuis 1978 à l'occasion de fouilles de sauvetage. La ville est depuis longtemps identifiée au Forum Segusiavorum de Ptolémée et de la carte de Peutinger. Elle est située au centre de la plaine du Forez, à $50 \mathrm{~km}$ à l'ouest de Lyon, au cœur du territoire présumé du peuple ségusiave, le long d'un itinéraire emprunté à l'époque romaine par la voie du réseau d'Agrippa joignant Lyon à Saintes via Clermont-Ferrand et Limoges.

Les premières observations archéologiques précises effectuées sur le site datent d'avant le milieu du $\mathrm{XIX}^{\mathrm{e}} \mathrm{s}$. Elles ne connurent malheureusement pas de suite et il faut attendre la fin des années 1970 pour que des chantiers de sauvetage urbain apportent des données nouvelles sur le sujet. Après celui consacré à l'habitat gaulois, dont les vestiges ont été retrouvés sous le forum (Vaginay, Guichard, 1988), les pages qui suivent constituent le deuxième dossier que nous permettent de livrer dix années d'observations archéologiques sur la ville antique de Feurs.

La documentation disponible sur le forum de Feurs n'est pas définitive, tant s'en faut, les observations ayant été conditionnées par le hasard des opérations d'urbanisme. Néanmoins, le dossier ayant peu de chance d'évoluer dans les années à venir, il nous a paru opportun de ne pas différer plus la publication des données que nous avons rassemblées, qui permettent déjà une restitution en plan presque complète du monument.

Cette mise à plat des données archéologiques justifie sans doute une analyse architecturale plus développée que celle que nous esquisserons. Cette analyse a en fait été conduite parallèlement à l'étude archéologique et a donné lieu à des confrontations fructueuses ${ }^{1}$.

1 M. Pierre André, architecte DPLG, a suivi depuis le début l'évolution du dossier. Nous lui exprimons notre très sincère reconnaissance pour la patience dont il a fait preuve pour tenter d'éclairer notre lanterne sur les problèmes architecturaux posés par les fora. Les résultats de l'étude qu'il a réalisée, qui débordent largement les ambitions de cet article, feront l'objet d'une publication ultérieure. Ils ont par ailleurs donné lieu à la construction d'une maquette de l'élévation, à l'échelle de $1 \mathrm{~cm}$ par mètre, qui est présentée au Musée municipal de Feurs depuis janvier 1990.

Nous devons également remercier nos collègues et collaborateurs sans lesquels cette étude n'aurait pu voir le jour, en premier lieu desquels MM. Michel Vaginay, qui nous a laissé toute latitude pour étudier les résultats d'une fouille de sauvetage réalisée par ses soins en 1981 à l'emplacement de l'égout du forum, et Jean-François Juliaa, qui a participé à toutes les opérations de terrain depuis 1978.

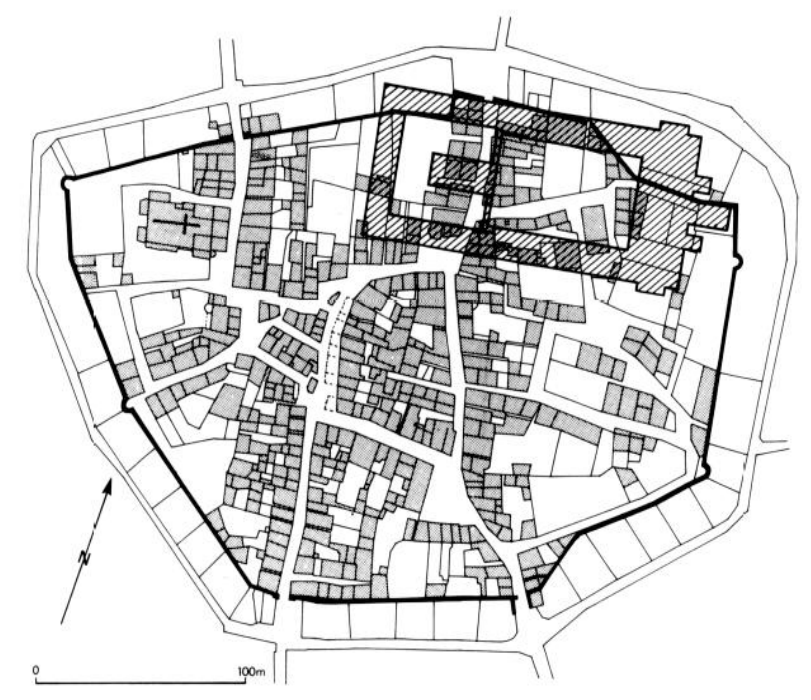

Fig. 1 - Plan de Feurs intra-muros, levé en 1768 par P. Brissat (original conservé au musée de Feurs), montrant en particulier le tracé de l'enceinte de la fin du Xıve s.; on a superposé au plan l'emprise du forum.

À la partie purement documentaire, qui forme l'essentiel de cet article, nous avons également joint une étude comparative et des éléments d'analyse de l'arrière-plan historique de la construction du forum, qui se justifient par la nouveauté que constitue, pour ce type de monument, l'existence à Feurs de critères stratigraphiques précis pour en assurer la datation ${ }^{2}$.

\section{LA REDÉCOUVERTE DU FORUM}

"Cette ville [antique] ayant été absolument détruite, je ne sais de quelle manière, les Comtes de Forez se bâtirent un château sur les ruines d'un vieil édifice, et c'est ce qui fit former peu à peu la ville dans l'état où elle est aujourd'hui». Cette phrase de l'abbé Duguet écrite au début du xviII ${ }^{\mathrm{e}} \mathrm{s}$. (Durand, 1880 , p. 240-241) résume assez bien, quoique de façon laconique, l'évolution urbaine de Feurs depuis l'Antiquité et le rôle qu'a pu jouer l'implantation du forum dans cette évolution. Ses ruines ont en effet servi de support à la première fortification médiévale attestée à Feurs (fig. 1).

Les mentions anciennes des monuments antiques de Feurs ne sont pas nombreuses et ne concernent de manière précise que des découvertes

2 La partie documentaire (p. 112-150) a été rédigée par Vincent Guichard, la partie comparative et historique (p. 151160) par Paul Valette. Les frais occasionnés par cette étude ont été partiellement pris en charge par une aide à la publication allouée par le Ministère de la Culture et par des crédits du Conseil général de la Loire. 
épigraphiques (La Mure, 1674, p. 71-105). C'est l'abbé Duguet qui mentionne «les vieilles ruines romaines" sur lesquelles fut édifié le château et les "couloirs souterrains" que "dans le temps des guerres civiles on pratiqua [...] dans plusieurs caves pour se cacher ou aller au puits de chaque place : telles sont les caves du sieur Jacques Bernard, médecin, à la porte du Palais [extrémité nord de l'actuelle rue du Palais]». S'agit-il d'une allusion au conduit d'assainissement du cryptoportique? Le château, ruiné depuis longtemps à l'époque où il écrivait ces lignes, est mentionné pour la première fois dans une charte de 1246 ("clausuram el castri de Foro»; Chartes du Forez, V, 619). Des textes postérieurs, datés de 1248 (idem, IX, 573), permettent d'entrevoir un ensemble castral modeste, ceint de murailles et de fossés, à proximité duquel sont édifiées quelques maisons ("in burgo et tola villa de Fuer»). Se référant à un terrier de $1473^{3}$, A. Broutin (1867, p. 145-146) localise cet édifice "à l'emplacement du presbytère actuel", l'ancien hôtel de la famille Gaudin au Xvir ${ }^{\mathrm{e}}$ s., au nordest de l'enceinte urbaine du xive s. $^{4}$. Il occupait donc la moitié environ de la zone centrale du forum. La fouille de 1978, située plus au sud, n'a mis en évidence aucune structure pouvant se rapporter à cet ensemble, si ce n'est, à la limite nord de l'aire explorée, une zone de forts remblaiements recoupant les sols antiques et qui peut correspondre à une partie des fossés, comblés au xive $s$. L'installation de nouveaux habitants à l'ouest et au sud de cette enceinte, dès le milieu du xiII ${ }^{\mathrm{e}} \mathrm{s}$, témoigne de son exiguité (Chartes du Forez, IV, 573). Cette occupation est confirmée par des trouvailles monétaires effectuées lors de la fouille de l'îlot de la Boaterie ${ }^{5}$ dans les niveaux qui recouvraient les vestiges du forum. Mais il devait y avoir fort longtemps que dans ce secteur le forum n'était plus qu'un champ de ruines, déjà arasées au niveau où nous les avons découvertes. Les témoignages de la plus ancienne occupation médiévale remontent aux $\mathrm{VI} / \mathrm{IX}^{\mathrm{e}} \mathrm{s}$. (Valette, 1983 , p. 74-75) ${ }^{6}$. Il y avait là une source d'approvisionnement en matériaux de construction de tout

3 Terrier de Feurs renouvelé par Gilberl Jurieu, 14731474, Archives départementales de la Loire, B 2030.

4 Localisation reprise par E. Fournial (Fournial, 1967, p. 56).

5 Des deniers ou oboles d'argent des archevêques de Vienne et de Lyon, un denier de billon du marquisat de Provence (Raimond VI ou Raimond VII). Monnaies inédites.

6 Une agrafe à double crochet, deux plaques-boucles en bronze $\left(\mathrm{vI}^{\mathrm{e}}-\mathrm{VII}^{\mathrm{e}} \mathrm{s}\right.$.), un denier de Louis le Pieux (814) et un denier de Salerne (839-849). premier ordre et il ne fait pas de doute que les maçons médiévaux de Feurs, et certainement aussi leurs successeurs, y ont abondamment puisé. Les comptes de construction des murailles élevées en 1388 (Gonon, 1974) ne mentionnent pas de façon explicite la démolition de murs antiques dans le secteur dı forım ${ }^{7}$. Pourtant l'examen des lambeaux de rempart qui subsistent encore montre de nombreux réemplois de moellons de granite rose d'origine antique. Les éléments de construction en calcaire et en marbre ont dû par ailleurs être systématiquement recherchés, les environs de la ville étant totalement dépourvus de source naturelle de $\operatorname{chaux}^{8}$. Ces murailles de la fin du $x_{I} v^{e}$ s. reprennent en partie, semble-t-il, des fortifications plus anciennes ${ }^{9}$ dont elles auraient réutilisé les matériaux. C'est dans ces dernières que se serait trouvé inclus l'angle nord-est de la curie, assurant ainsi sa conservation jusqu'à ces dernières décennies.

Aussi, ce que l'on peut entrevoir à travers les textes de l'organisation du bourg médiéval laisse présumer que son parcellaire devait peu aux puissantes substructions antiques qu'il recouvrait. La place de la Sarzillerie (aujourd'hui place du Puits de l'Aune), située sur une partie de la branche sud du cryptoportique, est mentionnée dès 1373 dans deux testaments (Fournial, 1967, p. 56). Les ruelles qui s'y rejoignent, dont la rue du Château, antérieure au $\mathrm{xv}^{\mathrm{e}} \mathrm{s}$., ne respectent nullement les orientations qu'aurait pu suggérer le forum. Ce n'est d'ailleurs que très ponctuellement que nous avons pu observer, lors des fouilles de 1978, des tronçons de murs appartenant à des édifices $d u X^{e}$ s. prenant appui sur des maçonneries antiques. En revanche, les structures enterrées du cryptoportique ont été propices à l'installation de nombreuses caves au Moyen Age. Son déblaiement partiel explique les nombreux remplois de calcaire jaune ou blanc dans les façades de maisons anciennes, voire $\mathrm{du} \mathrm{xIX}^{\mathrm{e}} \mathrm{s}$., situées à son emplacement. C'est le cas de certains immeubles bordant l'actuelle rue de Roanne, percée dans les années 1820 , qui longe la façade occidentale du cryptoportique.

7 Ce qui est le cas en au moins un autre point de l'agglomération, puisqu'un certain Jean Fadot est payé "pour abatre et disruyr 20 tezes [ 20 toises, soit environ $25 \mathrm{~m}$ ] de la muralle des Sarazins en les Crotes".

8 Un four à chaux est mentionné dans le terrier de 1473. Le texte de 1388 n'en mentionne pas à Feurs, bien que la construction du rempart ait nécessité près de 300 "charrées" de chaux!

9 "A payé à Janin compaign de Felibert qui ha abattu de la muralle du murs qui sont autour de la ville de Fuer" (Gonon, 1974, art. 322). 
Nous concevons alors clairement que ce monument ait pu rester longtemps oublié, jusqu'à ce que les explorations "souterraines" et la perspicacité de l'abbé Roux, curé de Feurs dans les années 1840, permettent d'en entrevoir l'importance. C'est lui en effet qui a effectué le premier relevé du réseau de galeries d'assainissement du cryptoportique et qui y a reconnu un élément d'un complexe monumental qu'il identifie immédiatement à un forum (Roux, 1851).

Après la publication de Roux, et pour de longues décennies, le passé antique de Feurs retombe largement dans l'oubli par manque d'observateurs possédant la mème clairvoyance que ce pionnier. Il faut attendre 1955 pour voir le dégagement de la partie est du monument (curie et basilique) lors de la création de la place de la Boaterie. La construction de la nouvelle poste, en 1968, permettra de compléter le plan de la partie nord de la basilique.

A partir de 1978, la multiplication des opérations de rénovation dans le centre-ville va provoquer une série d'interventions de sauvetage, conduites le plus souvent dans des conditions difficiles. Néanmoins, ces travaux précisent le tracé du cryptoportique et de son égout, localisent les cellules et le portique de la place centrale du forum ainsi que le podium du temple de l'area sacra, tout en apportant de substantielles indications stratigraphiques. Dernièrement, au printemps 1991, des travaux de réfection de voirie à l'emplacement du forum (place du Puits de l'Aune et rue du Palais) ont permis de vérifier et compléter le plan de l'aire sacrée. Ces travaux n'ayant pu être précédés d'une fouille archéologique systématique, les observations sommaires effectuées à cette occasion ont été intégrées en totalité (et en dernière minute!) au sein de cet article.

Un aperçu des derniers travaux de fouille sur le forum a été donné récemment, dans lequel on avait notamment souligné la possibilité de raccorder de façon cohérente les vestiges monumentaux repérés depuis plusieurs dizaines d'années dans le centre de Feurs dans le cadre d'un complexe monumental s'intégrant parfaitement dans le schéma classique des fora tripartites de l'Occident romain (Vaginay, Valette, 1982). On ne reviendra donc pas sur l'identification des différentes parties du complexe (basilique, curie...), tout en soulignant que les travaux effectués depuis lors (prospection de caves et sondages) ont permis de localiser le temple intégré au forum, dont l'existence avait été alors seulement supposée. On avait également discuté avec un certain détail les indices de datation fournis par la stratigraphie; en l'absence de données nouvelles, on se contentera ici de résumer les conclusions auxquelles avait abouti cette étude. On devra en revanche reconsidérer méticuleusement le problème de la restitution du plan d'ensemble du monument ${ }^{10}$, puisqu'il s'est avéré que les données planimétriques disponibles au moment de la parution de cette première étude étaient nettement trop imprécises pour assembler correctement les différents relevés partiels. De plus, les observations récentes permettent de considérer d'un regard plus critique les renseignements fournis par Roux sur le plan du cryptoportique et qui avaient alors èté utilisés dans la première ébauche de restitution.

\section{LES VESTIGES DU FORUM}

Si l'on met de côté les observations de Roux au siècle dernier, les données archéologiques disponibles sur l'ensemble monumental du forum proviennent de travaux étalés sur les trente dernières années, dont la fiabilité et la précision sont très variables. La reconstitution du plan d'ensemble du monument présente donc de réelles difficultés puisqu'il s'agit de raccorder des relevés effectués à des dates différentes et toujours entachés d'une certaine marge d'erreur. Il sera donc nécessaire de discuter les résultats de chaque campagne de fouille ou de relevés avant d'entreprendre la restitution de ce plan. On décrira successivement, en procédant d'est en ouest, les données disponibles pour l'ensemble basilique-curie, puis la place centrale et ses portiques latéraux, l'aire du temple et son cryptoportique, enfin l'égout d'assainissement du cryptoportique. Certaines des données relatives à ces différentes parties du monument., qui se déduiront de la restitution du plan d'ensemble, n'apparaîtront donc que dans le paragraphe consacré à cette restitution. Les différentes interventions sont localisées sur la figure 2 .

Deux paragraphes complémentaires seront également consacrés aux matériaux utilisés pour la construction et à leur mise en ceuvre, ainsi qu'à la synthèse des indications stratigraphiques de datation.

\section{LA PARTIE ORIENTALE DU forum : la basilique et la curie}

\section{Historique des travaux}

Bien que connus de longue date, puisque inclus dans les fortifications médiévales de la ville, les vesfig. 14 .
10 Également publié dans Gallia, 40, 1982, p. 406 , 
Fig. 2-Localisation des interventions sur les vestiges du forum.

1 : curie et partie centrale de la basilique (fouilles de 1955-56),

2: façade nord de la basilique (fouilles de 1968),

3 : aile sud du portique de la place centrale (fouilles de 1978-79),

4: mur intérieur du cryptoportique (repérage imprécis lors de travaux en 1967),

5 : idem (vestiges apparents dans une cave),

6 : idem (fouilles de 1980),

7 : idem (vestiges apparents dans une cave).

8: mur extérieur du cryptoportique

(vestiges apparents dans une cave),

9 : idem? (vestiges de maçonnerie apparents dans une cave),

10: exèdre (repérage lors de travaux en 1991).

11 : platée de fondation du temple

(fouilles de 1984),

12 : idem (vestiges apparents dans une cave),

13 : platée de fondation du temple et extrémité nord-est du cryptoportique

(repérage lors de travaux en 1991),

14: emmarchement de la place centrale

(vestiges apparents dans une cave).

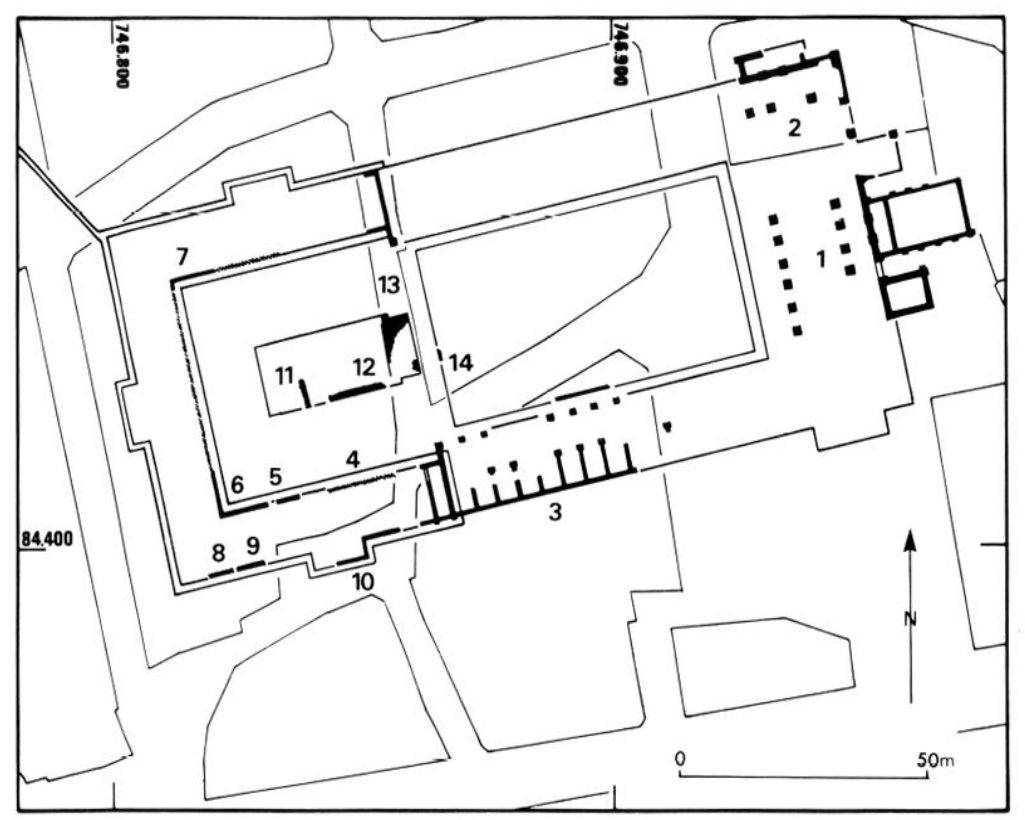

tiges de la curie n'ont fait l'objet d'observations précises qu'au cours des dernières décennies, tandis que les premiers éléments de la basilique commençaient à être dégagés, sans pourtant être identifiés comme tels, au cours des années 1950 .

Le tracé anguleux du rempart médiéval dans sa partie nord-est s'explique par l'annexion d'éléments architectoniques du forum. De ceux-ci, les vestiges de la curie étaient certainement les mieux conservés, puisque le rempart forme à son emplacement un véritable bastion, qui s'explique par l'intégration à la fortification de ses façades est et nord. Roux les décrit déjà dans sa monographie (Roux, 1851, p. 48) : "Il existe, à l'est [du crytoportique], dans le jardin de la cure, deux pans de murs antiques dont les contreforts furent arrachés au moyen-âge. Ils sont construits dans le même système que ceux des égouts [conduits d'assainissement du cryptoportique], en petit appareil et à bain de mortier: les revêtements ont été formés par encaissement, avec des moules mobiles comme ceux du pisé; ils n'ont pas de zones de briques, et indiquent l'époque de la bonne construction. Des travaux exécutés, il y a quatre ans, dans un jardin appartenant à M. Boissonnet, mirent à découvert un petit bâtiment carré, dont l'isolement, l'exiguïté et la disposition me font penser que c'est la cella d'un temple [il s'agit peutètre d'un des édicules disposés de part et d'autre de la curie)". Des photographies prises avant 1952 (voir infra, fig. 10 et 11) ${ }^{11}$ montrent qu'à cette époque le mur est s'élève encore de $2 \mathrm{~m}$ au-dessus du sol et le mur nord d'environ $5 \mathrm{~m}$. La surface qui correspond à la partie intérieure de la curie, abondamment remblayée, est alors occupée par un jardin situé à près de $2 \mathrm{~m}$ en contre-haut des parcelles qui le jouxtent au nord et à l'est. Ce quartier de la ville moderne a subi d'importants réaménagements au cours des années 1952-1956, qui ont conduit au nivellement de ce jardin, au dégagement de la curie (voir infra, fig. 8) et, malheureusement, à l'arasement partiel de ses vestiges $^{12}$ (fig. 2, point 1). Plusieurs personnes se sont alors efforcées d'enregistrer les découvertes archéologiques au fur et à mesure de l'avancement des travaux (notamment MM. J. Gorce et G. Brassart), sans que puisse véritablement être organisée une fouille de sauvetage. Il subsiste de ces observations un plan d'ensemble des structures dégagées (fig. 3), dû à $\mathrm{J}$. Gorce, une demi-douzaine de photographies et des indications succinctes sur la stratigraphie et le mode de construction de l'édifice, dispersées dans quelques notes publiées (Gorce, 1957; Gallia, 14, 1956 , p. 279 et 16,1958, p. 364$)$ et archives inédites ${ }^{13}$.

11 Archives du Musée municipal de Feurs.

12 Malgré leur inscription à l'inventaire supplémentaire des Monuments historiques depuis 1929.

13 Archives de la Direction régionale des Antiquités historiques. 


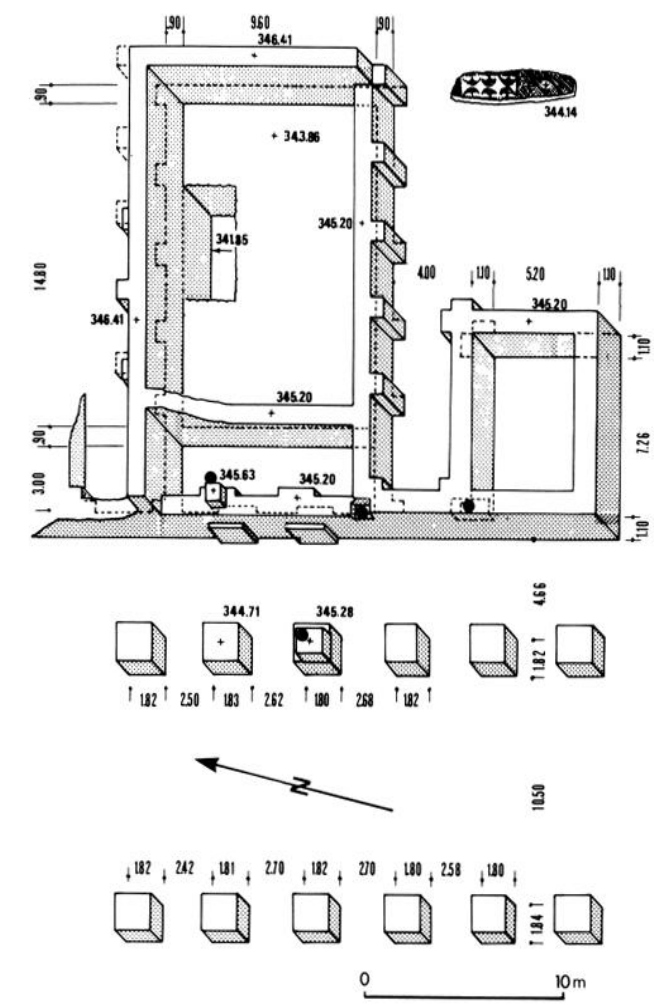

Fig. 3 - Plan des vestiges de la curie levé en 1956 par J. Gorce (fig. 2, point 1); les cotes d'altitude relatives ont été restituees selon le nivellement $\mathrm{VGF}$; les pastilles noires indiquent l'emplacement de blocs de grand appareil en calcaire jaune.

Ces travaux ont essentiellement permis de relever un plan d'ensemble de la curie et de plusieurs autres vestiges appartenant visiblement à la même époque de construction. Il s'agit :

- à l'ouest, d'une dizaine de piles de fondation ordonnées en deux travées parallèles à la façade de la curie, appartenant en fait à la péristasis de la nef centrale de la basilique;

- à $4 \mathrm{~m}$ au sud de la curie, d'un bâtiment plus petit dont le côté ouest s'appuie sur le même mur que celle-ci.

Deux importants fragments d'une mème mosaïque à décor géométrique noir et blanc ont également été dégagés à quelques mètres au sud de l'angle sud-est de la curie (Stern, Blanchard-Lemée, 1975).

Le prolongement de l'ensemble monumental vers le nord a ensuite été hâtivement exploré par une campagne de sondages en 1968, au moment de la construction du bâtiment de la nouvelle poste (travaux de S. Boucher; Gallia, 24, 1971, p. 411) (fig. 2, point 2). Cette exploration a été menée à partir d'étroites tranchées orthogonales, très espacées, ce qui rend très malaisée l'interprétation des vestiges dégagés (fig. 4). Inconvénients supplémentaires : les vestiges sont arasés extrêmement bas, puisque leur sommet apparaît à une cote très nettement inférieure à celle de la base de l'élévation de la curie (ce qui s'explique par la localisation à cet emplacement du fossé de la fortification médiévale), et les seuls documents disponibles sont des relevés de géomètre à l'état brut, sur lesquels les orientations des parements, certainement peu lisibles à partir des fondations dégagées, ont été manifestement mal transcrites, et les cassures accidentelles souvent mal distinguées des lignes de parement. On sera donc dans l'obligation d'interpréter les observations consignées sur ce plan, en prêtant beaucoup plus attention aux points cotés, sûrement positionnés, qu'au tracé lui-même. Ces sondages ont du moins permis de trouver la limite nord des deux travées de piles situées à l'ouest de la curie, ainsi que plusieurs autres éléments de maçonnerie difficilement interprétables en tant que tels, mais qui appartiennent certainement aux fondations de la basilique (cf. infra).

Enfin, en 1988, deux sondages limités ont été opérés de part et d'autre du mur est de la curie pour préciser les données de nivellement et de stratigraphie, points sur lesquels les travaux antérieurs ne fournissent que des indications très sommaires (voir infra, fig. 9).

\section{Synthèse de la documentation}

\section{Basilique}

Le mur de façade est de la basilique a été dégagé en même temps que la curie qui y est accolée. Seul vestige de l'édifice actuellement visible, la partie médiane de ce mur présente une épaisseur de $1,1 \mathrm{~m}$, et est construite en petit appareil assez irrégulier. Elle présente d'autre part deux renforcements de même module que les piles de la péristasis, distants de $2,0 \mathrm{~m}$, de part et d'autre de l'axe de la curie (fig. 4).

Les piles dégagées devant la curie, qui appartiennent aux grands côtés de la péristasis de la nef, aujourd'hui arasées et recouvertes, ont une dimension de 1,81 sur $1,81( \pm 0,01) \mathrm{m}$ (fig. 5 et 6$)$. La distance de l'axe de la première travée au mur de façade est de la basilique est de $5,5 \mathrm{~m}$, tandis que l'écartement des deux travées, d'axe à axe, est de $12,3 \mathrm{~m}$. Au sein d'une même travée, l'écartement des piles est assez irrégulier : il varie de $2,4 \mathrm{~m}$ (soit $4,2 \mathrm{~m}$ entre axes) à $2,7 \mathrm{~m}$ (4,5 m entre axes). Les trois piles supplémentaires dégagées en 1968 appartiennent au petit côté de la péristasis, qui en comportait quatre à 


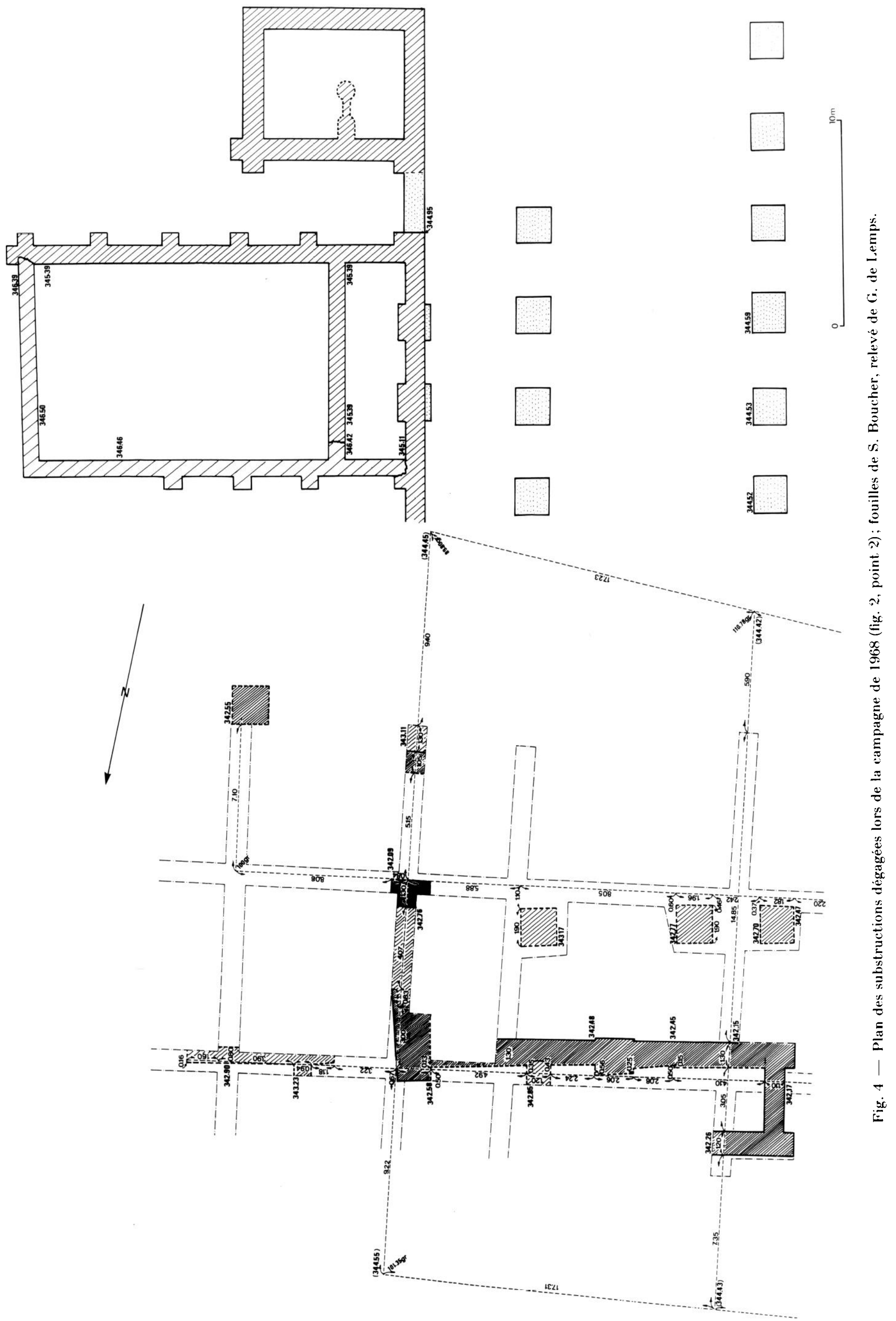




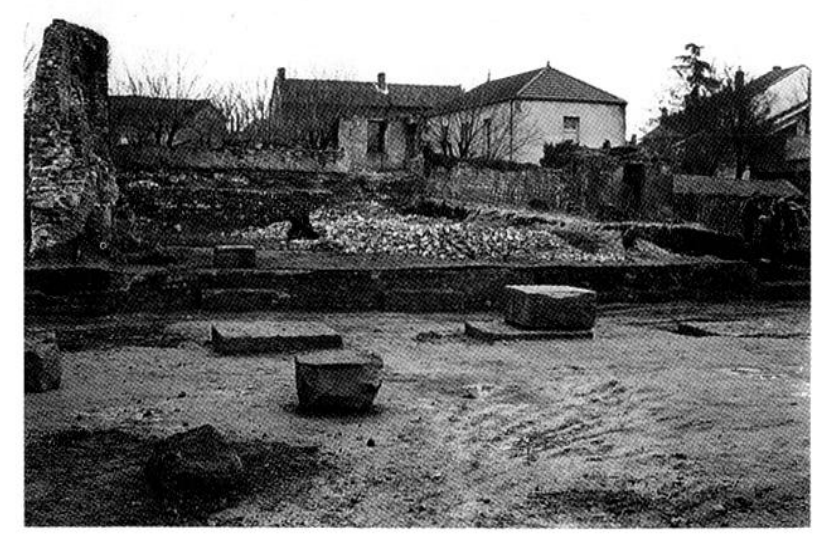

Fig. 5 - Vue depuis l'ouest de la façade ouest de la curie et. des piles de la péristasis de la basilique pendant leur dégagement, en 1955-1956. On remarque en particulier deux blocs de soutènement de colonnes en grand appareil subsistant dans leur position d'origine, l'un sur une pile de fondation de la péristasis, l'autre sur le mur ouest de la curie.

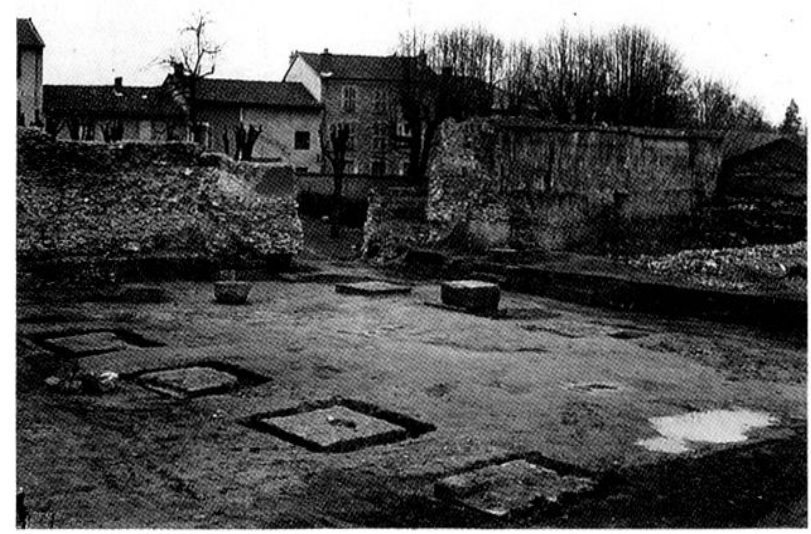

Fig. 6 - Vue depuis le sud-ouest de la façade ouest de la curie et des piles de la péristasis de la basilique pendant leur dégagement, en 1955-1956.

l'origine. Elles indiquent une largeur de la nef de $12,5 \mathrm{~m}$, cohérente avec les observations antérieures, et divisée en trois espaces de $4,15 \mathrm{~m}$.

La façade ouest de la basilique n'a jamais été repérée, ce qui nous empêche de comprendre l'articulation de l'édifice avec la place centrale du forum (colonnade ou mur continu percé d'ouvertures plus espacées). Son emplacement est cependant restituable précisément par symétrie. La façade nord du bâtiment a en revanche été partiellement dégagée en 1968, mais les relevés sont d'interprétation difficile. Ils montrent l'existence d'un massif de maçonnerie très large dans le prolongement du mur ouest de la curie, avec un vide intermédiaire que l'on doit mettre, sans doute possible, au compte de destruc- tions médiévales (fossé de l'enceinte urbaine). L'extrémité des murs latéraux de la nef serait donc très élargie $(1,8 \mathrm{~m}$ contre $1,1 \mathrm{~m}$ dans leur partie centrale) et terminée par un contrefort massif $(1,0 \times 1,8 \mathrm{~m})$, caractéristiques qui s'expliquent par le fait que ces maçonneries jouent le rôle de massifs de culée pour l'ensemble de l'édifice.

Ce mur se raccorde avec un autre, perpendiculaire, large de $1,1 \mathrm{~m}$, qui est manifestement le mur de façade nord, ce que confirme sa distance à la péristasis $(5,5 \mathrm{~m})$, identique à celle relevée sur la façade est de l'édifice. Une cellule supplémentaire (dimensions dans ceuvre : $3,0 \times 11,0 \mathrm{~m}$ ) vient se greffer sur la façade extérieure de ce dernier; ses parois latérales sont situées dans l'axe des travées de la péristasis. Deux épaississements irréguliers du mur de façade nord dans sa partie centrale doivent ètre considérés comme les fondations de deux piles engagées, identiques à celles placées de part et d'autre de l'axe de la curie dans le mur est. Leur écartement, légèrement inférieur à celui des piles de la péristasis, permet d'ailleurs de penser que l'élévation de cet exèdre pouvait être identique à celle du compartiment intermédiaire entre la basilique et la curie, dont les dimensions en plan sont très proches.

\section{Curie et bâtiments annexes accolés à la façade est de la basilique}

La documentation à l'égard de la curie est nettement plus complète que celle disponible pour la basilique, ce qui s'explique par son meilleur état de conservation. L'ensemble du bâtiment est actuellement visible, mais son remblaiement ne respecte absolument pas les nivellements d'origine (fig. 7, 8).

Ses dimensions hors œuvre sont de 11,4 sur $20,6 \mathrm{~m}$ (contreforts non compris). Ses trois murs est, nord et sud ont une largeur de $0,9 \mathrm{~m}$, la fondation du mur est étant plus massive $(1,2 \mathrm{~m}$ de largeur $-\mathrm{cf}$. fig. 9). D'après un sondage effectué dans les années 1950 , la profondeur des fondations est d'environ $2 \mathrm{~m}$. À chacun de ces murs sont accolés des contreforts de $0,9 \mathrm{~m}$ de largeur également, saillants de $0,6 \mathrm{~m}$, et distants de $2,5 \pm 0,2 \mathrm{~m}$. Les façades latérales en comportent cinq, la façade arrière (est) quatre; trois d'entre eux et les deux de l'extrémité est de la façade nord ont été arasés au Moyen Age, mais leur empreinte reste bien visible dans le parement. À l'extrémité ouest des façades latérales, le contrefort d'angle est engagé dans le mur est de la basilique; son écartement du contrefort suivant est nettement plus large $(4,0 \mathrm{~m})$. La face extérieure de ces trois murs et les contreforts sont montés en petit appareil très régulier, avec joints en ruban, tandis 
Fig. 7 - Plan et profils est-ouest de la curie.

A-A : élévations actuelles et stratigraphie de la basilique vues depuis le sud (1: niveas: d'occupation protohistorique, scellé par le "sol primitif", qui correspond au nivellement au moment de l'implantation du bàtiment: 2 : couche de remblai non décrite par les fouilleurs; 3: couche de remblai constituée d'éclats de calcaire jaune tassés):

B-B' : restitution des niveaux de circulation dans l'axe longitudinal du bâtiment:

$C^{\prime}-C$ : élévations actuelles vues depuis le nord: la partie grisée correspond à une partie de l'élévation arasée pendant les années 1950 et restituée d'après photographies.
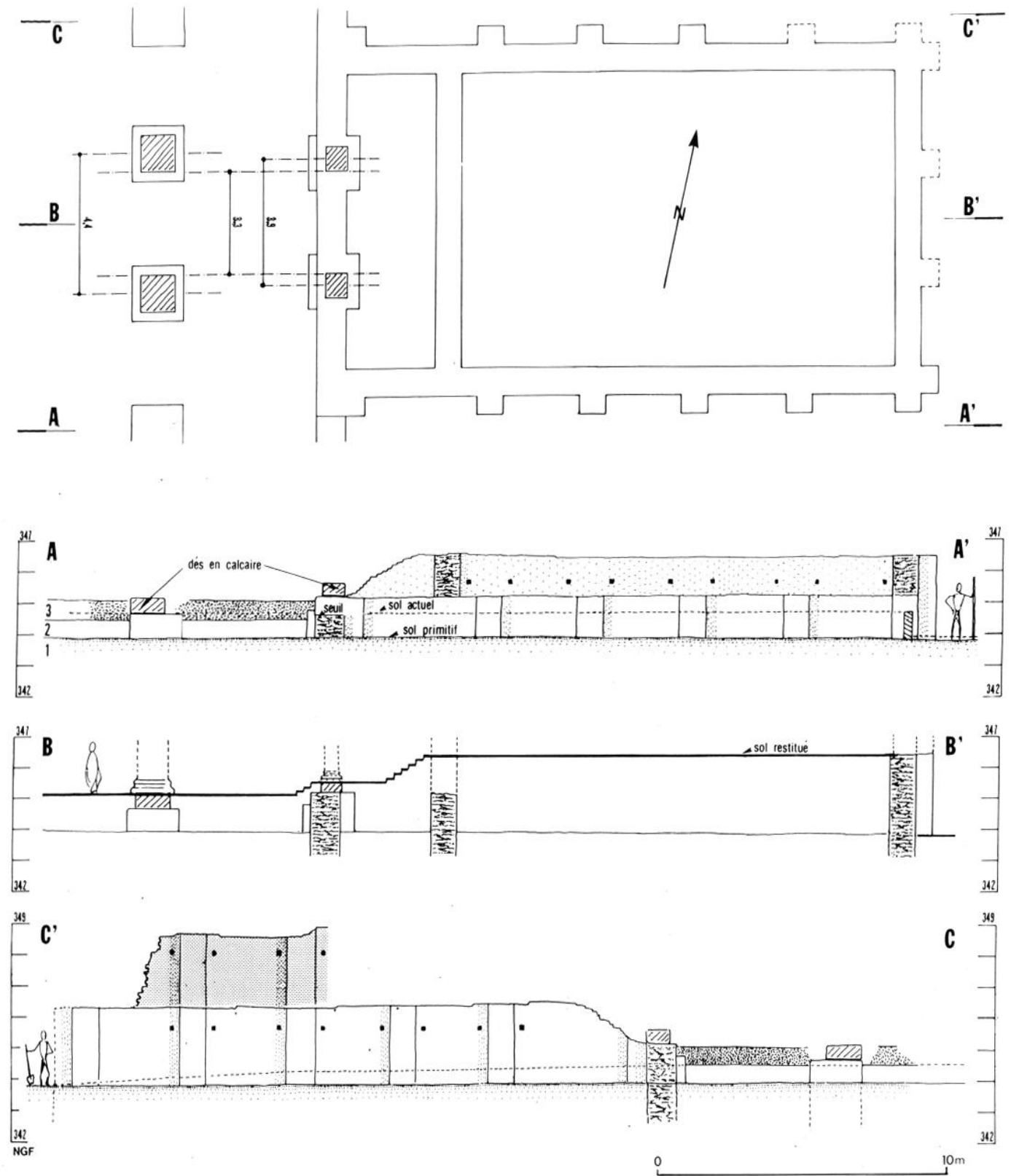

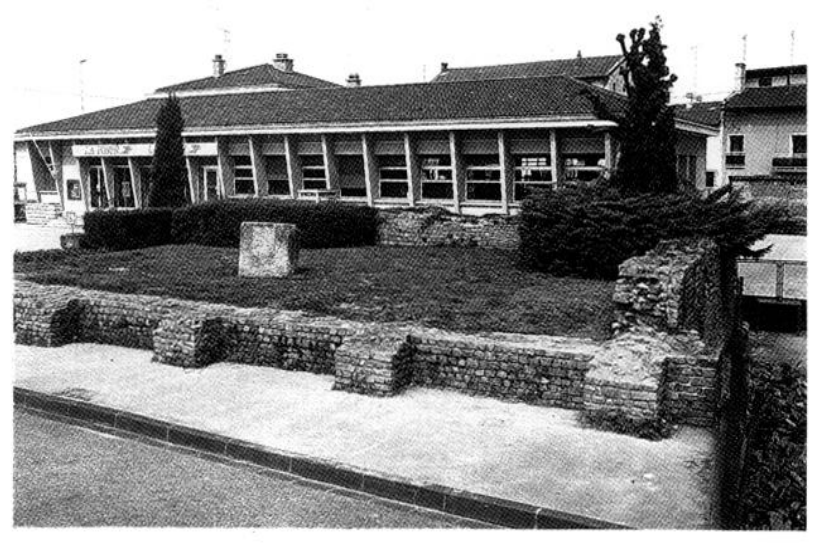

Fig. 8 - Vue générale de la curie depuis le sud-est (1988). que sur la face interne, si le mème rythme des assises est respecté, les moellons utilisés sont plus grossièrement équarris et le jointoyage plus hâtif. On a pu également remarquer tous les $0,6 \mathrm{~m}$ environ (soit 5 ou 6 assises) l'existence d'un joint plus épais qui dénote le raccord entre deux "journées de travail" (fig. 9).

Le mur de la façade ouest de la curie, qui est également le mur de façade est de la basilique, est sensiblement plus épais $(1,1 \mathrm{~m})$ et présente deux renforcements régulièrement espacés de part et d'autre du grand axe de l'édifice, de même module que les piles de la basilique $(1,8 \times 1,8 \mathrm{~m})$, et qui, sur le côté ouest, ne s'élèvent pas plus haut que le sommet de ces piles. Ce mur est en petit appareil 
irrégulier sur ses deux faces; d'après J. Gorce, un important bloc de calcaire jaune était inclus dans son parement, à proximité de l'angle sud-ouest de la curie (fig. 3). Un dé du même calcaire (module: $0,80 \times 0,80 \times 0,42 \mathrm{~m}$ ), présentant un trou de louve sur sa face supérieure, mieux travaillée, et des traces de feu, repose encore sur une des piles engagées dans ce mur, dans une position qui semble d'origine.

L'espace intérieur de la curie est cloisonné par un mur de refend nord-sud de $0,9 \mathrm{~m}$ d'épaisseur qui
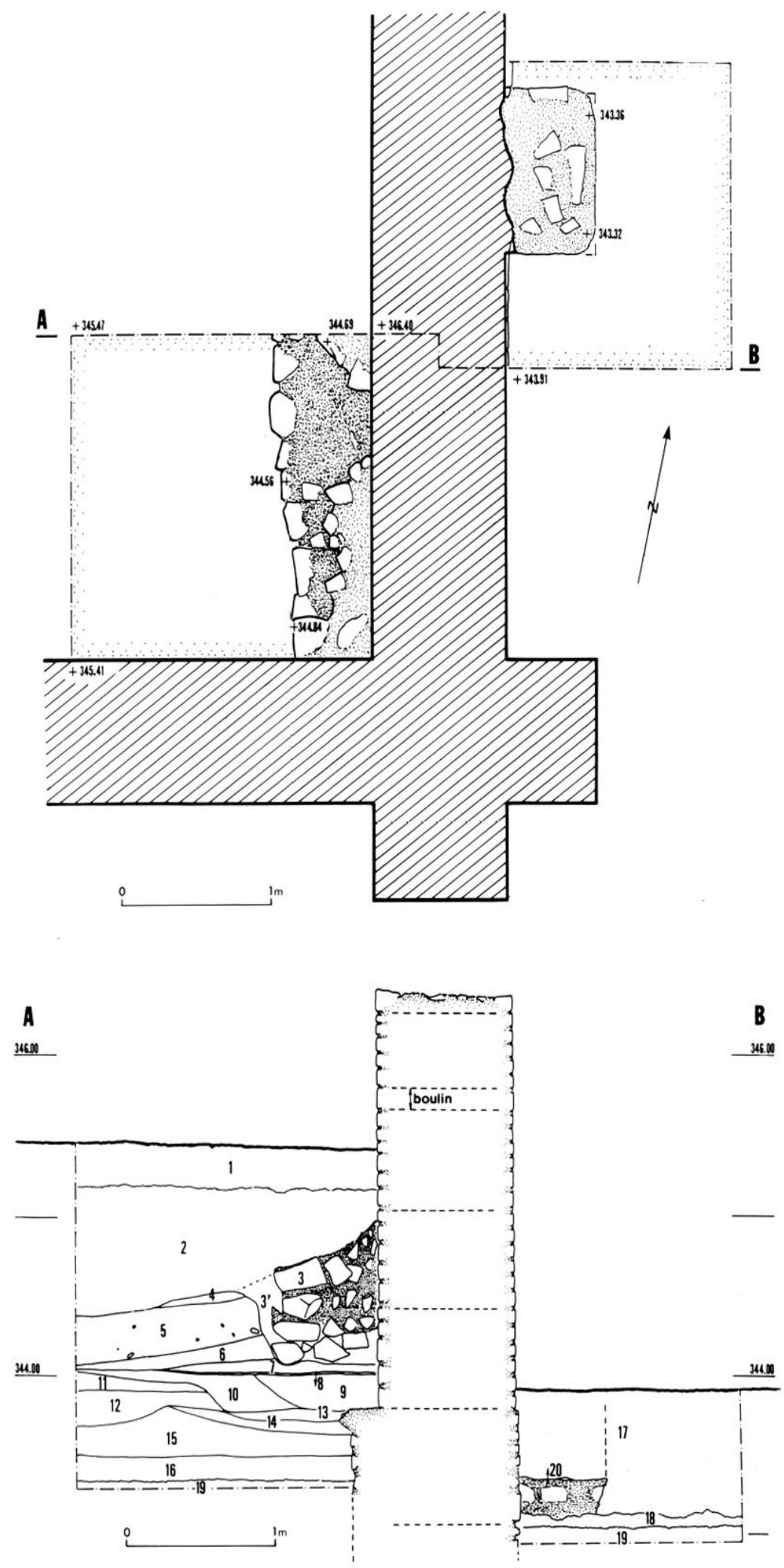

Fig. 9 - Stratigraphie du remplissage interne de la curie, dans l'angle sud-est (sondage de 1988).

$1:$ terre végétale ;

2 : couche hétérogène brune, gravillonneuse (remblai moderne);

3 : fondation de mur liée avec un mortier blanc pulvérulent (médiéval);

$3^{\prime}$ : tranchée de fondation du mur 3 :

4 : terre gris sombre tassée

(sol de travail lié à la construction de 3); 5 : gravier jaune grossier (remblai antique);

6 : gravier gris, fin (remblai antique); $7:$ gravier jaune, fin (remblai antique); 8 : sable blanc (apport par ruissellement); 9: gravier ocre, fin (remblai antique); 10 : gravier ocre, grossier (remblai antique);

11: terre brun clair, gravillonneuse (niveau protohistorique remanié);

12 : terre brune, gravillonneuse (niveau protohistorique remanié); 13 : terre grise, avec éclats de granite rose et galets (sol de travail);

$14:$ terre gris sombre tassée (sol de travail);

15 : terre brune, gravillonneuse (niveau d'occupation protohistorique); 16 : terre gris sombre, à inclusions fines; 17 : remblai hétérogène sombre, limoneux (moderne ou médiéval);

18 : épandage de mortier (médiéval ?);

19 : substratum (gravier ocre, fin);

20 : base de la fondation d'un contrefort arasé du mur de la curie. 
délimite un premier compartiment très ètroit à l'ouest (3,0 × 9,6 $\mathrm{m}$ dans œuvre) et une grande salle de 14,8 sur 9,6 m. Ce mur est actuellement arasé à la cote $345,20 \mathrm{~m}$ NGF environ, mais une élévation supplémentaire jusqu'à la cote 346,50 est discernable sous forme d'un arrachement contre la paroi interne du mur de façade nord. D'une façon plus générale, le niveau d'arasement des murs de la curie est partout situé à une cote comprise entre 345,00 et 346,50 , soit de $1,70 \mathrm{~m}$ à $3,15 \mathrm{~m}$ au-dessus du niveau du sol primitif (343,80 à l'est du bâtiment), tel qu'il a été retrouvé dans les sondages de 1988, ce sol "primitif", qui scelle des niveaux d'occupation plus anciens, correspondant à l'état du nivellement au moment de l'implantation du forum. Les photographies anciennes (fig. 10,11) permettent néanmoins de restituer une élévation supplémentaire de $2,7 \mathrm{~m}$ environ pour le mur de façade nord, soit une élévation totale proche de $6 \mathrm{~m}$; ce mur présenterait ainsi deux rangées de trous de boulin, l'une, encore visible, à $1,9 \mathrm{~m}$ audessus du sol primitif, l'autre environ $2,4 \mathrm{~m}$ au-delà. Les sondages de 1988 (fig. 9) montrent que le sommet des fondations affleure le niveau du sol primitif, niveau auquel on trouve également des sols de travail caractérisés (éclats de granite rose et épandages de mortier) liés à la construction du bâtiment. Le niveau du sol extérieur correspondant à la période de fonctionnement du monument ne devait être guère plus élevé que le sol primitif, puisque l'élévation en petit appareil commence à son niveau. En revanche, on a mis en évidence à l'intérieur du bâtiment des remblais de graviers stériles qui surmontent directement les sols de travail et sont conservés sur une épaisseur de $1 \mathrm{~m}$; ils indiquent que le sol de la curie était nettement plus élevé que le niveau de circulation extérieur. Un autre argument en ce sens, tout aussi probant, est le niveau d'arasement actuel de la curie, qui exclut l'existence d'un seuil en deçà de la cote 345,00 .

L'édicule sud, aujourd'hui arasé et remblayé, n'est connu que par les relevés de J. Gorce. Il est séparé de la curie par un espace de $4,0 \mathrm{~m}$. Ses dimensions dans œuvre sont de 5,20 sur $7,26 \mathrm{~m}$ (proportion de $1: \sqrt{ } 2$ ). Ses murs ont tous une épaisseur de $1,10 \mathrm{~m}$. Deux contreforts saillants de $0,6 \mathrm{~m}$ ont été relevés à son angle nord-est; il est logique d'en restituer deux semblables à l'angle sud-est. Les faces extérieures des murs est, sud et nord sont en petit appareil régulier. Les relevés indiquent également la présence d'un important fragment de calcaire dans l'appareil du mur ouest, tandis que sur certains seulement, et bien que jamais mentionné dans les documents de l'époque, un massif de maçonnerie énig-

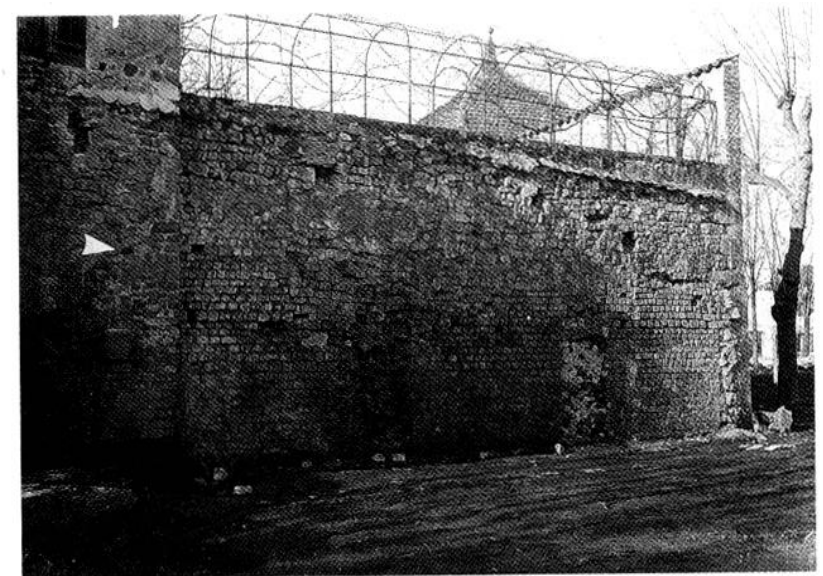

Fig. 10 - Façade est de la curie avant son arasement, en 1955-1956 (la flèche indique le niveau d'arasement actuel).

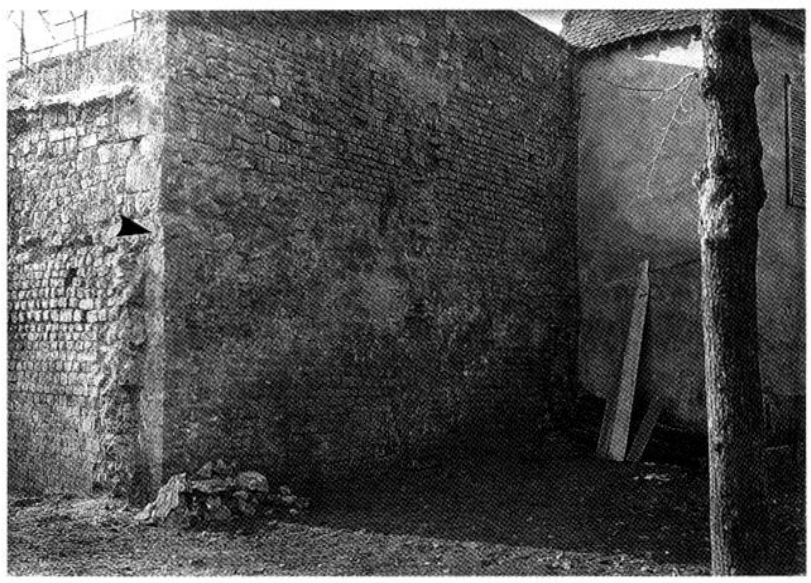

Fig. 11 - Angle nord-est de la curie avant son arasement, en 1955-1956 (la flèche indique le niveau d'arasement actuel).

matique est figuré à l'intérieur de la construction (en pointillé sur la fig. 4).

Bien que non indiqué sur le plan de J. Gorce (fig. 3), un seuil de 2,8 $\mathrm{m}$ de large permettait d'accéder à la basilique de l'extérieur, par l'est, entre la curie et l'édicule sud : il est clairement attesté par la prolongation du parement du dernier contrefort de la partie occidentale de la façade sud de la curie sur toute l'épaisseur du mur est de la basilique (détail encore visible à l'heure actuelle). Il devait être situé à la cote 344,6 environ d'après les photographies de fouille.

Au nord de la curie, le relevé ancien indique un départ de mur est-ouest qui paraît appartenir à un édicule symétrique de celui dégagé au sud du bâtiment. D'autre part, les sondages de 1968 ont repéré un massif de maçonnerie très arasé qui correspond à première vue à une pile de fondation de même 
module que celles de la basilique, mais dont l'emplacement correspond précisément à celui de l'angle nord-est de l'édicule supposé : ce massif peut avec vraisemblance être identifié aux fondations de ce bâtiment. Enfin, on notera qu'il n'existe pas de seuil donnant accès à la basilique entre la curie et cet édicule nord.

Les fouilles de 1968 ont également permis de dégager un segment de mur est-ouest dans le prolongement est du mur de façade nord de la basilique, large de $0,9 \mathrm{~m}$. Sa fonction ne peut évidemment pas être déterminée. Il témoigne du moins de l'existence d'aménagements annexes importants à un emplacement situé au-delà de la limite de l'enceinte du forum, mais malgré cela inclus dans la même insula que le monument, et qui devaient enclore la curie et les deux édicules situés de part et d'autre. On peut penser à un enclos ceinturé de portiques, encadrant la curie, comme c'est le cas à Clunia, par exemple (De Palol, 1987, fig. 5).

\section{Nivellement}

La cote du sommet des piles de la basilique dégagées dans les années 1950 se situe entre 345,52 et 345,59 . L'une d'elles servait encore de base à un dé de calcaire jaune, aujourd'hui déplacé dans l'intérieur de la curie, et qui était alors sans doute approximativement dans sa position d'origine (mais décentré par rapport à la pile). Ses dimensions sont de 1,2 sur 1,2 sur $0,5 \mathrm{~m}$. Seule sa face supérieure est correctement travaillée. les faces latérales étant très grossièrement amaigries et donc non visibles. Les documents conservés indiquent d'autre part que les piles maçonnées sont appareillées en petits moellons assez réguliers sur $0,75 \mathrm{~m}$ à partir de leur sommet, et qu'elles s'enfoncent dans le sol d'au moins $1 \mathrm{~m}$ supplémentaire. Ils notent aussi que les piles ont été retrouvées "sous une aire composée de pierres disposées en hérisson entre deux couches d'un agglomérat de terre ocre" (Gorce, 1957, p. 60), aire qui, selon un autre document, est décrite de la façon suivante: "béton jaune", "la pierre de ce béton et celle des dés sur les piles sont scmblables", "60 cm en trois couches bien organisées; lui et les piles devaient avoir une résistance énorme, [...] on aurait pu installer un marteau pilon...) (G. Brassart, lettre au Directeur des Antiquités: 8 juillet 1956). On connaît la cote du sommet de cette couche par ce dernier document : le dé en place sur une pile de la basilique émergeait de $0,13 \mathrm{~m}$ au-dessus, et celle posée sur le mur de la curie de $0,41 \mathrm{~m}$ (soit donc une cote approximative de 344,95 ) ; il est également indiqué qu'elle vient buter contre le mur est de la basilique et que ses limites primitives à l'ouest et au sud ont disparu. Cette couche, qui est finalement assez bien décrite, paraît avoir été de nature identique à des horizons retrouvés à l'emplacement des portiques latéraux du forum (cf. infra) et interprétés comme des remblais destinés à exhausser le sol au niveau de circulation définitif, et dans lesquels l'alternance de couches tassées de débris de calcaire jaune (déchets de taille?) et d'épandages de mortier conduit par endroits à un sol extrêmement compact. Cette couche a donc tout lieu d'être liée à la construction du monument. Un autre argument renforce ce point de vue : on peut en effet noter qu'au niveau d'un raccord de maçonnerie situé à la cote 345,05 dans le mur ouest de la curie, on remarque un épandage de calcaire jaune broyé emprisonné dans l'épaisseur de ce mur, comme si le remblai en question avait été mis en place avant l'érection du mur au-dessus de sa fondation, rendant ainsi possible le dépôt de salissures de même origine sur une surface d'attente restée à l'air libre quelque temps. Il est de plus remarquable que la hauteur de la partie émergeante du dé en place sur une pile $(0,13 \mathrm{~m})$ laisse tout juste la place pour un dallage, niveau qu'il affleurerait, comme attendu s'il s'agit effectivement d'une base de soutènement de colonne.

Le niveau du sol primitif doit logiquement s'identifier à celui de la base de la partie appareillée des piles, soit à la cote 343,8 , qui correspond parfaitement à celle relevée plus à l'est en 1988. Il reste alors à supposer l'existence d'une couche de remblai supplémentaire d'une épaisseur de $0,6 \mathrm{~m}$ entre le sol primitif et le sol de «béton jaune» et dont la description a été omise.

Le sol de la basilique était donc situé à environ $1,15 \mathrm{~m}$ au-dessus du sol primitif. On ne pouvait accéder à la curie que par une volée de marches supplémentaires, dont l'emplacement est en fait facile à déterminer (fig. 7, coupe BB'). Il est en effet clair que le seul accès à la curie se faisait par la basilique : les deux piles engagées dans son mur de façade ouest fournissent l'emplacement de cette ouverture, centrée sur le petit axe de la basilique. I.e mur de refend de la curie donne alors nalurellement l'extrémité supérieure de cet escalier par lequel on accédait, par une nouvelle ouverture, à la salle principale. I,'espacement réduit de ces deux piles engagées par rapport à celles de la péristasis, additionné à l'existence d'un dé de calcaire en place sur l'une d'entre elles, permet de dissimuler exactement les montants de cette ouverture, d'une largeur de $3,9 \mathrm{~m}$, derrière les colonnes centrales de la péristasis (fig. 7). Cette disposition ménageait de plus deux alvéoles latérales de 3,0 sur 3,0 m de part et d'autre de l'escalier. 
Il est plus difficile d'estimer le niveau de circulation exact de la curie. La longueur de l'escalier d'accès $(4 \mathrm{~m})$ fixe du moins une hauteur maximale possible à la cote approximative de 346,5 (soit $1,5 \mathrm{~m}$ au-dessus du niveau de la basilique). De fait, sur certaines photographies de fouille anciennes, on discerne assez nettement un décrochement du parement interne du mur nord à la cote 346,50 , qui est le niveau d'arasement actuel de ce mur. On retiendra done cette cote comme niveau probable du sol de la curie, qui serait ainsi situé $2,7 \mathrm{~m}$ au-dessus du sol primitif.

\section{Données stratigraphiques}

Les fouilles des années 1950 et de 1968 ne paraissent avoir livré presque aucun mobilier archéologique. On mentionne seulement en 1956 la découverte d'un petit dépôt de marbres près de l'angle sud-est de la curie, dont deux plaquettes inscrites de graffili, l'une conservée au musée de Feurs; l'inscription qu'elle porte semble correspondre à une formule votive (Gallia, 14, 1956, p. 281, fig. '22). De la mème époque date aussi la découverte d'un fragment de plaque de marbre mouluré portant une partie de la dernière ligne d'une inscription : [EX] VOTO (Rémy, 1972). Enfin, le déblaiement des abords de la curie vers la même époque a livré deux importants fragments d'une mosaïque à décor géométrique noir et blanc, qui étaient situés à la cote 344,1 environ, à quelques mètres de l'angle extérieur sud-est du bâtiment (fig. 3; Stern, Blanchard-Lemée, 1975). Ces fragments, s'ils ćtaient en place, démontreraient l'existence d'une occupation gallo-romaine ancienne du secteur (compatible avec le type de la mosaique, datable de la première moitié du $\mathrm{I}^{\mathrm{er}} \mathrm{s}$. de notre ère), antérieure à l'implantation de cette partie du forum. En fait, tout porte à croire que ces fragments ne sont pas à leur emplacement d'origine : des débris conservés au musée de Feurs (la majeure partie des restes a été restaurée) montrent que la mosaique a été séparée de son radier à une date ancienne; de plus, le lieu de découverte de ces fragments correspond trop exactement au tracé du rempart médiéval pour qu'on ne pense pas qu'ils y aient été réemployés en fondation ${ }^{14}$.

La confirmation définitive de cette impression nous a été donnée par le sondage ouvert en 1988

14 I.e plan de situation publié par H. Stern et M. Blanchard-Lemée (pl. IIb) est localisable, contrairement à leurs dires, par comparaison avec le relevé de Gorce (notre fig. 3) : le massif de maçonnerie apparaissant au nord des fragments de mosaique correspond à l'angle sud-est de la curie: ces fragments sont donc alignés selon l'axe du rempart médiéval. dans la curie (fig. 9), qui montre que les sols de travail contemporains de la construction du bàtiment reposent immédiatement sur un niveau d'occupation protohistorique, sans aucune trace d'une occupation gallo-romaine intermédiaire. Les éléments datables livrés par cette couche d'occupation sont peu nombreux. Aucun ne parait postérieur au début du $\mathrm{I}^{\mathrm{rr}} \mathrm{s}$. après J.-C. :

- objets métalliques : un fragment de bracelet tubulaire en bronze, à décor de cannelures, objet typique de la phase dite La Tène [D1, qui s'achève avant le milieu du $\mathrm{I}^{\mathrm{r}}$ s. avant J.C. (Vaginay, Guichard, 1988, p. 152);

- céramique importée : un fragment de paroi de gobelet à décor moulé ("picots») de type Aco, datable de la première moitié de l'époque augustéenne, ainsi que 22 fragments d'amphore républicaine:

- céramique indigène tournée: un fond de vase peint à piédouche (Vaginay, Guichard, 1988, forme 1512-1614), datable de la période $50 / 20$ avant J.-C.. un fragment de paroi de bol à cuisson réductriceoxydante et surface extérieure fumigée décorée à la molette, datable de la seconde moitié du $\mathrm{I}^{\mathrm{er}} \mathrm{s}$. avant J.-C., deux lèvres de jattes à bord rentrant à cuisson réductrice-oxydante (formes 5111-5113), antérieures au milieu du $\mathrm{l}^{\mathrm{er}} \mathrm{s}$. avant J.-C., trois lèvres de vases ovoïdes à cuisson réductrice-oxydante, enfin un fragment de vase ovoïde de cuisson réductrice-oxydante, à col rainuré et micacé, forme confinée à Feurs dans les horizons augustéens (cf. infra, fig. 16, n 19);

- céramique indigène modelée: une lèvre d'écuelle profonde, appartenant à une phase ancienne du second $A g e$ du Fer, deux lèvres de jatte à bord rentrant, dont une à rebord épaissi. avec décor incisé (formes 5111 et 4113, antérieures au milieu du $\mathrm{I}^{\mathrm{rr}} \mathrm{s}$. avant $\mathrm{J} .-\mathrm{C}$.), une lèvre de vase ovoïde.

\section{LA PLACE CENTRALE ET LES PORTIQUES}

Cette partie du forum était restée inconnue jusqu'en 1978. Compte tenu des vestiges repérés antérieurement, autant à l'ouest (cryptoportiques) qu'à l'est (édifice de plan basilical) du terrain étudié, et de la simplicité de leur plan, l'identification des constructions dégagées lors d'une fouille de sauvetage en 1978-1979 ne pouvait faire de doute. Nous sommes ici en présence d'un alignement de cellules ${ }^{15}$

15 Le terme de "cellule" peut paraitre rague mais il nous semble préférable, parce que plus neutre, à celui de "boutique", couramment utilisé. Mème si toute activité commerciale - évoquée par ce terme - n est pas exclue sur un forum du Haut Fmpire, les fonctions économiques qu il abritait 


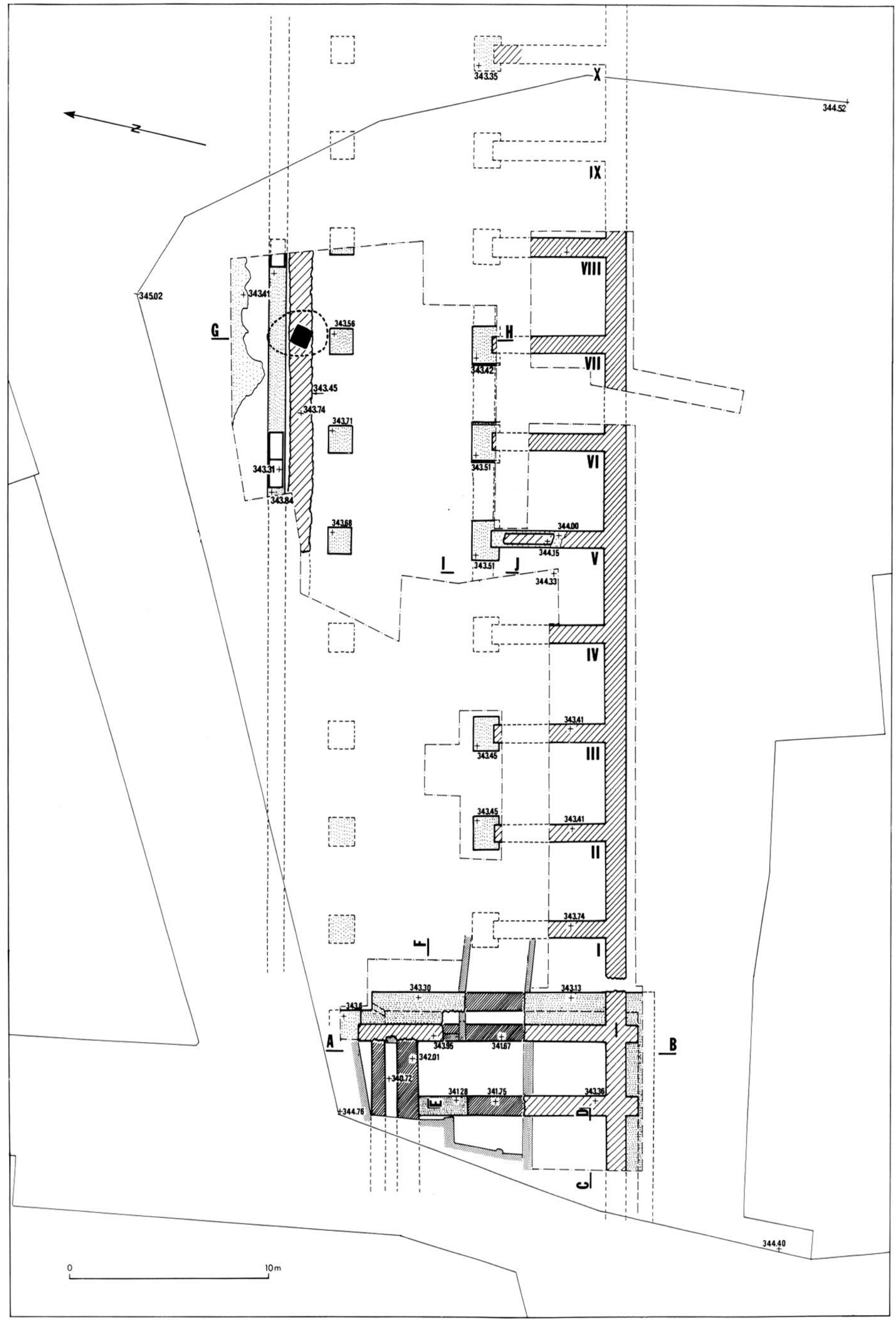

Fig. 12 - Plan des fouilles de l'aile sud des portiques de la place centrale, en 1978 (fig. 2, point 3); fouilles et relevés de P. Valette (les niveaux d'arasement des maçonneries sont signalés par les différents types de hachures). 
précédées d'un portique ouvert au nord sur la place centrale du complexe monumental, selon le schéma classique des fora tripartites, et se raccordant à l'ouest avec l'extrémité de l'aile sud du cryptoportique.

Les vestiges que recelait ce terrain, qui n'ont pu être que très partiellement étudiés, ont été détruits en 1981.

\section{Plan des cellules et du portique}

Huit cellules contiguës ont été dégagées, totalement ou en partie (fig. 12) (identifiées d'ouest en est : I à VIII). Des travaux ultérieurs de voirie nous ont permis de relever des maçonneries appartenant au prolongement vers l'est de cet alignement, portant à dix leur nombre minimum. Toutes s'appuient côté sud sur un mur de $1,05 \mathrm{~m}$ de largeur, conservé au maximum sur $0,6 \mathrm{~m}$ au-dessus de sa fondation, soit cinq arases, qui sont constituées d'appareil irrégulier de petit module. Les cellules sont séparées les unes des autres par des murs de refend nord-sud de $\overline{5}, 65 \pm 0,05 \mathrm{~m}$ de longueur, terminés vers le nord par une pile de fondation engagée de 1,7 sur 1,3 m appareillée sur deux assises à partir de son sommet (fig. 13). La cote de $1,7 \mathrm{~m}$ est celle relevée pour l'assise supérieure. Pour certaines piles (VI/VII, VII/VIII), la fondation est plus large de $0,3 \mathrm{~m}$ environ. Les murs ont une largeur de $0,9 \mathrm{~m}$ et sont appareillés de la même façon que le mur arrière. Cependant on observe sur celui qui est le mieux conservé (entre les cellules V et VI) un décrochement de l'appareil à $0,8 \mathrm{~m}$ au-dessus du bourrelet de fondation : au-delà, l'élévation, conservée sur une assise seulement, n'est large que de $0.6 \mathrm{~m}$ et construite en petit appareil régulier. Si l'on tient compte de ces décrochements dans les murs latéraux, la largeur dans l'œuvre des cellules est de $3,9 \mathrm{~m}$ (avec des variations de moins de $\mathbf{5} \mathrm{cm}$ de l'une à l'autre). Leur superficie serait donc voisine de $22 \mathrm{~m}^{2}$.

L'emplacement de la colonnade du portique ouvert sur la place est signalé par des piles de 1,3 sur $1,3 \mathrm{~m}$ situées dans l'alignement des murs de séparation des boutiques, et distantes de $6,0 \mathrm{~m}$ des piles engagées qui les précèdent. Comme ces dernières, elles présentent deux assises appareillées au-dessus du sommet de leur fondation.

Le mode de construction des maçonneries paraît dans l'ensemble très homogène : la seule pierre utilisée est le granite rose, les fondations sont constituees d'un blocage jeté en tranchée, leur sommet est sou-

étaient en effet essentiellement "officielles" (cf. par exemple : Le Gall, 1978; Andreau, 1987).

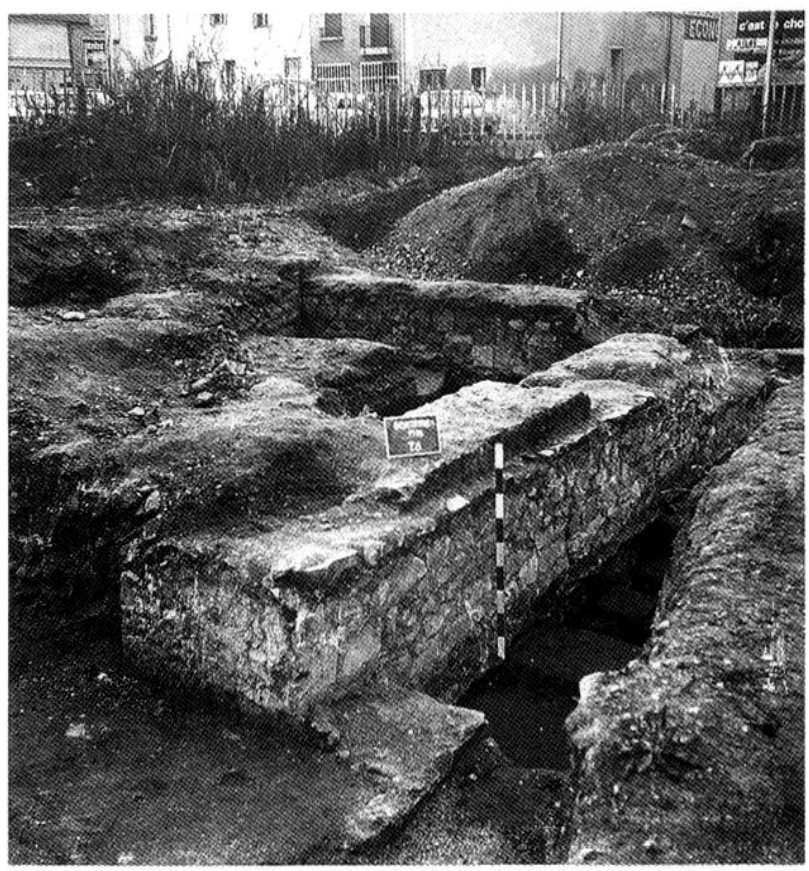

Fig. 13 - Mur de séparation des cellules V et VI de l'aile sud des portiques de la place centrale, avec, au premier plan, une pile de fondation engagée, au second plan la fondation appareillée du mur supportant la base de l'élévation (1978)

ligné par un fort bourrelet de mortier. Toutes sont puissamment ancrées dans le sol puisque les fondations sont profondes de 1,8 à $2 \mathrm{~m}$ pour les murs des cellules (piles engagées incluses), et $2,4 \mathrm{~m}$ pour les piles du portique. Le parement de petit appareil irrégulier est parfaitement identique pour l'ensemble des murs. Quelques maladresses de construction, qui contrastent avec la qualité générale des maçonneries, laissent cependant possible l'existence de reprises: l'appareillage de l'assise supérieure des piles de la travée nord du portique est nettement moins soignée que l'assise inférieure (moellons irréguliers, remploi de calcaire jaune, jointoyage négligé).

\section{Nivellement}

Si le plan d'ensemble des constructions dégagées ne présente pas de difficultés d'interprétation, il n'en va pas de même pour la compréhension de la stratigraphie et la restitution des niveaux de circulation antiques, ce qui s'explique par les perturbations provoquées par une occupation intense du sol pendant.le Moyen Age et l'époque moderne. Les conditions matérielles précaires dans lesquelles se sont déroulées ces fouilles n'ont fait qu'aggraver cet état de fait, puisqu'on a dû se résoudre à programmer les campagnes au coup par coup, ce dont témoigne le découpage plutôt anarchique des zones fouillées, et à sacri- 


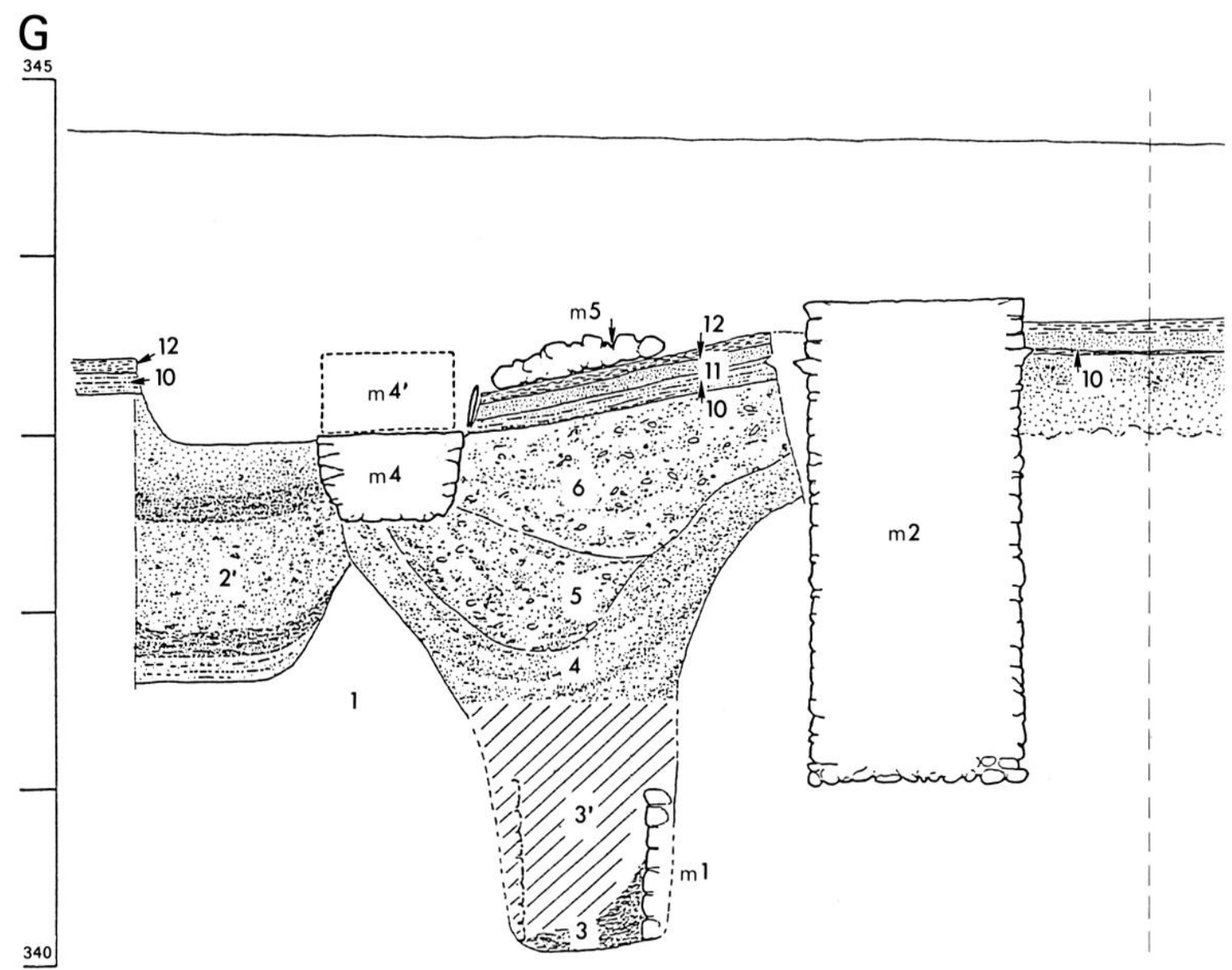

fier les niveaux d'occupation situés au-dessus des premiers horizons antiques bien constitués.

Le sommet des fondations maçonnées se situe au niveau du sol primitif ou légèrement plus haut. Celui-ci accuse une légère pente d'est en ouest : il est à la cote 343,40 dans la cellule VIII et seulement à 343,04 dans la cellule III. Dans les cellules, il a donc servi de niveau de travail pour la construction des murs mais l'absence totale de vestiges gallo-romains et d'aménagement de sol exclut qu'il ait pu servir de niveau de circulation durant la période d'utilisation normale du monument. De fait, il est surmonté par une couche stérile de gravier, conservée dans le meilleur des cas jusqu'à la cote 343,7 (dans la cellule III) (fig. 14, couche 8), qui peut fort bien être considérée comme un remblai lié à la construction.

La stratigraphie de la zone couverte du portique est plus complexe. On remarque d'abord que les cellules et le portique sont séparés par une tranchée peu profonde de profil en $\mathrm{U}$ régulier (largeur : $0,9 \mathrm{~m}$, profondeur : $0,4 \mathrm{~m}$ ) creusée à partir du sol primitif et remplie par un remblai homogène de sable (fig. 14, couche 7). Sa paroi nord est alignée sur le parement des piles engagées. Si sa relation avec la construction du monument est certaine, son profil empèche d'y voir les vestiges d'un état antérieur du forum comme une empreinte de fondation de maçonnerie disparue; on peut proposer qu'il s'agit d'une tranchée d'alignement, ouverte lors du creusement des fondations des cellules et destinée à régler l'axe du portique. Sur l'ensemble de la surface du portique, le sol primitif est recouvert par un épandage de mortier blanc, très diffus par endroits, nettement plus conséquent à d'autres (au-delà de l'alignement des piliers vers le nord - cf. infra), situé à la cote $343,40 \pm 0,05$ (fig. 14, couche 10). Il est recouvert par un remblai (couche 11) qui a noyé des épandages d'éclats de granite rose et de galets (couche 11'), vestiges d'aires de travail liées à la construction. Ce remblai est luimême scellé par une couche lassée constituée de débris de calcaire jaune, ćpaisse de quelques centimètres (couche 12). Son sommel, qui est horizontal dans le périmètre du portique (cote : $343,60 \pm 0,05$ ), noie la première assise appareillée des piles de la travée nord (le sommet de ces piles est à la cote 343,70 $\pm 0,05)$ et domine légèrement le sommet des piles engagées (situé à la cote $343,48 \pm 0,03$ ). Il n'a pas èté possible d'observer de couche archéologique antique en place au-delà du sommet de ce sol de calcaire jaune broyé. Il est en fait exclu de pouvoir le considérer comme un niveau de circulation définitif du 


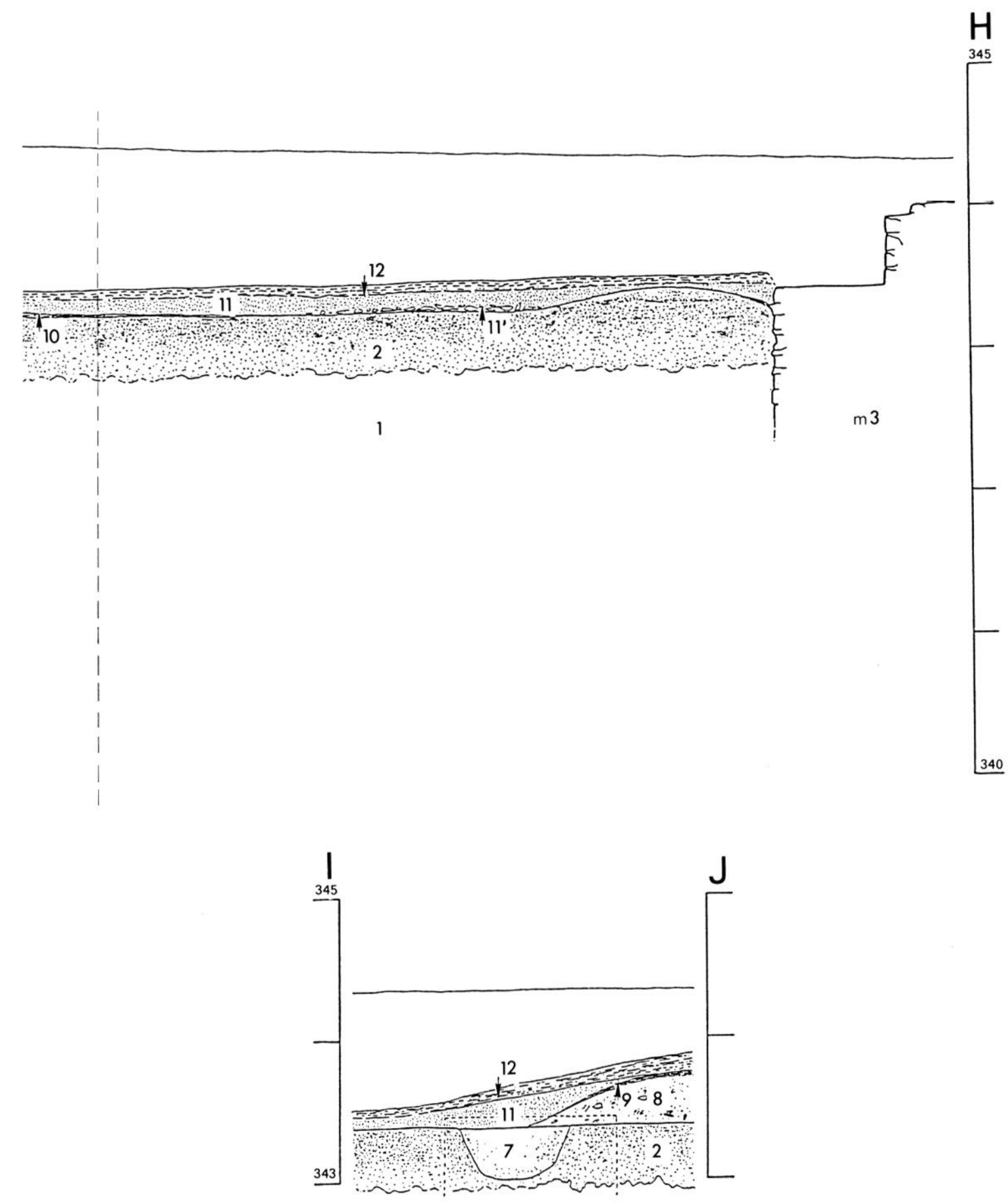

Fig. 14 - Coupes du portique selon G-HI et I-J (localisées sur la fig. 12).

Maçonneries : $\mathrm{ml}$ : parement du puits gallo-romain antérieur au forum; $\mathrm{m} 2$ : pile de fondation du portique; $\mathrm{m} 3$ : pile de fondation engagée dans le mur de séparation des cellules VII et VIII $m 4$ : massif de fondation parallèle au portique; $\mathrm{m}^{\prime}$ : bloc de grand appareil (emplacement) posé sur la fondation $\mathrm{m} 4 ; \mathrm{m} 5$ : massif de maçonnerie grossière parallèle au portique.

Couches de terrain : 1 : sol naturel ; 2 : terre brune gravillonneuse (niveau d'occupation gaulois) ; $2^{\prime}:$ fosse s'ouvrant dans la couche 2 ; $3:$ terre limoneuse riche en mobilier (comblement primaire du puits) $: 3^{\prime}:$ partie détruite $; 4:$ terre brune sans vestiges $: 5:$ gravier mêlé de blocs de terre brune, sans vestiges; 6 : gravier grossier, sans vestiges $(4,5$ et 6 sont des remblais de comblement du puits) $; 7$ : sable (remplissage d'une tranchée alignée selon l'axe du portique, antérieure à sa construction); $8:$ gravier grossier (remblai liè à la construction des cellules); 9 : couche charbonneuse : 10 : couche de mortier blanc feuilletée (sol de travail lié à la construction du portique); 11 : terre grise sableuse (remblai lié à la construction du portique); $11^{\prime}$ : amas d'éclats de taille de granite rose à la base de 11: 12 : épandage de calcaire jaune concassé (sol de travail). 
portique, à cause de son irrégularité (on remarque en particulier qu'il remonte en plan incliné à la hauteur de l'ouverture des cellules sur le portique). On a vu qu'un sol de même nature, retrouvé dans la basilique, peut en toute vraisemblance avoir servi de radier à un dallage. Plusieurs autres indices convergents permettent ici de restituer le niveau de circulation des cellules et du portique, que l'on peut supposer de plain-pied, à un niveau sensiblement plus élevé. C'est d'abord le décrochement de l'appareil des murs latéraux des cellules, à la cote 344,0 (conservé sur celui séparant les cellules V et VI), qui correspond à l'amorce d'une élévation beaucoup plus soignée que la partie inférieure du mur (on remarquera d'ailleurs que, dans le cas de la curie, les parements utilisant des moellons irréguliers, comme ici la base des murs, étaient destinés à être remblayés). On doit également supposer que les piles maçonnées servaient de support à des blocs de grand appareil affleurant le niveau de circulation, qui recevaient à leur tour la base des colonnes du portique, comme on a pu l'observer dans la basilique. En fait, une trace d'arrachement de bloc de grand appareil est nettement discernable sur la pile engagée liée au mur de séparation des cellules V et VI : elle paraît correspondre à un bloc rectangulaire étroit de 0,8 sur $1,6 \mathrm{~m}$. Si l'on restitue une hauteur de 0,45 à $0,5 \mathrm{~m}$ pour ces blocs, conforme aux dimensions de ceux conservés (deux dans la basilique, trois dans l'emmarchement au nord du portique - cf. infra), on atteint une altitude de 343,9 à $344,0 \mathrm{~m}$, cohérente avec celle estimée auparavant par un autre biais. On adoptera donc la cote de 344,0 comme estimation du niveau de circulation du portique et des cellules.

Le niveau de circulation extérieur au forum, audelà du mur d'appui des cellules, n'est pas restituable précisément puisque les quelques sondages effectués au sud de ce mur n'ont pas permis de mettre en évidence de sol antique autre que le sol primitif.

\section{Liaison du portique et de la place}

Au-delà de la travée de piles du portique, l'épandage de mortier blanc reposant sur le sol primitif devient plus épais. Sur la longueur dégagée du portique (une dizaine de mètres), il est recoupé par une tranchée de contour assez régulier au sud, beaucoup plus sinueux au nord, qui a servi à installer la fondation d'un massif de maçonnerie. Ce massif supporte un alignement de blocs de grand appareil (fig. 14, massif $\mathrm{m} 4$ ). Ces blocs de calcaire jaune, (dimensions : $0,8 \times 1,3 \times 0,45 \mathrm{~m}$ ), dont trois ont été retrouvés en place, sont distants de $2,2 \mathrm{~m}$ des piles du portique; leur sommet, situé à la cote 343,30 $\pm 0,03$, est au niveau du sol de mortier blanc. Ia fondation tranche nettement sur les autres maçonneries par son irrégularité et sa faiblesse : large de $0,9 \mathrm{~m}$, elle n'est profonde que de $0,7 \mathrm{~m}$ et son blocage contient de nombreux éclats de calcaire jaune. Le comblement de la tranchée, qui semble avoir été perturbé ultérieurement (sans doute lors de la récupération des blocs de grand appareil), puisque de la céramique médiévale y a été trouvée, contenait d'assez nombreux fragments de ce même calcaire, dont l'essentiel des éléments d'architecture retrouvés sur la fouille. Le sol de calcaire jaune concassé retrouvé à l'emplacement du portique est également interrompu par la tranchée de fondation; il disparaît audelà. Un autre massif de maçonnerie de même orientation occupait l'espace situé entre les piles du portique et l'alignement de blocs de grand appareil, reposant directement sur le sol de calcaire jaune concassé (fig. 14, massif m5). Sa facture est très grossière : il s'agit d'un blocage non parementé, irrégulièrement arasé (cote maximale du sommet : 343,75 ), large de $1,1 \mathrm{~m}$ environ, constitué de matériaux divers : granite rose, calcaire jaune (dont des fragments d'architecture), barytine.

L'interprétation de ces aménagements demeure incertaine. Ils sont situés à un emplacement où l'on s'attend à trouver un emmarchement permettant d'accéder de la place centrale du forum au portique: d'autre part, leur facture grossière et leur position stratigraphique incitent à les attribuer à des travaux postérieurs au programme initial (desquels pourrait également procéder une réfection du portique, en relation avec l'exhaussement d'une assise des piles de fondation). Il n'en reste pas moins que leur alignement et la nature du mortier qu'ils utilisent les désignent certainement comme antiques, et permettent effectivement de considérer leur intégration à la structure d'un emmarchement comme plausible, même si celui-ci n'est pas lié au programme primitif.

Le niveau de circulation de la place ne peut d'ailleurs pas être restitué précisément : il est en tout cas situé à une cote supérieure à celle de l'épandage de mortier qui recouvre le sol primitif, mais il est plausible de restituer un caniveau de drainage à la base de l'emmarchement, qui dirigerait les eaux de ruissellement du portique vers le regard dégagé plus à l'ouest, à l'angle de la galerie à cryptoportique, et qui se déverse dans le conduit d'assainissement inclus dans ses murs (cf. infra). En fait, deux fragments de rigoles monolithes en granite, d'un type identique à celui en usage le long des portiques de rues, ainsi qu'il en a été dégagé par exemple en 1984 , 
rue Saint-Antoine, et qui ont été retrouvés dans le comblement de la tranchée de fondation de l'alignement de blocs de grand appareil, pourraient attester leur existence. Avec une épaisseur de $0,3 \mathrm{~m}$ environ, l'utilisation de ces rigoles obligerait à situer le niveau de la place au-dessus de la cote 343,70 . On conservera donc comme estimation de la hauteur de l'emmarchement entre place et portique la fourchette comprise entre 0,3 et $0,7 \mathrm{~m}$.

\section{Liaison avec le cryptoportique}

La rangée de cellules dégagée vient buter à l'ouest contre l'extrémité de la branche sud de la galerie à cryptoportiques qui circonscrit le temple (cf. infra). La largeur de la première cellule (I), accolée au cryptoportique, est supérieure à la normale de $0,3 \mathrm{~m}$, soit une largeur dans l'œuvre de $4,0 \mathrm{~m}$, mesurée à la base de l'élévation. Le long mur est-ouest auquel sont adossées les cellules se raccorde à un mur de largeur sensiblement identique intégré à l'élévation du cryptoportique. Bien que leur mode de construction semble parfaitement homogène (mortier notamment), le raccord entre les deux murs se manifeste par un décrochement du parement de quelques centimètres sur le côté nord et par le passage à un appareillage en petit moellons très réguliers sur le côté sud. Il est donc vraisemblable que le mur sur lequel s'adossent les cellules a été construit après l'ensemble du cryptoportique, tout en s'intégrant au même programme architectural. Dans le prolongement de la travée nord du portique et dans l'axe de la paroi ouest de la cellule I, on a pu dégager partiellement un massif de maçonnerie dont le niveau supérieur coïncide exactement avec celui des piles. Ce massif, qui était recouvert d'importants fragments de calcaire jaune délité, vestiges probables d'un bloc de grand appareil, est limité vers le sud, dans l'alignement du parement sud des piles, par un contrefort appuyé contre le mur du cryptoportique, saillant de $1,95 \mathrm{~m}$; cette pile solidaire de l'angle du cryptoportique permettait d'arrêter le portique par une colonne engagée dans ce contrefort (fig. 15). Des observations ultérieures sur l'aile nord du forum (printemps 1991) ont montré que cette pile ne se prolongeait que de $1,10 \mathrm{~m}$ au-delà du contrefort en direction de l'axe du forum. Le sol environnant (sol de travail) était constitué de trois radiers superposés contenant un blocage hétérogène (granite rose, tuiles, éclats de calcaire jaune) lié par un mortier maigre. Son sommet était, à la cote 343,6 , identique à celle des portiques.

Un regard de 0,2 sur $0,2 \mathrm{~m}$ ménagé dans l'angle nord-est du cryptoportique, dont le sommet est situé

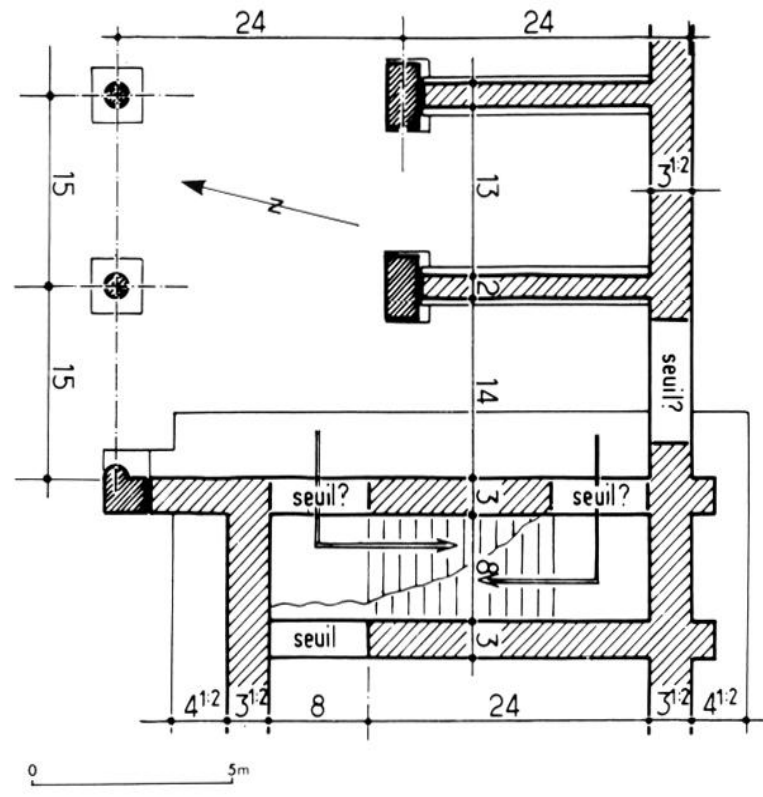

Fig. 15 - Restitulion de la liaison du portique et de la galerie à cryptoportiques (avec dimensions portées en pieds).

à la cote 342,80 , permettait vraisemblablement d'évacuer les eaux de ruissellement de la place centrale. Un deuxième regard était ménagé contre la paroi ouest du contrefort (observations de 1991). Son entrée était obstruée par un blocage aéré contenant des pierres de nature diverse, lié par un mortier maigre.

\section{Données stratigraphiques}

Les sols de travail et remblais antiques liés à la construction du portique n'ont pas livré le moindre mobilier archéologique. En revanche, les niveaux d'occupation situés sous ce que l'on a dénommé sol primitif, et qui correspond à l'état du terrain tel que l'ont trouvé les constructeurs du monument, ont livré un abondant mobilier des II $^{\mathbf{e}}$ et $\mathrm{I}^{\mathrm{er}}$ s. avant $\mathbf{J}$.-C. Malheureusement, la phase la plus récente de cette occupation, durant la seconde moitié du $\mathrm{I}^{\text {er }} \mathrm{s}$., est aussi la plus diffuse, ce que l'on a mis au compte d'un déplacement de l'habitat gaulois. Les éléments les plus récents (Vaginay, Guichard, 1988, fig. 10 et p. 24) sont certainement situables après le début du principat, sans que l'on puisse vraiment préciser davantage : quelques infimes fragments de gobelets ovoïdes à parois fines, de sigillée italique et de bols peints de type Roanne anciens, un ardillon de fibule à charnière.

Néanmoins, le mobilier provenant d'un puits remblayé lors de la construction du portique fournit des indices de datation d'une certaine précision. Ce puits a été retrouvé à proximité de la pile de la tra- 
vée nord située dans l'axe de la séparation entre les cellules VII et VIII. Bien qu'ayant déjà été fourni dans une publication antérieure (Valette, 1981), l'inventaire des objets qui ont été recueillis mérite donc d'être rappelé.

Une tranchée de sondage pratiquée au nord de la pile en question avail d'abord permis de mettre en évidence, sous le sol de mortier blanc déjà décrit, affaissé et fort épais à cet emplacement, une fosse de $3 \mathrm{~m}$ de diamètre environ, remplie d'une série de couches de remblais à peu près stériles, sur une épaisseur de plus de $2 \mathrm{~m}$, recoupées par la fondation de la pile (fig. 14, couches $4,5,6$ ). La fouille n'avait pu être menée jusqu'à la base de ce comblement en raison des dangers d'éboulement; c'est à l'occasion d'un sondage géologique pratiqué ensuite au même emplacement qu'on a pu découvrir l'appareillage du puits. Sa présence même à cet endroit paraît insolite, en raison de l'absence totale de vestiges d'habitat gallo-romain alentour, vérifiée par la fouille méthodique de plus de $100 \mathrm{~m}^{2}$ des niveaux d'occupation antérieurs à l'installation du forum; son mode de construction : un appareillage en blocs de granite gris de module moyen suivant un plan carré de 0,8 sur $0,8 \mathrm{~m}$, est une étrangeté supplémentaire qui permet de douter de son utilisation à des fins purement domestiques, malgré la banalité du mobilier de son remplissage, à moins que ce plan ne s'explique par le prolongement de traditions gauloises, puisque à La Tène finale les coffrages de puits carrés (mais en bois) sont la règle générale. Autre constatation, celle-là essentielle du point de vue de la datation : le parement du puits avait été démonté sur plus de $2 \mathrm{~m}$ avant son remblayement. Cette opération peut être mise en rapport avec le nivellement du terrain préalable à l'installation du portique. On en déduit que le puits est restè ouvert (pour que son parement puisse être démonté) jusqu'à la mise en place du portique et que le mobilier issu de son remplissage doit fournir un lerminus posl quem très peu éloigné dans le temps de la construction de ce dernier. Fort heureusement, l'échantillonnage d'objets est suffisant pour permettre de cerner avec une certaine précision la date de son comblement.

Le remplissage contenait un as d'Auguste frappé à Rome en 23 avant J.-C. (Cohen 503), et deux fibules en bronze à ressort protégé (fig. 16, $\mathrm{n}^{\text {os }} 1$ et 2), l'une du type dit à arc continu (Feugère, 1985, type 14b; Guillaumet, 1985, type 12101), bien datable de l'époque augustéenne (ce que confirme l'examen des contextes de découverte régionaux, ainsi la présence d'un exemplaire analogue dans la tombe 16 de la nécropole gauloise tardive de Feurs, datable de 25-15 avant J.-C.), l'autre du type dit à disque médian (Feugère, 1985, type 15a ; Guillaumet, 1985, type 11303), qui paraît avoir été introduit en Gaule dès le milieu du $\mathrm{I}^{\mathrm{rr}} \mathrm{s}$. avant J.-C., mais ne fut largement diffusé qu'au cours du règne d'Auguste.

La céramique peut être divisée en deux lots. Le premier correspond à des types de vases représentés par des exemplaires peu fragmentés et donc directement liés à l'utilisation du puits, le second à des types représentés seulement par de petits tessons et donc assimilables à du mobilier résiduel.

Le premier lot comprend quatre types de vases :

- des cruches à ouverture large tronconique, munies d'une anse et présentant un décor sur plusieurs registres réalisé au lissoir ou au peigne. Cinq individus au moins sont représentés, dont trois à cuisson réductrice (B) (fig. $16, \mathrm{n}^{\text {os }} 3$ à 5 ) et trois à cuisson réductrice-oxydante (A) (n ${ }^{\text {os }} 6$ à 8). En Forez, cette forme est typique des horizons gallo-romains anciens. Elle apparaît à Roanne dans des contextes datables du milieu du règne d'Auguste (Poncet et alii, 1979, pl. XI no 8). A Feurs, elle est présente en particulier dans une couche scellant la construction de l'égout du forum (couche VII), datée autour de 20 après J.-C. (Vaginay, Valette, 1982, fig. $15 \mathrm{n}^{\circ} 12$ et infra) et dans plusieurs fosses-dépotoirs découvertes sur le site; son utilisation ne parait pas se prolonger au-delà de la fin du règne de Tibère. Dans la région lyonnaise, l'apparition du type est également située avant 10 avant $J$.-C., mais il se maintiendrait durant tout le $1^{\text {er }}$ s. après J.-C. (Desbat, Mérigoux, 1979, pl. $\left.4 n^{\circ} 6\right)$;

- des flacons globulaires ansés à goulot étroit et pâte calcaire, claire et fine, représentés par trois exemplaires, l'un presque complet (fig. 16, $n^{\circ} 9$ ), les deux autres très fragmentés (dont un est à engobe rouge externe). Cette forme n'est pas datable précisément : si elle ne devient très fréquente qu'à partir du règne de Tibère, on trouve à Feurs des exemplaires importés datables du tout début du règne d'Auguste (nécropole gauloise tardive);

- des vases ovoïdes de volume assez faible, représentés par cinq individus, dont un complet. Quatre présentent des caractéristiques identiques : cuisson réductrice, pied annulaire, décor de bandes lissées (fig. 16, $n^{\text {os }} 10$ à 13 ); le cinquième ( $\left.n^{\circ} 14\right)$ se distingue par la forme de sa base et surtout par des parois plus minces et une pâte cuite à réduction très sonore. Cette forme de récipient apparaît à Feurs dans des contextes datables du début du règne d'Auguste (nécropole gauloise) et ne paraît plus utilisée dès la fin du règne de cet empereur;

- un gobelet cylindrique (fig. $16, n^{\circ} 15$ ), repré- 

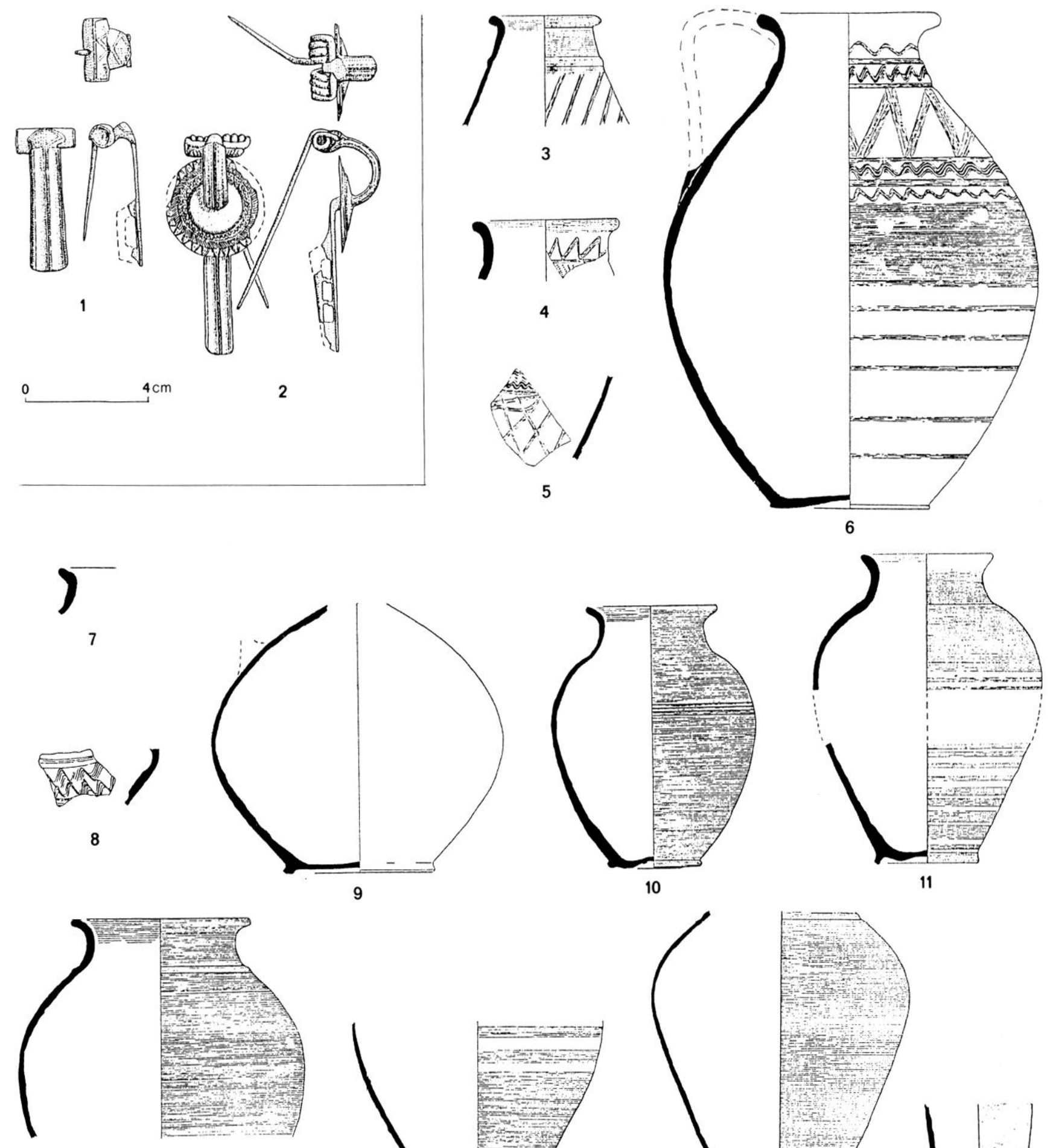

12
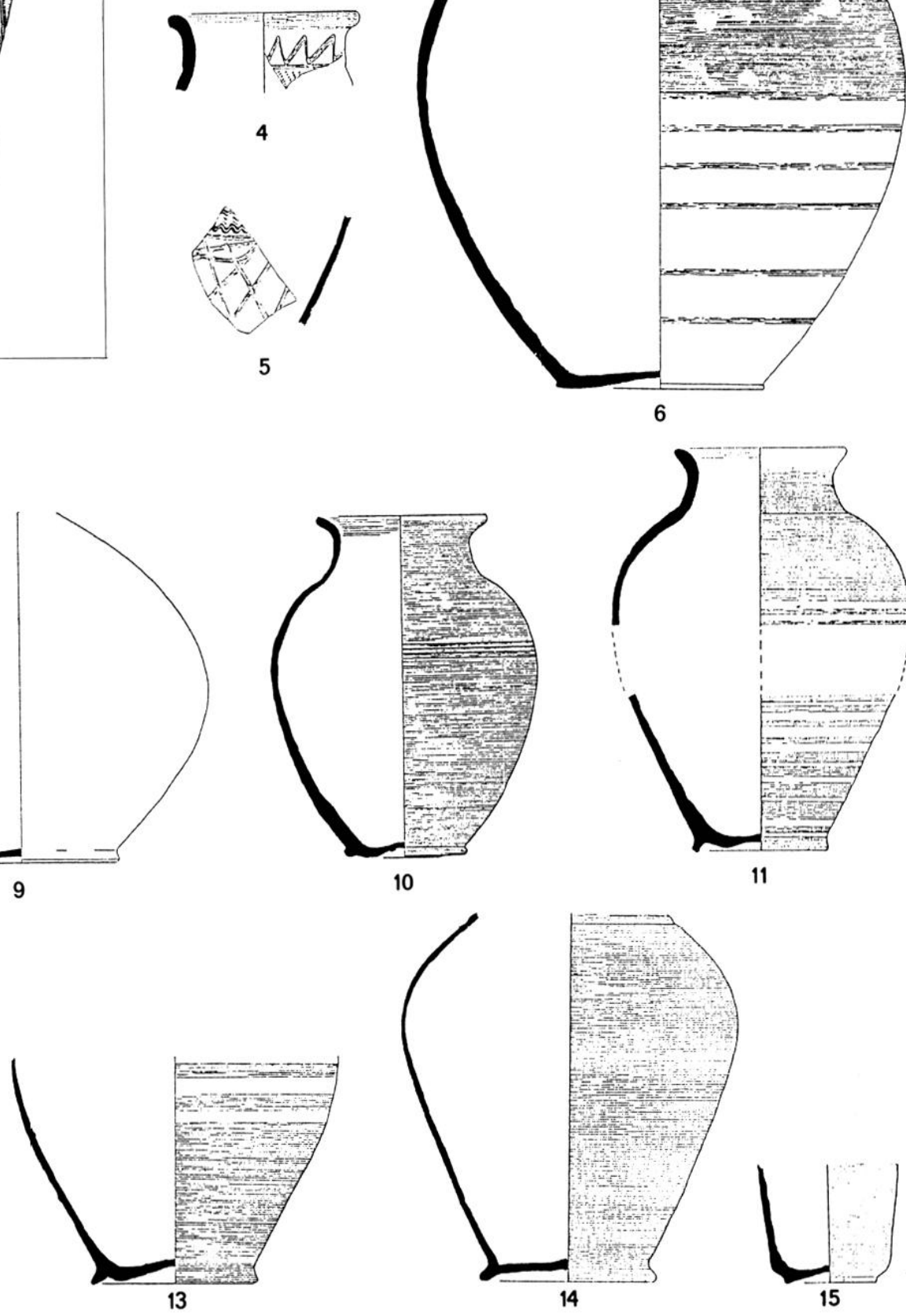

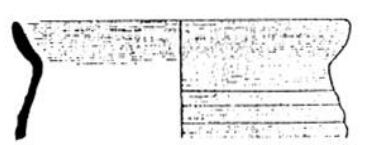

16

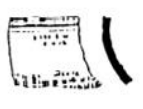

17

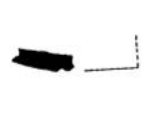

18

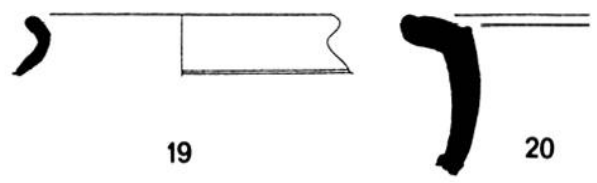

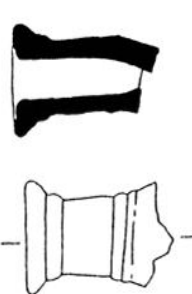

21

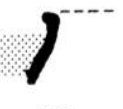

22

Fig. 16 - Mobilier du puits gallo-romain recouvert par les niveaux de construction du forum (céramique : èch. 1/4, fibules : éch. 1/2); le puits est situé à proximité de la pile de fondation du portique située dans l'axe du mur de séparation des cellules VII et VIII (cf. fig. 12). 
senté par un gros fragment, de fabrication apparemment locale. Ce récipient ne possède pas d'analogue dans le répertoire régional de la céramique domestique. En revanche, il est plausible de le considérer comme une imitation de gobelet cylindrique à parois minces d'un type datable du milieu de la période augustéenne.

Le second lot comprend :

- une jatte à rebord en gouttière, de cuisson réductrice (fig. 16, $\mathrm{n}^{\circ} 16$ ), type très fréquent en Forez et datable dans le dernier tiers du ${ }^{\text {er }} \mathrm{s}$. avant J.-C. (par exemple: Poncet et alii, 1979, pl. X $\left.n^{\text {os }} 1-2\right)$;

- des jattes apodes à décor à la molette, représentées par un fragment de paroi et un fragment de fond ( ${ }^{\text {os }} 17$ et 18 ), datables dans le même intervalle;

- un fragment de paroi de patère à vernis orangé, vraisemblablement identifiable à une production de la région lyonnaisc, cncore datable dans le même intervalle;

- un rebord de vase ovoïde à ouverture large, col court rentrant et couverte micacée, orné de rainures à la liaison col-panse (fig. 16, no 19). A Feurs, ce type de production paraît caractéristique des horizons du début de notre ère ${ }^{16}$;

- un rebord de grand vase de stockage (fig. 16, $\mathrm{n}^{0} 20$ ), dont des exemplaires analogues se retrouvent dans la couche VII, au-dessus de l'égout du forum (Vaginay, Valette, 1982, fig. 15, nos 13-14). Ce type apparait certainement avant la fin du règne d'Auguste (Poncet el alii, 1979, pl. XI n 2);

- un manche de poêlon (?) en céramique à cuisson réductrice-oxydante et couverte micacée ( $\left.n^{\circ} 21\right)$, sans comparaison régionale ;

- un rebord de jatte peinte de forme 3221 (fig. 16, no 22), dont la production ne dépasse pas le milieu du règne d'Auguste (Vaginay, Guichard, 1988, fig. 61).

Il faut enfin ajouter à ce lot une cinquantaine de fragments d'amphores républicaines et une série de tessons gaulois plus anciens que ceux décrits cidessus.

A l'examen de ce catalogue, il nous semble aujourd'hui plus juste d'élargir l'intervalle chronologique qui avait été initialement proposé pour ce mobilier (le premier quart du $\mathrm{r}^{\mathrm{er}} \mathrm{s}$. après J.-C., dans : Valette, 1981) à la fin du siècle précédent. On retiendra surtout que le comblement du puits fut probablement achevé avant la fin du règne d'Auguste.

16 Forme associée par exemple à un demi-as de Nìmes, de la sigillée italique, une tasse en céramique de cuisson réductrice de forme Goudineau 13 dans une fosse fouillée en 1987.

\section{LA GALERIE À CRYPTOPORTIQUES ET L'AIRE DU TEMPLE}

Les vestiges visibles de la galerie à cryptoportiques, et tout particulièrement le conduit d'assainissement inclus dans son mur périphérique, qui est accessible à partir de plusieurs caves modernes, ont depuis longtemps excité la curiosité des Foréziens. Un premier plan d'ensemble fut relevé avant 1850 par Roux (fig. 17), lequel a tout de suite établi un lien entre ces substructions et le forum de la ville antique. Il en fait la description suivante (Roux, 1851 , p. 43-44) : "Ces souterrains forment un système d'égouts ou cloaques qui se développent sur deux lignes, suivant le plan d'un immense parallélogramme dont ils occupent trois côtés. La façade principale a quatre-vingts mètres d'étendue; les deux autres en ont soixante. La superficie qu elles circonscrivent est de quatre mille huit cent mètres carrés. Ces deux rangs laissent entre eux un intervalle de seize mètres, qui se trouve réduit à douze, vers l'extrémité des deux côtés, par un retrait symétrique de quatre mètres dans les parties nord et sud. L'égout a sous clef un mètre quatre-vingt-cinq centimètres de hauteur, et soixante centimètres de largeur. Il est construit en petit appareil allongé, lié par un ciment grisâtre et très dur. On a établi dans l'épaisseur des parois, des larmiers [sic; comprendre : des regards] placés de distance en distance sur un plan incliné de haut en bas [...]. Près de l'angle nord, du côté droit, on remarque une ouverture d'un mètre, dont le cintre est fait de grandes tuiles à rebord alternant avec des claveaux de pierre. A l'angle nord-ouest, la ligne de face ouest et la ligne latérale nord se réunissent en un seul grand égout qui traverse la route de Roanne et se dirige vers le jardin de l'hôpital [...]. Nous sommes forcément conduit à voir dans ces souterrains, les cloaques qui assainissaient les promenades d'un forum, tandis que leur

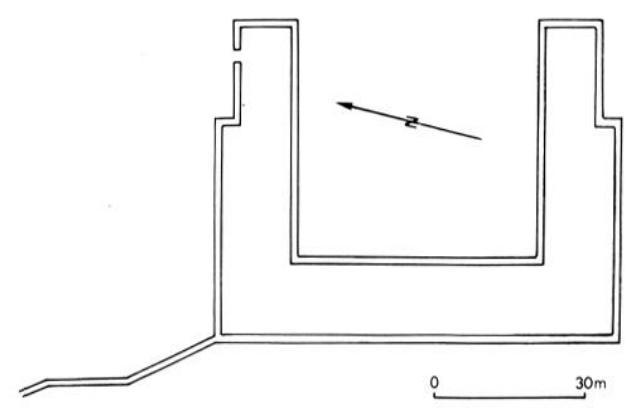

Fig. 17 - Plan du conduit d'assainissement de la galerie à cryptoportiques d'après les relevés de Roux (1851). 
maçonnerie servait de fondation à ses portiques ". On ne sait cependant rien de la méthode qu'il a suivie pour effectuer ses relevés, ni même la localisation précise des vestiges qu'il a pu observer. De plus, le plan qu'il fournit est très sommaire. Une nouvelle campagne de relevés systématiques des vestiges s'imposait donc.

Une fouille de sauvetage plus importante a également permis de dégager l'extrémité est de la branche sud du cryptoportique en 1978 à l'occasion de réaménagements urbains (fig. 12). On insistera donc particulièrement sur les résultats de ce chantier, avant de fournir les données complémentaires sur cette partie du monument issues de la prospection des caves des constructions actuelles du quartier, d'un sondage limité dans l'angle intérieur sudouest du cryptoportique et des observations effectuées à l'occasion de travaux de voirie au printemps 1991. On a consigné en annexe l'inventaire des caves qui ont pu être visitées (cf. infra, p. 161).

\section{L'extrémité est de l'aile sud du cryptoportique}

\section{Plan}

C'est le tracé du conduit d'assainissement percé à la base des murs du cryptoportique qui avait permis à Roux d'identifier le plan de l'ensemble. Conformément à ses indications, on a pu constater que ce conduit est un couloir voûté, haut à l'origine de $1,95 \mathrm{~m}$ et large de $0,6 \mathrm{~m}$ (fig. 18, 19). L'appareil des parois latérales présente un aspect plus ou moins régulier tant dans la taille des moellons que dans leur mise en place. La voûte, épaisse d'une soixantaine de centimètres, est montée sur un coffrage dont les planches de couchis, toujours au nombre de 6 et larges de 0,16 à $0,19 \mathrm{~m}$, ont laissé leur empreinte dans le mortier. Les pierres de son blocage sont sommairement agencées à la manière de claveaux.

Le plan de la partie fouillée demeure clairement lisible malgré les perturbations postérieures (fig. 12). C'est donc le tracé du conduit qui est l'élément essentiel pour la compréhension de l'ensemble. Il affecte la forme d'un $\mathbf{U}$ ouvert vers l'ouest, dont seule la branche nord-sud a été reconnue dans sa totalité. Une cave avait réoccupé une portion de l'espace circonscrit par ce conduit, en débordant au-delà vers le nord. Une partie des murs antiques était donc conservée sur une hauteur importante, tandis que du côté nord et à l'emplacement de la descente de la cave ils étaient arasés à environ $1,0 \mathrm{~m}$ au-dessous du niveau de l'intrados de la voûte du conduit. Le mur dédoublé pour recevoir le conduit d'assainissement a une largeur totale de $2,4 \mathrm{~m}$, qui est distribuée entre deux maçonneries de $0,9 \mathrm{~m}$ de part et d'autre du

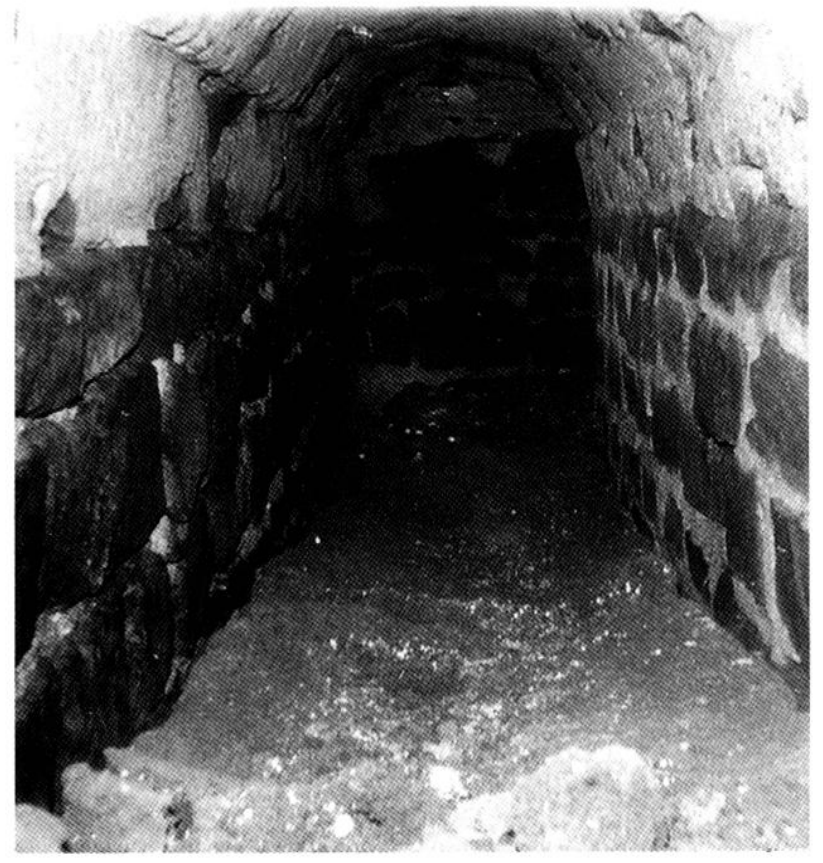

Fig. 18 - Vue de l'intérieur du conduit d'assainissement. situé à la base des murs du cryptoportique (extrémité de l'aile sud; 1978).

vide pour le segment dégagé orienté nord-sud, et entre une maçonnerie de $1,05 \mathrm{~m}$ sur le côté interne du cryptoportique, et une autre de $0,75 \mathrm{~m}$ sur le côté externe pour les parties orientées ouest-est. Dans l'angle nord-est, ce mur est percé d'un regard horizontal étroit $(0,2 \times 0,2 \mathrm{~m})$ au niveau de la base de la voûte du conduit, qui débouche à l'extérieur de la galerie.

Ce mur délimite donc un niveau de circulation semi-enterré de largeur dans œuvre de 9,4 m, qui n'a été dégagé que sur une longueur de $6 \mathrm{~m}$ à cause de la proximité d'une rue moderne, et dont l'élévation conservée depuis le sommet des fondations atteint encore 2,9 m par endroits. La largeur de la galerie coïncide précisément avec la cote de $12 \mathrm{~m}$ fournie par Roux pour l'écartement entraxes des deux lignes de conduit aux extrémités des branches latérales. Le mur périphérique n'était élargi qu'à sa base afin de ménager l'emplacement du conduit. Son sommet forme une plate-forme sur laquelle s'appuie l'élévation proprement dite qui est constituée de murs nettement plus minces : $0,9 \mathrm{~m}$ pour le segment nordsud, d'aplomb avec la façade interne du cryptoportique, $1,05 \mathrm{~m}$ pour les segments orientés est-ouest, avec, pour celui du sud - le seul qui ait conservé une partie de cette élévation supérieure - deux contreforts accolés à l'extérieur de 0,6 sur $0,9 \mathrm{~m}$ et distants de $2,4 \mathrm{~m}$, identiques à ceux de la curie. On a 
pu dégager ce mur sur une longueur de 2,6 m au-delà du deuxième contrefort sans en rencontrer à nouveau, ce qui laisse penser que l'espacement de $2,4 \mathrm{~m}$ ne vaut que pour l'extrémité de la façade. Les parements intérieur et extérieur de l'élévation supérieure du mur sud sont en petit appareil régulier, tandis qu'à la base, seule la face interne des murs est cn petit appareil ; de plus, le mur extérieur présente des joints en ruban analogues à ceux observés sur la curie.

L'espace intérieur est refendu par un mur nordsud de $0,9 \mathrm{~m}$ de largeur, soigneusement parementé en petit appareil sur ses deux faces, qui définit un compartiment allongé de 9,4 sur 2,4 m à l'extrémité du cryptoportique, et dans lequel est ménagé un seuil de $2,35 \mathrm{~m}$ contre la paroi nord du sous-sol. Deux trous de boulin espacés de $2,03 \mathrm{~m}$ sont encore visibles à $1,2 \mathrm{~m}$ au-dessus du sommet des fondations de ce mur; ils sont bouchés du côté ouest. L'exploration vers l'ouest n'a pas été suffisante pour permettre de localiser une éventuelle ligne de piliers axiaux. Le mur nord-sud qui ferme le cryptoportique à l'est se prolonge de $1,95 \mathrm{~m}$ au nord de l'angle de la galerie et se raccorde avec une plate-forme maçonnée qui correspond à la pile supportant la colonne de l'exlrémité occidentale du portique (cf. la restitution de la fig. 15).

\section{Nivellement et stratigraphie}

Pour l'extérieur de la galerie, les données recueillies ont déjà été exposées dans le paragraphe consacré à la description du portique (cf. supra, p. 123). La plate-forme qui marque le sommet de la partie basse des murs du cryptoportique, située entre les cotes 343,15 et 343,30 , devait être largement remblayée si l'on situe le niveau de circulation du por-

Fig. 19 - Coupes transversale A-B et longitudinale C-D-E-F de l'extrémité sud-est de la galerie à cryptoportiques (localisées sur la fig. 12).

1 : sable grossier (sol naturel); 2 : terre brune avec scories et quelques tessons gaulois (remblai lié à la construction de la galerie); 3 : calcaire jaune concassé avec épandage de mortier au sommet (sol de travail); $3^{\prime}$ : blocage (granite rose) lié par du mortier pulvérulent ; 4 et $4^{\prime}$ : sable grossier mêlé de débris de calcaire jaune (remblai); 5 : terre limoneuse grise (apport naturel par ruissellement ?); $5^{\prime}$ : terre limoneuse grise (prolongement de 5 ?); $5^{\prime \prime}$ : épandage de charbon de bois ; 6 : terre limoneuse grise (apport naturel par ruissellement); $7:$ sable fin (remblai); $8:$ sable grossier mêlé d'éclats de pierre et de mortier (remblai); $9:$ niveaux d'occupation médiévaux et moderne; 10 : terre grise gravillonneuse (niveau d'occupation gaulois); 11 : gravier grossier (comblement de la tranchée de fondation du mur périphérique de la galerie et remblai lié à la construction du portique); $12:$ calcaire jaune concassé (remblai et sol de travail liè à la construction du portique); $13:$ sol de terre battue (sol de la galerie inférieure); 14: tuiles, bois calciné et fragments d'enduit mural (niveau d'incendie); $15:$ débris de mortier et d'enduit mural (remblai de destruction); $16:$ blocs de maçonnerie effondrés (remblai de destruction). $\mathrm{A}$ : enduit égalisé $; \mathbf{A}^{\prime}$ : enduit lissé en surface.

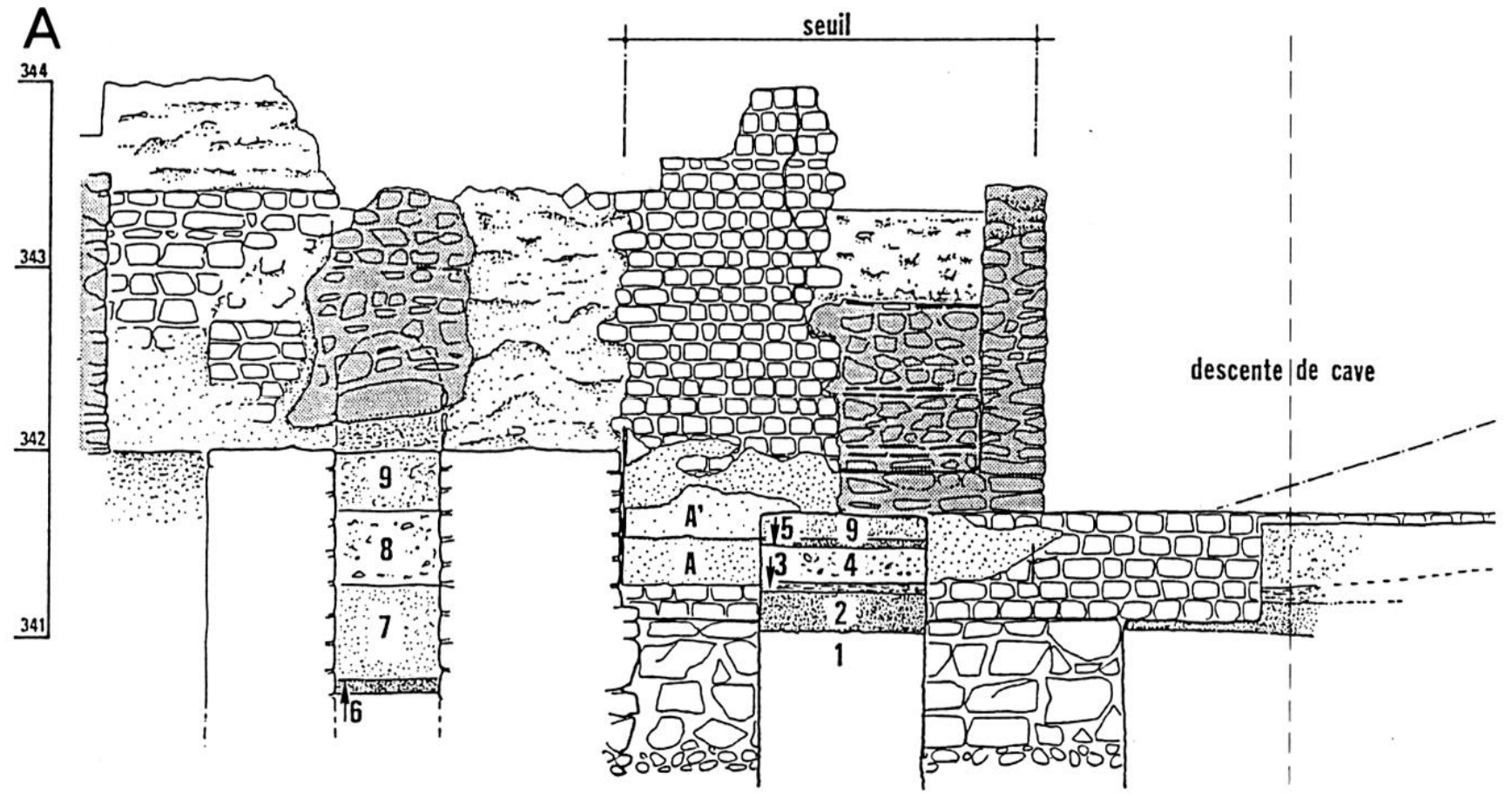


tique aux environs de la cote 344,0 . A l'appui de cette hypothèse, on notera que la surface de cette plate-forme est irrégulière sur une partie du tracé du segment nord-sud, et localement (au sud) recouverte de remblais de gravier alternant avec des épandages de mortier jusqu'à la cote 343,95 (fig. 19, couche 11). Plus au nord, elle est recouverte par un épandage de calcaire jaune concassé (fig. 19, couche 12). On remarque en particulier qu'une pellicule de ce matériau est intercalée entre la plate-forme et la première
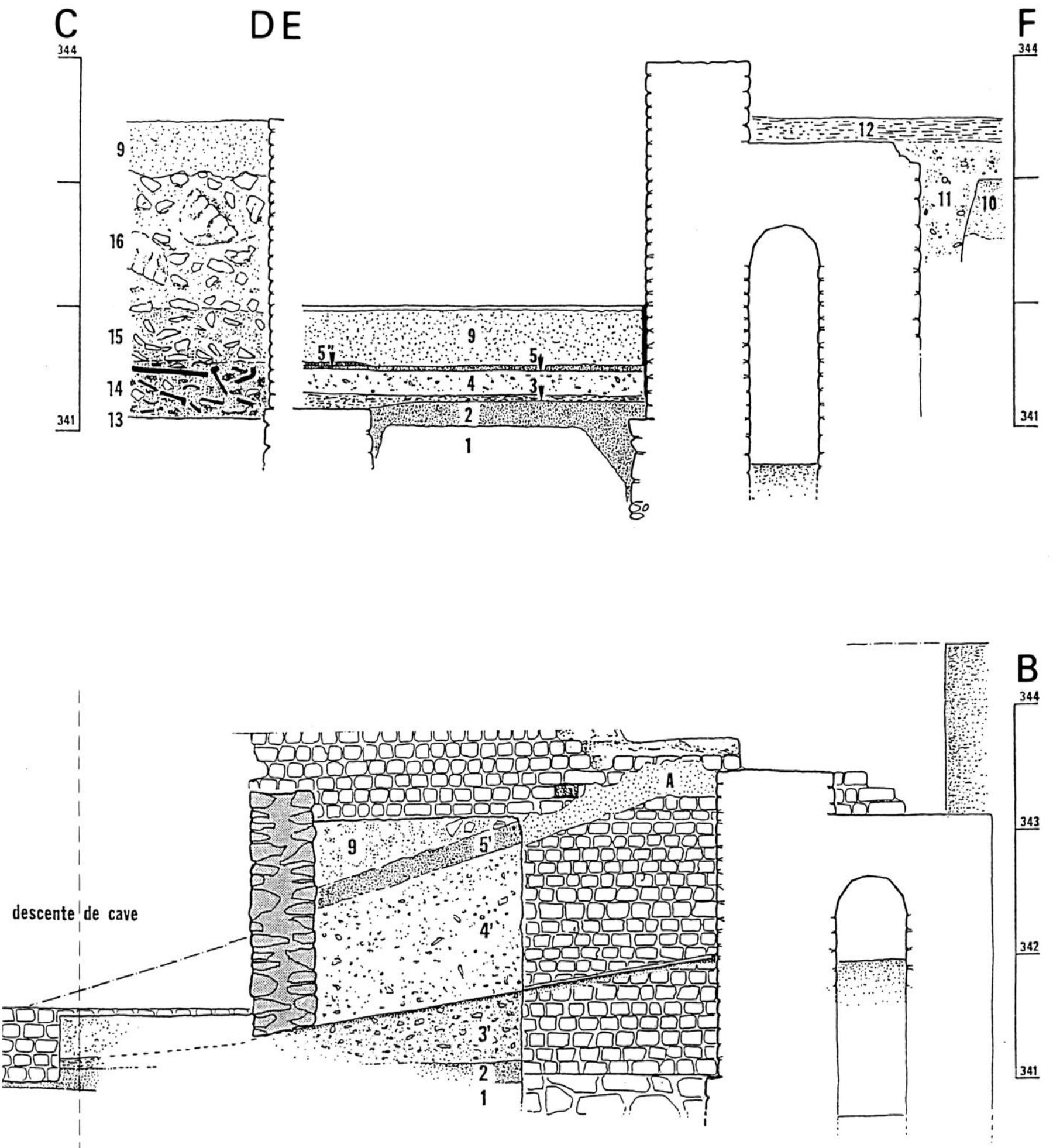
assise de l'élévation supérieure du mur nord-sud, dans sa partie septentrionale : on en conclura que cet épandage, qui recouvre l'ensemble du portique, a été mis en place avant l'achèvement du bâtiment à cryptoportique. Voilà donc qui fournit un indice supplémentaire de la simultanéité de la construction du portique et du cryptoportique, tout comme l'encastrement de la dernière pile du portique dans un massif de maçonnerie manifestement bâti en même temps que la base du cryptoportique.

Les indications relatives à la stratigraphie de l'intérieur de la galerie sont beaucoup plus nombreuses. Dans sa partie nord, le conduit est remblayé sur environ $1,5 \mathrm{~m}$. Son sol est matérialisé par un épandage de mortier. Il est recouvert par une couche de limon peu épaisse $(0,05 \mathrm{~m})$ (fig. 19, couche 6$)$, puis par une couche de sable fin qui a livré de nombreux ossements humains sans connexions anatomiques, mais aucun mobilier qui puisse assurer leur datation (couche 7). Le sommet du comblement est constitué d'un remblai de gravier et de débris de mortier qui correspond certainement à la démolition de la voûte (couche 8).

Un niveau antique assez épais était conservé sous le sol de la cave dans la partie nord de la pièce allongée limitée par le mur de refend. La base de l'élévation des murs en petit appareil est située entre les cotes 341,05 et 341,15 (soit 0,4 à $0,5 \mathrm{~m}$ en dessous du sol de la cave). Les fondations, plus larges de $0,15 \mathrm{~m}$ (sauf pour le mur de refend), sont en appareillage irrégulier de gros blocs de granite rose et reposent sur un lit de galets; elles ont une hauteur de $0,6 \mathrm{~m}$. Le seuil maçonné est situé à la cote 341,18 mais est surmonte de plusieurs horizons antiques qui laissent supposer que le niveau de circulation était situé à une cote supérieure. Il est d'abord recouvert, ainsi que la partie nord de la pièce, par un remblai de gravier mêlé de débris de calcaire jaune épais de $0,15 \mathrm{~m}$, dont la surface est localement recouverte par un épandage de mortier (fig. 19, couche 3) : il s'agit d'un niveau de travail à partir duquel a été mis en place un enduit mural sommaire (non lissé et de couleur grise) encore conservé sur une hauteur de $0,5 \mathrm{~m}$. $\mathrm{Ce}$ sol est recouvert par un nouveau remblai de gra vier et de calcaire jaune broyé, épais de $0,2 \mathrm{~m}$ (couche 4) et scellé par une mince couche limoneuse tassée (couche 5), également recouverte par endroits de traces de mortier, et dont le niveau correspond à la base d'une deuxième couche d'enduit, cette fois blanche et soigneusement lissée, retrouvée en place dans l'angle nord-est de la pièce. Aucun autre horizon antique n'a été observé au-delà, sinon peut-être une couche lenticulaire très charbonneuse au-dessus du seuil (couche $5^{\prime \prime}$ ). On considérera donc que le sommet de cette couche limoneuse, situé à la cote 341,6 , correspond au niveau de circulation antique.

Le comblement primitif de la partie sud de la même pièce, située en dehors de l'emprise de la cave, était conservé jusqu'au niveau d'arasement des murs, soit sur une hauteur de $2 \mathrm{~m}$ environ. La stratigraphie rencontrée est très différente de celle de sa partie nord, puisque toutes les couches de terrain rencontrées présentent un pendage très prononcé vers le nord. Une première couche, dont la base est au niveau du sommet des fondations, est constituée d'un blocage de fragments de granite rose de petit module (dont un amas de moellons d'appareil) noyé dans un mortier blanc sans cohésion (couche $3^{\prime}$ ). Le sommet de cette couche, matérialisé par une surface de mortier régulière dont le contour, le long des murs, est souligné par un joint carré de 2 à $3 \mathrm{~cm}$, est plan mais nettement incliné vers le nord. Elle est surmontée par un épais remblai de gravier grossier dont le niveau supérieur, également plan, présente un pendage encore plus prononcé (couche $4^{\prime}$ ). Le sommet de ce remblai correspond à la base d'un enduit mural peu soigné (le prolongement de celui qui vient d'être décrit) observé sur les deux murs latéraux de la pièce et bien conservé sur une hauteur de $0,2 \mathrm{~m}$ (fig. 20), qui correspond à l'emprise d'un nouveau remblai de terre grise (couche $5^{\prime}$ ). Le comblement supérieur, hétérogène (couche 9), ne parait pas antique.

Il convient de proposer tout de suite une interprètation aux observations qui précèdent. La présence de ces couches en plan incliné laisse évidemment penser à une descente permettant l'accès à la galerie inférieure. Les accès aux cryptoportiques de forum de plan en $U$ sont en effet presque toujours situés à l'extrémité des galeries latérales (ainsi à Conimbriga, Bavay, Reims, Trèves...). Les dimensions mème de la pièce correspondent également aux normes habituelles des cages d'escalier au Haut Empire (2,4 sur 6,4 m dans le premier état de Bavay, 2,5 sur $8,0 \mathrm{~m}$ à Trèves). Si la présence d'un enduit mural dont la base inclinée se retrouve sur les deux parois latérales laisse penser que l'escalier d'accès n'avait qu'une volée de marches de 2,4 m de largeur (qui correspond d'ailleurs à la largeur du seuil), il est peu plausible que la pente des remblais et de cette limite d'enduit corresponde à la pente d'origine de l'escalier, puisque la dénivellation est de $2 \mathrm{~m}$, valeur beaucoup trop faible par rapport à sa longueur de $7 \mathrm{~m}$. Il est plus logique de penser que l'accès à l'escalier se faisait depuis le portique par une porte percée dans le mur de façade est du cryptoportique. En res- 


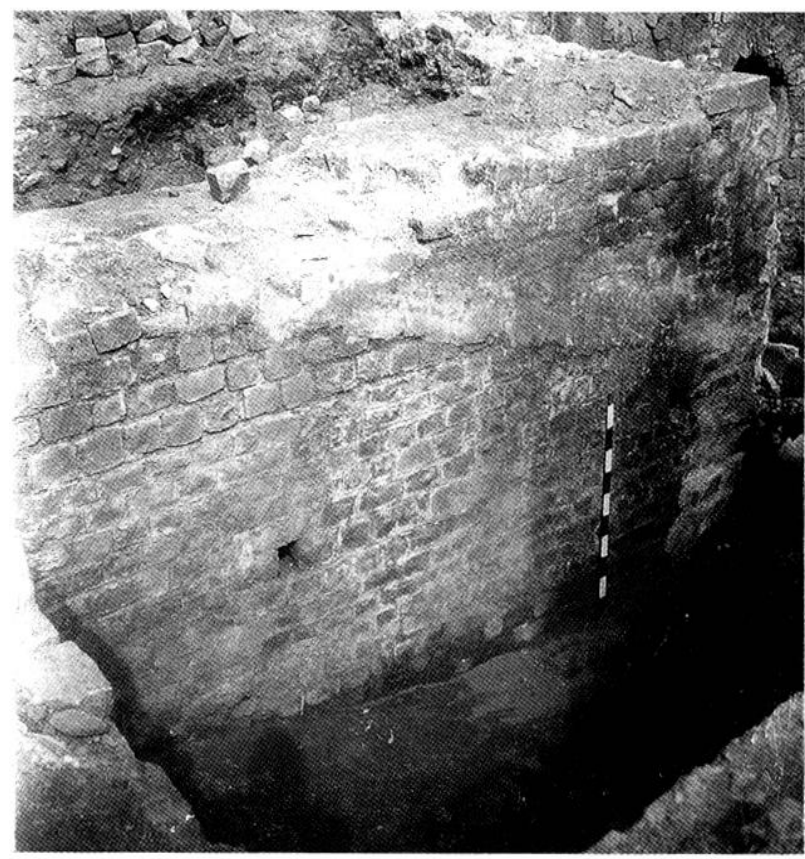

Fig. 20 - Parement est du mur de refend de l'aile sud du cryptoportique (1978). Le mur est recouvert d'un enduit dont la base a un pendage prononcé vers le nord, dénotant l'existence d'un ancien accès en plan incliné vers la galerie inférieure du cryptoportique.

tituant à cette porte une largeur identique à celle du seuil inférieur $(2,4 \mathrm{~m})$, on obtient une longueur effective d'escalier de $4,6 \mathrm{~m}$, pour une dénivelée de $2,5 \mathrm{~m}$ environ (différence de niveau entre le portique et la galerie inférieure), soit une pente beaucoup plus normale (divisible par exemple en douze marches de $21 \mathrm{~cm}$ de volée et $38 \mathrm{~cm}$ de giron). L'accès à la galerie supérieure pouvait alors s'effectuer par un escalier parallèle à celui-là, et auquel on accédait par une deuxième porte percée plus au nord dans le mur est du cryptoportique, dans le prolongement du portique (cf. restitution fig. 15).

On n'a pu pratiquer qu'une tranchée de sondage étroite dans le comblement de la galerie inférieure du cryptoportique épargné par la cave à l'ouest du mur de refend nord-sud. Néanmoins, l'observation de couches de destruction non perturbécs apporte des indications très précieuses sur la nature de l'élévation de la galerie. Le sol d'origine, en terre battue et situé à un niveau inférieur à celui du seuil (cote 341,1 , est en effet directement recouvert par les débris de la toiture incendiée (fig. 19, couche 14): tuiles mêlées de fragments de poutres calcinées, ce qui montre indubitablement que la galerie inférieure n'était pas voûtée. Ce niveau d'incendie était surmonté par une épaisse couche de gravats (couches 15 et 16), dont la base contenait un grand nombre de fragments d'enduits et le sommet des blocs de maçonnerie effondrés, parfois très volumineux. Deux types d'enduits peuvent être distingués. Le premier n'est constitué que d'une couche homogène de mortier assez gris à inclusion de sable grossier, épais de $3 \mathrm{~cm}$ en moyenne et soigneusement lissé, qui correspond au revêtement de la galerie inférieure, puisque d'importants fragments ont été retrouvés en place sur les parois; le second est constitué de deux couches de mortier, la plus profonde identique à celle qui vient d'être décrite, et la couche superficielle, plus mince $(0,5 \mathrm{~cm})$, constituée de mortier blanc à nombreuses inclusions de feldspath. Sa surface est parfaitement lissée et présente des restes de décoration peinte, qu'il est toutefois impossible de caractériser précisément compte tenu de l'exiguité de la surface fouillée (Allag, 1985) : elle devait du moins comporter d'importants panneaux rouge sur fond blanc cernés par une bordure blanche rehaussée de trois traits surpeints (deux violets encadrant un vert), dont quelques fragments présentent également des surpeints verts, mais aussi des panneaux bleus dont de nombreux fragments ont èté retrouvés, présentant parfois aussi des traces de décor surpeint. Une dernière particularité à mentionner est la fréquence des graffiti sur les fragments du second type.

\section{Éléments de datation}

Le mobilier retrouvé en position stratigraphique est très peu abondant. La base de la couche d'incendie de la galerie, explorée sur seulement $2 \mathrm{~m}^{2}$, a livré quelques ossements animaux et un petit lot de tessons de céramique (environ 90), dont les éléments les plus caractéristiques sont les suivants :

- céramique sigillée: un fond de vase haut, (fig. 21, no 1), un petit fragment de paroi de bol Drag. 37 à décor moulé fruste, un fragment de paroi de forme basse lisse, le tout de pâte et vernis rouge soutenu typiques des productions du Centre-Gaule;

- céramique à couverte micacée : trois fragments de paroi d'une coupe évasée (fig. 21, no 2), trois fragments de paroi d'une cruche à col tronconique large, un fragment de jatte profonde à lèvre en bandeau ( $\left.n^{\circ} 3\right)$;

- céramique à cuisson réductrice-oxydante : un fragment de cruche à pied cintré (fig. 21, no 4), un fragment de cruche à col tronconique large, un rebord de vase ovoïde ( $\left.n^{0} 5\right)$, des fragments de deux écuelles tripodes basses, deux fragments d'une écuelle à lèvre droite en bandeau ( $\left.n^{\circ} 6\right)$, une lampe à suif $\left(n^{\circ} 7\right)$;

- céramique à cuisson réductrice : deux fragments de paroi de vase haut, décorés à la molette (fig. $21, \mathrm{n}^{\mathrm{os}} 8$ et 9 ). 

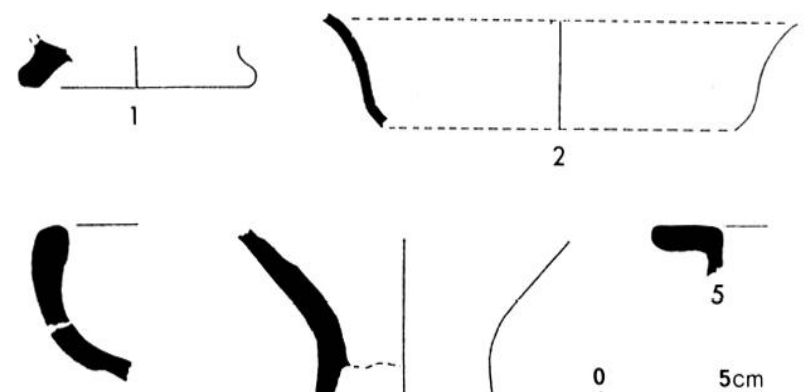

3
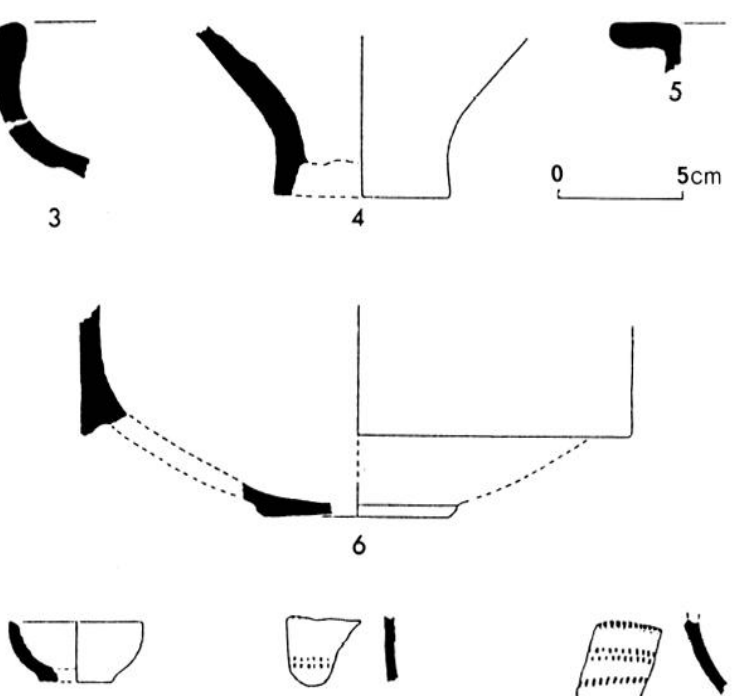

7

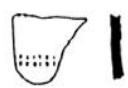

8

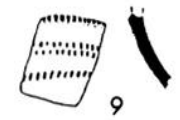

Fig. 21 - Mobilier provenant du remplissage de la galerie, dans les niveaux d'incendie (fig. 19 , couches 14 et 15 ).

Certains de ces objets ne peuvent pas être datés avant la fin du $I^{\mathrm{e}} \mathrm{s}$. (en particulier la jatte $\mathrm{n}^{\circ} 6$, dont la forme imite celle des mortiers Drag. 45, et les formes micacées).

Par ailleurs, une couche inférieure de la descente (couche 3 '), contemporaine de la construction (ou d'une réfection) de l'escalier, a livré, outre des fragments d'un pichet à engobe rouge, typique des productions de Lezoux au I $^{\text {er }}$ s., un as de Domitien (Cohen 583). I a présence de moellons de petit appareil dans la même couche laisse possible l'hypothèse d'un remblai à mettre en rapport avec un remaniement de l'édifice primitif.

\section{Autres observations}

Environ les deux-tiers des caves de l'îlot qui recouvre actuellement la galerie à cryptoportiques ont pu être visités (cf, infra, p. 161). Des vestiges de constructions antiques ont pu être identifiès dans cinq d'entre elles.

Ces observations ont pu être complétées par une petite fouille de sauvetage dans l'angle sud-ouest interne de la galerie, en 1980 , et par le suivi de travaux de voirie en 1991 (fig. 22).

Nous nous bornerons ici à décrire les vestiges qui ont été observés. Les problèmes posés par leur raccordement et la restitution du plan d'ensemble seront développés ultérieurement.

\section{Mur de façade intérieur}

L'aile sud est la mieux connue à cause de la fouille de son extrémité est. On sait de plus que le mur de façade nord de cette aile a été dégagé sur $17 \mathrm{~m}$ et fortement arasé en 1967, lors de la reconstruction d'un immeuble (fig. 2 , point 4). Le même mur peut encore être observé dans une cave située immédiatement plus à l'ouest (fig. 2, point 5 et fig. 22) : la base élargie du mur antique, réutilisée en fondation, émerge de $30 \mathrm{~cm}$ au-dessus du sol de cette cave, à la cote 342,7 .

L'angle intérieur sud-ouest de la galerie a pu être dégagé en 1980 , lors de la reconstruction d'un immeuble (fig. 2, point 6); les vestiges antiques ont été cette fois épargnés. Le bâtiment existant s'appuyait sur les deux murs antiques et sa cave était exactement insérée dans leur angle. De l'élévation des murs ne restait conservée que la partie basse élargie. La façade sud de l'aire circonscrite par la galerie a été dégagée sur $9 \mathrm{~m}$ à partir de l'angle, la façade ouest sur $8 \mathrm{~m}$. Le sommet des maçonneries était à la cote 343,25 , qui correspond assez précisément à celle relevée dans l'angle nord-est de l'aile sud $(343,30)$. Des sondages dans l'aire interne ont permis de retrouver une stratigraphie tout à fait comparable à celle observée à l'emplacement du portique :

- le sol primitif est à la cote 341,8 , soit une dénivelée de $1,2 \mathrm{~m}$ depuis l'extrémité ouest du portique. Ce sol scellait également un niveau d'occupation des III $\mathrm{e}_{-\mathrm{I}^{\mathrm{er}}}$ s. avant J.-C. Comme pour la fouille de 1978, on a pu constater que l'occupation est très diffuse après le milieu du $\mathrm{I}^{\mathrm{er}} \mathrm{s}$. avant J.-C. (l'élément datable le plus récent de ces niveaux est une lèvre d'amphore Dr. 7/11; cf. Vaginay, Guichard, 1988, fig. $\left.11 \mathrm{n}^{\circ} 32\right)$;

- ce sol est recouvert par un épais remblai de gravier qui n'a livré d'autre mobilier que quelques tessons gaulois déplacés, surmonté par un mince épandage de calcaire jaune pilé et tassé, dont le sommet est à la cote 343,3 .

Ce remblai s'appuie contre les murs antiques, tandis que l'aménagement de sol à son sommet est situé sensiblement au même niveau que l'extrados du conduit d'assainissement et à $0,2 \mathrm{~m}$ en dessous de la cote minimale restituable pour la place centrale en avant des portiques. Aucun autre horizon antique n'a pu être repéré entre ce sol et le sol actuel (à la cote 344,8$)$.

Le mur de façade interne de la galerie a encore pu être repéré à partir d'une cave située rue Gambetta (fig. '2, point 7 et fig. 22). I a limite sud de cette 


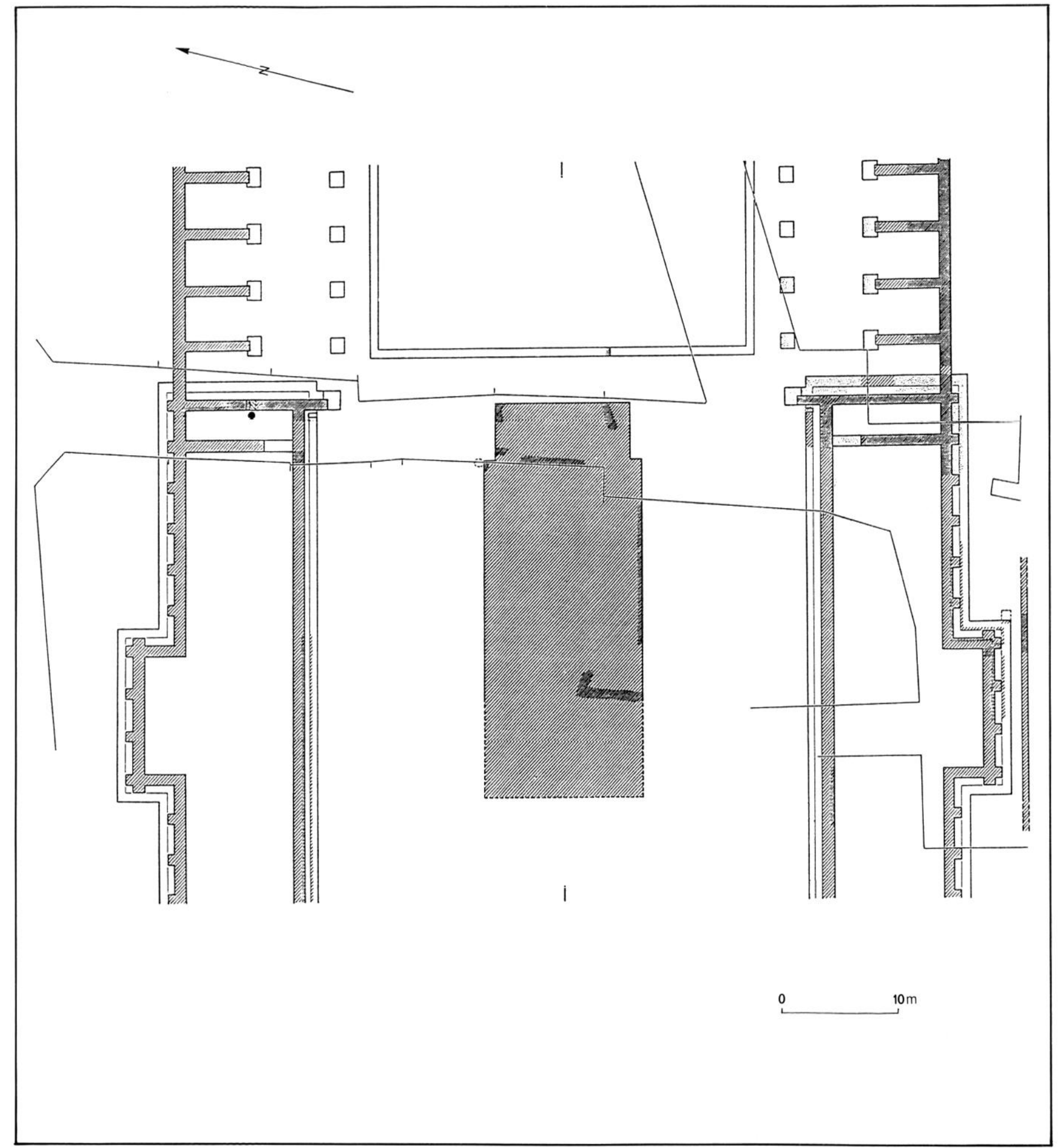

Fig. 22 - Synthèse des observations sur la partie orientale de l'aire sacrée; la pastille noire indique l'emplacement d'un bloc de grand appareil ; en noir, les maçonneries antiques observées. 
parcelle suit le tracé du rempart médiéval (vraisemblablement encore partiellement conservé en élévation) qui lui-même s'appuie sur le mur antique, ainsi que le remarquait déjà Roux (1851, p. 47). L'angle intérieur nord-ouest de la galerie est nettement visible : la voûte du conduit d'assainissement a été arrachée sur une longueur de $9 \mathrm{~m}$ à partir de l'angle, le long du mur nord. Trois arcs de décharge ont ensuite été construits dans l'encoche ainsi ménagée. Leur facture n'est certainement pas antique; ils paraissent plutôt faire partie de l'élévation du rempart. Ces arcs permettent du moins d'accéder facilement au conduit, qui n'est pas totalement comblé. On a ainsi pu reconnaître, en y cheminant, toute la longueur de la façade ouest de l'aire interne (le conduit est obturé par de la terre quelques mètres avant l'angle sud-ouest), ainsi que sa façade nord sur $22 \mathrm{~m}$ (le conduit est également comblè au-delà). La facture des maçonneries est parfaitement homogène et identique à celle observée dans l'extrémité de l'aile sud en 1978.

Enfin, la réfection de la rue du Palais a permis de dégager superficiellement l'extrémité nord-est du cryptoportique et de vérifier sa symétrie parfaite avec l'extrémité sud-est (fig. 22). Ces travaux ont permis d'observer la pile extrême du portique de la place centrale (cf. supra, p. 127) ainsi qu'un bloc de grand appareil en calcaire jaune encastré dans la maçonnerie du mur pignon de la galerie. Ce bloc pourrait correspondre à un jambage de l'ouverture supposée entre le portique et la galerie haute du cryptoportique (fig. 15).

\section{Mur de façade extérieur}

Roux situait le conduit extérieur à $16 \mathrm{~m} \mathrm{du}$ conduit intérieur, à l'exception des extrémités des ailes latérales où il indique que cette largeur est réduite à $12 \mathrm{~m}$ (ainsi que l'ont confirmé nos travaux). En dehors de la fouille de 1978, le conduit du mur de façade extérieur a été observé en quatre points, trois dans l'aile sud (fig. 2 , points 8,9 et 10) et un dans l'aile nord (fig. 2, point 13). Au point 8, le conduit d'assainissement a pu être repéré précisément dans la cave d'un immeuble du $x^{*}$ s., où il est apparent sur une longueur de $5 \mathrm{~m}$. Il est exactement dans l'alignement du tronçon dégagé plus à l'est lors de la fouille de 1978, en contradiction avec la description de Roux. Dans la cave mitoyenne (point 9), aménagée au $\mathrm{XIX}^{\mathrm{e}} \mathrm{s}$, une banquette maçonnée et recouverte d'enduit semble correspondre à son prolongement. Sur la place du Puits de l'Aune (point 10), la réfection du réseau de distribution d'eau, en avril 1991, a mis au jour un court segment du mur de façade. L'exploration du conduit d'assainissement à partir de ce point a montré qu'il s'agissait de l'angle sud-est d'une exèdre profonde de $3,60 \mathrm{~m}$, valeur proche de l'élargissement de $4 \mathrm{~m}$ décrit par Roux, qui explique l'erreur qu'il a commise en restituant le plan d'ensemble du cryptoportique. La longueur explorėe du conduit montre aussi que l'exèdre est large d'au moins $7,5 \mathrm{~m}$. Trois regards annénagés à la base de la voûte, identiques à celui observé dans l'angle nord-est de la branche sud du cryptoportique en 1978, s'ouvraient aux angles de l'exèdre, l'un vers l'extérieur, les deux autres vers l'intérieur. Un contrefort d'angle en petit appareil, large de $0,90 \mathrm{~m}$ et saillant de $0,60 \mathrm{~m}$, a également été dégagé par ces travaux. L'examen du parement d'un puits médiéval plaqué contre le mur antique (celui qui a donné son nom à la place) a permis de constater que les fondations s'enfonçaient jusqu'à la cote 341,0 . On a aussi observé un mur antique parallèle au grand axe du forum et distant de $2,2 \mathrm{~m}$ dı mur de l'exèdre. Large de $0,60 \mathrm{~m}$, il repose sur une fondation située au même niveau que la plate-forme qui surmonte le conduit d'assainissement. Il est lié avec un mortier semblable à celui du cryptoportique mais est constitué de petits moellons beaucoup plus irréguliers, dont quatre assises étaient conservées. Entre les deux murs était encore en place une épaisse couche de démolition antique $(0,4 \mathrm{~m})$ qui reposait directement sur la plate-forme et qui a livré, outre de nombreux fragments de legulae et des moellons de petit appareil, un tesson de sigillée de Gaule du Centre à décor excisé datable du ${ }_{11}{ }^{\mathrm{e}} \mathrm{s}$.

Nous avons peu de renseignements sur le mur extérieur des autres façades : sur le côté nord il serait situé exactement à l'emplacement du fossé de l'enceinte médiévale et a donc dû être détruit depuis longtemps, à l'exception de son extrémité est qui sert de fondation au rempart médiéval, encore en partie en élévation, et où Roux localisait une porte d'accès au conduit. Sur le côté ouest, toutes les constructions datent du siècle dernier et ont dû également en faire disparaître les vestiges.

Il est finalement aisé de restituer la disposition de ce mur extérieur, en corrigeant les erreurs de Roux. Le cryptoportique a une largeur dans œuvre de 9,4 m et est pourvu d'exèdres saillantes de 3,6 m. La restitution de deux exèdres latérales symétriques, distantes de 19,8 m de l'extrémité orientale du cryptoportique, et d'une exèdre axiale, selon un modèle bien attesté en Gaule (à Bavay, à Nyon et à Trèves), avec diverses variantes, paraît très vraisemblable.

\section{Temple}

On n'en connaît que la platée de fondation, dont le parement sud a pu être relevé dans une cave 
(fig. 2, point 12), où il est visible, mais malheureusement enduit, sur une longueur de $10 \mathrm{~m}$ et une hauteur de $1,5 \mathrm{~m}$ : il s'agit d'un immeuble moderne dont la cave projetée a dû être réduite à cause du massif de maçonnerie antique qui s'était révèlé indestructible au marteau-piqueur.

Il a depuis (en 1984) élé possible d'étudier la facture de ce massif, à l'occasion du creusement de tranchées de fondation dans la parcelle située immédiatement plus à l'ouest (fig. 2, point 11 et fig. 22).

C'est une maçonnerie pleine, actuellement arasée à la cote 344,73 au point le plus haut, que les tranchées ont permis de reconnaitre sur une largeur de $7 \mathrm{~m}$ à partir du parement sud. Le parement n'a pu être observé qu'en deçà du niveau du sol de calcaire jaune qui recouvre l'aire circonscrite par la galerie (le sommet du parement conservé est à la cote $343,46)$. Il est constitué de petit appareil irrégulier identique à celui des autres parements des bâtiments du forum destinés à être remblayés.

L'arasement irrégulier du massif montre clairement que la construction s'est effectuée en plusieurs étapes, ainsi qu'on a pu l'observer pour d'autres éléments du forum : il a d'abord été appareillé jusqu'à la cote 343,54 , à laquelle a ensuite été remblayée l'aire périphérique, avec aménagement d'un sol (provisoire?) de calcaire jaune pilé dont on retrouve des traces à l'interstice entre la base du massif et l'élévation ultérieure, situé à la même cote que lui, ce qui témoigne de l'existence d'un délai suffisant entre les deux phases de construction pour que ces salissures aient eu le temps de se déposer sur la maçonnerie.

La réfection de la rue du Palais a enfin permis de repérer l'extrémité est de la platée (fig. 22) et de préciser son mode de construction : ses fondations, dégagées par un puits moderne, s'enfoncent jusqu'à la cote 341,0 . Les mêmes travaux ont montré qu'elle était prolongée du côté est par un massif de facture semblable mais plus étroit $(12,1 \mathrm{~m}$ contre $14,1 \mathrm{~m}$ pour la platée) et long de $4,6 \mathrm{~m}$, qui correspond certainement à l'emprise de l'emmarchement du temple. Un tronçon nord-sud de l'emmarchement qui délimitait la place centrale a probablement été repéré dans une cave mitoyenne de la rue du Palais, à $3,8 \mathrm{~m}$ en avant de celui du temple, sous la forme d'un bloc de calcaire jaune de 0,75 sur $0,45 \mathrm{~m}$ de section reposant sur une fondation massive, engagé dans la paroi de la cave.

\section{L'ÉGOUT DU FORUM}

Déjà reconnu par Roux (1851, p. 46), deux sections en ont été dégagées en bordure de la rue de Roanne lors de fouilles de sauvetage précédant la reconstruction d'un îlot d'habitations (Maison de la Commune; fig. 23, point 1), et celle de l'aile sud de l'hôpital, en 1981 (fig. 23, point 2).

\section{Description}

L'égout mis au jour est un massif de maçonnerie de section rectangulaire, haut de $1,85 \mathrm{~m}$ (fondations exclues) et large de $1, \overline{0} 0 \mathrm{~m}$ (fig. 24). Il renferme un couloir voûté haut de $1,40 \mathrm{~m}$ et large de $0,60 \mathrm{~m}$. Sa facture est rigoureusement identique à celle du conduit d'assainissement de la galerie à cryptoportiques : la voûte, épaisse de $0,5 \mathrm{~m}$, a été construite sur un coffrage de six planches de $17-18 \mathrm{~cm}$ de largeur, les parois internes sont constituées de 17 à 18 assises de moellons de petit appareil assez irréguliers; la base du conduit est matérialisée par un mince épandage de mortier mis en place au moment de la construction. Le remplissage interne, épais de $0,8 \mathrm{~m}$ dans le tronçon méridional, est moderne jusqu'à la base, tandis que le conduit est fortement arasé dans la plus grande partie du tronçon septentrional.
Fig. 23 - Plan de situation des vestiges de l'égout d'assainissement du cryptoportique (1,2: tronçons fouillés en $1981 ; 3$ : tronçon de conduit maçonné dégagé en 1887 et localisé d'après Poncins 1887).

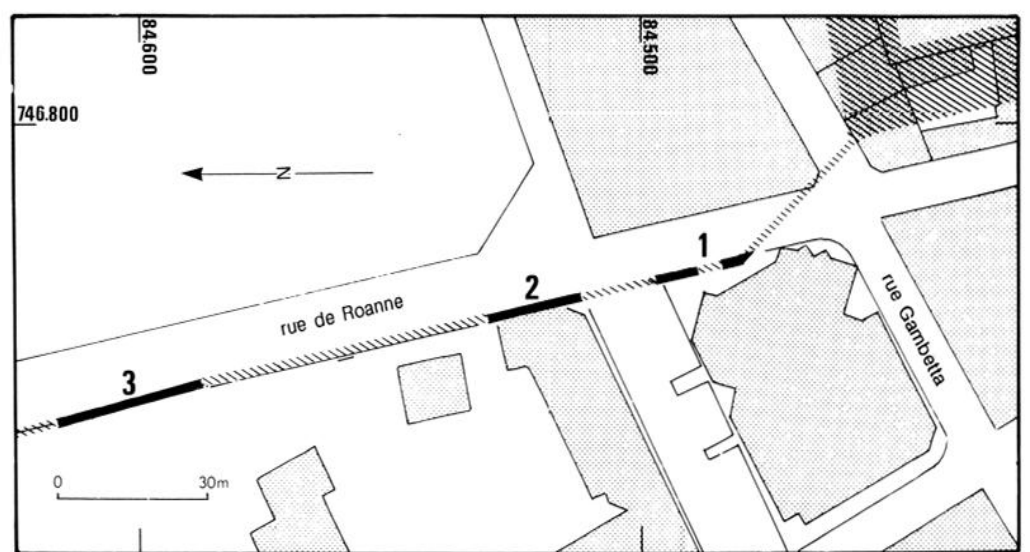


$E$

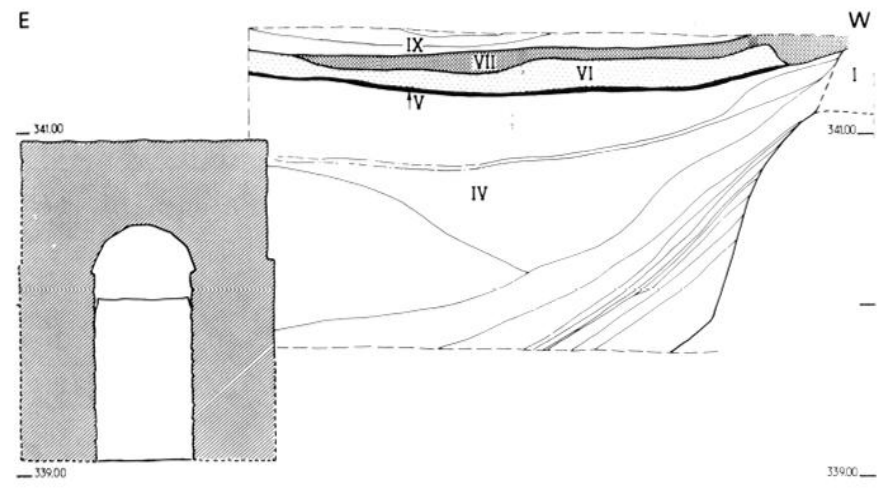

Fig. 24 - Coupe transversale de l'égout et des couches archéologiques qui lui sont liées (fouille de 1981 ; désignation des couches selon : Vaginay, Valette, 1982, fig. 14; I : niveau d'occupation du Irr s. avant J.-C.; IV : comblement de la tranchée de fondation de l'égout; $\mathrm{V}$ à $\mathrm{IX}$ : couches indurées correspondant aux niveaux de circulation successifs de la rue).

Le parement externe est appareillé sur toute sa hauteur en petits moellons irréguliers de granite rose jointoyés au mortier. Des trous de boulin sont disposés à espacements réguliers de $1,90 \mathrm{~m}$ et à $1,25 \mathrm{~m}$ de la base du parement.

Les deux tronçons repérés permettent de restituer le tracé rectiligne du conduit, quasiment parallèle à la rue de Roanne, sur $50 \mathrm{~m}$. A l'extrémité sud, la fouille a également dégagé un coude qui lui permettait de rejoindre, en biais, l'angle extérieur nordouest du forum. Ce coude est cohérent avec le plan de Roux, ce qui n'est pas le cas de la partie rectiligne de $50 \mathrm{~m}$ (Roux trace un second coude vers l'ouest à environ $16 \mathrm{~m}$ du premier - cf. fig. 17).

\section{Nivellement}

Le niveau supérieur du massif maçonné aux points extrèmes de la partie dégagée ne présente qu'une faible pente vers le nord (cote 341,08 au sud, 340,88 à $35 \mathrm{~m}$ plus au nord). La façon dont l'égout se raccorde avec le conduit d'assainissement du forum n'est pas connue. Roux affirme avoir parcouru ce "dégorgeoir" sur une "assez grande longueur", mais qu'il l'a trouvé muré "en approchant du point d'intersection des angles" (angle nord-ouest du forum), ce que l'on a effectivement pu vérifier. Néanmoins la similitude des modes de construction et l'inflexion vers l'est de son axe laissent peu de doutes quant à sa fonction. Les données de nivellement imposent dailleurs certaines restrictions sur la nature de ce raccordement. Le fond du conduit d'assainissement dans l'angle sud-est de la galerie à cryptoportiques est en effet à la cote 340,7 , qui est quasiment l'altitude du sommet de la voùte de l'égout
$(340,6)$. Même si l'on doit supposer que le sol du conduit d'assainissement était en légère pente en direction de l'exutoire, il demeure une forte dénivelée de près de $1,5 \mathrm{~m}$ entre l'égout et le conduit. L'origine de cette dénivelée s'explique facilement: l'égout étant situé dans l'axe d'une rue antique qui longeait le forum (cf. infra, p. 148) et dévalait vers le nord en direction de la vallée de la Loise, son sommet devait être situé en dessous du niveau de circulation, situé aux alentours de la cote 341,5 au moment de sa construction. Le raccordement devait se faire au moven d'un infléchissement du niveau du sol du conduit d'assainissement dans son angle nordouest, par un puisard ou un plan incliné.

L'égout drainait certainement l'eau jusqu'à la Loise. Une section supplémentaire en a vraisemblablement été dégagée en 1887 le long de la même rue, à $65 \mathrm{~m}$ au nord de celle repérée en 1981 (Poncins, 1888; fig. 23, point 3). Ce tronçon, long de $30 \mathrm{~m}$, qui avait alors été considéré comme un aqueduc, est en effet sensiblement dans l'axe de l'égout si l'on se réfère aux relevés de l'époque, et "ses deux murs latéraux, épais de $0,45 \mathrm{~m}$, étaient arasés au même niveau, sans vestiges d'arrachement pouvant faire supposer l'existence d'une voûte. La profondeur du canal était de $0,70 \mathrm{~m}$, sa largeur de $0,55 \mathrm{~m}$. Le radier était formé de tuiles à rebords de très grande dimension, 0,53 sur $0,34 \mathrm{~m}$, placées en travers et juxtaposées, les rebords en dessous. Ces tuiles reposaient. sur un lit de béton hydraulique de $0,08 \mathrm{~m}$, établi luimème sur une couche de $0,10 \mathrm{~m}$ de pierres cassées". Le module du conduit (largeur du canal et des parois latérales) correspond à celui de l'égout. Le sol maçonné peut s'expliquer par la plus grande pente du terrain à cet emplacement, qui nécessitait de pallier l'érosion due à la circulation de l'eau.

\section{Données stratigraphiques}

La fouille de niveaux d'occupation liés à la construction de l'égout en 1981 a livré un mobilier datable avec précision qui apporte les éléments les plus tangibles pour la datation de la mise en chantier du forum. Ces données stratigraphiques, déjà décrites en détail (Vaginay, Valette, 1982), seront seulement résumées ici.

On a en effet pu observer que l'egout avait été installé dans une large tranchée dont le comblement était scellé par des couches successives de terrain induré correspondant à des niveaux de circulation intense (fig. 24). Bien que leur sommet soit fortement arasé, il est très probable qu'il faille y voir, à l'image d'autres structures de voirie mieux conservées dégagées depuis lors à Feurs, la base des niveaux de cir- 
culation successifs d'une rue située dans le mème axe que l'égout sous-jacent et bordée, à l'est par le forum, à l'ouest par un quartier d'habitat qui a pu ètre partiellement dégagé. Si cette rue n'a pu ètre fouillée que sur une longueur réduite, les indications de datation sont très précises parce que certains de ses niveaux ont livré un mobilier abondant. La plus ancienne couche postérieure à la construction de l'égout précisément datable par son mobilier (fig. 24, couche VII) comportait 54 vases identifiables, dont une part appréciable (36 vases) de céramiques importées et un as de Tibère frappé en 10-11 (RIC 368). La datation qui en est proposée, autour de 20, est confortée par l'analyse des couches immédiatement sur-jacente (couche VIII, établie au cours de la trojsième décennie de notre ère) et sous-jacente (couche IIIc, vraisemblablement contemporaine de la construction de l'égout, établie pendant la première décennie de notre ère). Comme la couche VII est précédée de deux autres niveaux de sol bien constitués également postérieurs à l'égout, la construction de ce dernier doit ètre située vers 15 après J.-C. au plus tard.

\section{RESTITUTION DU PLAN D'ENSEMBLE}

\section{Raccordement des relevés; plan régulateur}

Les données rassemblées sont suffisantes pour permettre la restitution du plan au sol du monument dans sa quasi-totalité (fig. 25). Compte tenu de la façon dont onl été répérées ses différentes parties: relevés partiels, ètalés sur plus de 30 ans, de vestiges souvent détruits ou remblayés par la suite, le plan que nous proposons ne peut cependant prétendre à la mème précision que si le monument avait été dégagé en une seule fois dans son ensemble. On privilégiera les données vérifiées récemment (1988) sur les parties du. monument encore accessibles : curie, mur périphérique de la galerie à cryptoportiques, en ajustant au mieux les relevés plus anciens pour les parties qui ne sont plus visibles. Les dernières observations sur le terrain (1991) s'ajustent avec précision à la restitution effectuée antérieurement : la marge d'incertitude n'a jamais été transgressée pour les nouvelles parties mises au jour. Elles permettent en particulier de déterminer exactement la largeur du forum au niveau du cryptoportique.

L.es relevés récents ont été effectués au télémètre (positionnement des élévations actuelles sur le plan $\grave{a} \pm 0,2 \mathrm{~m}$ ) et complétés par triangulation (notamment pour les relevés de caves). Toutes les mesures ont été reportées sur un plan au 1/200".

Le positionnement précis des deux branches nord et sud du conduit d'assainissement de la galerie à cryptoportiques permet de tracer l'axe longitudinal est-ouest du monument jusqu'à la curie (avec une précision de $\pm 0,2 \mathrm{gr}$ ). Cet axe est exactement superposable à celui de la curie et quasiment parallèle au mur d'appui des cellules du portique latéral dégagé en 1978 (la déviation de $0,4 \mathrm{~m}$ pour la longucur déga gée de ce mur de $44 \mathrm{~m}$ est imputable à une erreur de relevé lors de la fouille, puisque cet écart se retrouve dans le positionnement du parcellaire moderne).

Le relevé de la partie nord de la basilique (1968), repris à partir des cotes qui y sont indiquées, montre également un léger décalage de la travée nord de la colonnade vers l'ouest $(0,4 \mathrm{~m})$, et un désaxement du mur pignon nord (de $0,3 \mathrm{~m}$ pour une longueur de $20 \mathrm{~m}$ ) par rapport à sa situation attendue, valeurs qui restent dans la marge d'erreur de ces relevés. On en conclura qu’à la précision des relevés, les diffèrentes parties de l'ensemble monumental du forum sont rigoureusement alignées.

La détermination de l'axe longitudinal permet ensuite de restituer l'ensemble du monument par symétrie.

L'incertitude avec laquelle sont connues ses dimensions et la nature des vestiges architecturaux observés (la plupart appartiennent à des fondations à l'origine enterrées) s'opposent à la recherche de la valeur exacte du (des) module(s) de base utilisé(s) pour sa construction. On peut cependant rechercher si certaines dimensions exprimées en pieds et les proportions principales du monument correspondent à des valeurs simples et donc intentionnelles, afin de comprendre le mode d'élaboration de son plan directeur ${ }^{17}$.

\section{Temple et aire sacrée}

La largeur hors tout de la galerie à cryptoportiques $(14,20 \pm 0,05 \mathrm{~m})$ correspond clairement à 48 pieds $(14,19 \mathrm{~m})$ et sa largeur dans œuvre $(9,40 \mathrm{~m})$ à 32 pieds. Le seuil observé dans la branche sud, large de $2,35 \mathrm{~m}$, correspond au quart de cette valeur (8 pieds).

La largeur de la platée de fondation du temple est de 14,1 m, dimension qui correspond à la largeur hors tout de la galerie à cryptoportiques et à l'espacement entre la galerie et le temple $(14,6 \mathrm{~m})$. Il n'est donc pas douteux que la partition de la largeur du forum en cinq modules égaux, qui correspondent à 48 pieds chacun, soit à considérer comme un élément du plan directeur.

17 La valeur de référence retenue pour la mesure du pied est de $0.296 \mathrm{~m}$. 


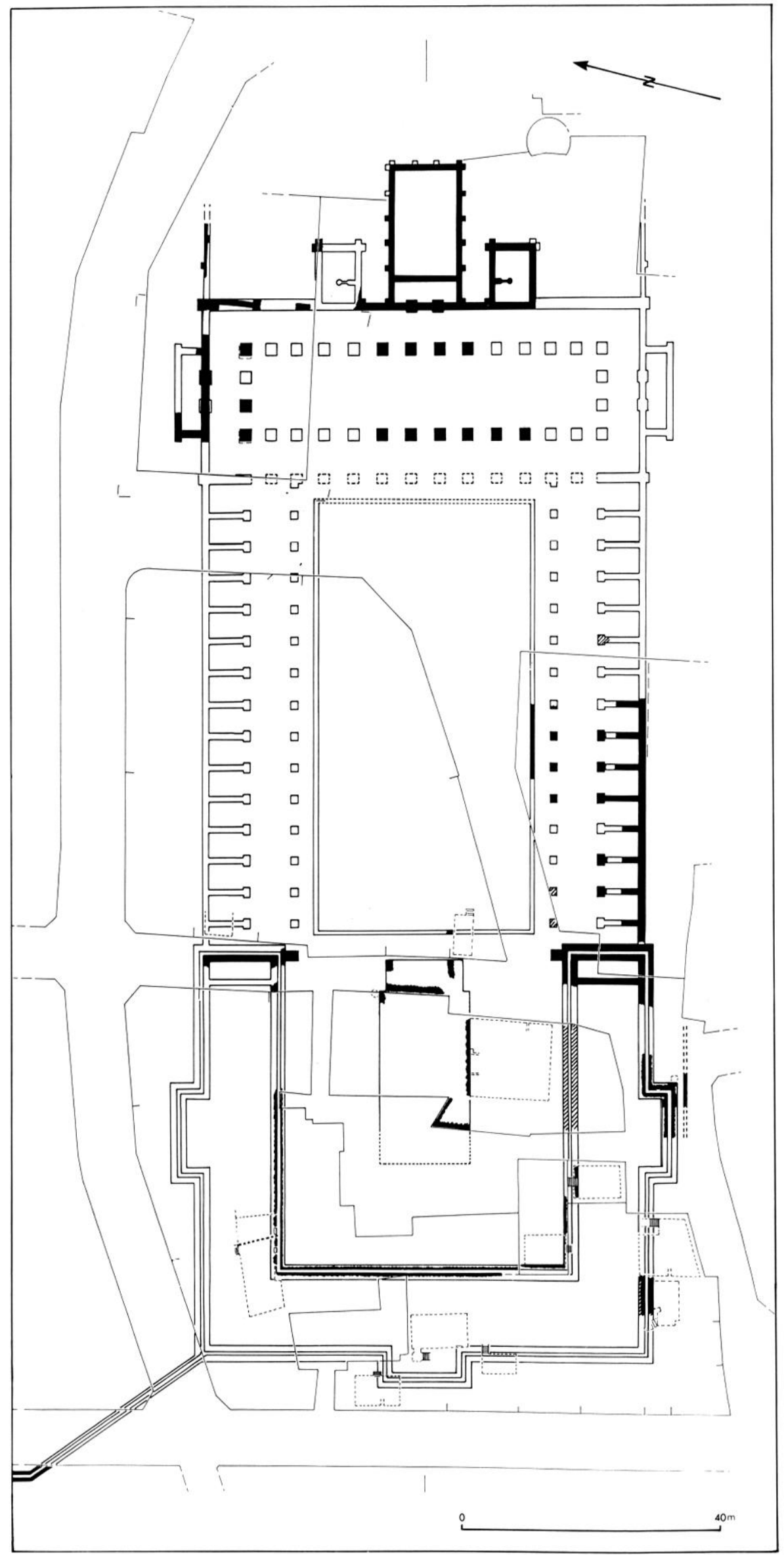

Fig. 25 - Plan restitué du forum (en noir: maçonneries précisément repérées; en hachures: maçonneries observées mais mal repérées). 
La largeur totale du forum doit donc correspondre à $5 \times 48=240$ pieds ( 2 acti), au niveau des fondations (soit 71,04 m, contre 71,1 $\pm 0,2 \mathrm{~m}$ mesurés). La longueur hors-tout de la galerie à cryptoportiques est de $59,8 \pm 0,2 \mathrm{~m}$ au niveau des fondations, soit $4 \times 48+12$ pieds. Le plan de cette partie du forum entre donc approximativement dans une trame dont l'unité est de 48 pieds, sa largeur (direction nord-sud) correspondant à cinq modules et sa longueur à quatre modules; l'aire du temple correspond à $3 \times 3$ modules.

L'aire du temple mesurée à la base de l'élévation est un carré de $45,2 \mathrm{~m}$ de côté. La longueur de chacun des trois côtés de la façade interne du cryptoportique permet de restituer une colonnade qui posséderait huit colonnes libres et deux demi-colonnes engagées à ses extrémités. Le rythme ainsi créé (entraxes de $2,85 \mathrm{~m}$, ou 9,5 pieds) est cohérent avec la position du mur de refend dégagé à l'extrémité de l'aile sud, situé de cette façon en arrière d'une colonne de la façade.

La largeur des exèdres accolées au mur d'enceinte de l'aire sacrée n'est pas connue (cf. infra, p. 157 pour la restitution de leur plan). Si la restitution d'une exèdre axiale sur la façade ouest ne fait pas de doute, on observe que celle dégagée n'est pas au milieu de la branche latérale.

Le temple lui-même a pour largeur $14,1 \mathrm{~m}$. Sa façade orientale est en retrait d'environ $5 \mathrm{~m}$ de l'extrémité du cryptoportique. Elle est précédée par un emmarchement dont l'emprise est de 12,1 $\times 4,6 \mathrm{~m}$. La longueur de la platée n'est en revanche pas connue (mais supérieure à $20 \mathrm{~m}$ ).

\section{Place centrale et portiques latéraux}

Les dimensions du portique lui-même correspondent toutes à des mesures entières en pieds (fig. 15) : entraxes des cellules de 15 pieds (valeur moyenne mesurée : $4,54 \mathrm{~m}$, contre 4,44 attendus), largeur de 48 pieds, etc. La longueur mesurée du portique $(68,2 \pm 0,2 \mathrm{~m})$ correspond assez précisément à 15 intervalles de $4,54 \mathrm{~m}(68,1 \mathrm{~m})$, ce qui permet de restituer à chacune de ses extrémités une cellule plus large, ainsi qu'on a pu le vérifier par la fouille (cf. fig. 15). La longueur totale "théorique" du portique doit donc correspondre à 15 modules de 15 pieds, soit $15 \times 15=225$ pieds. De la largeur du cryptoportique, on déduit aussi la largeur hors-tout "théorique" du forum au niveau des boutiques: $240-9=231$ pieds $(68,4 \mathrm{~m})$, qui correspond précisément à la valeur mesurée. L'aire centrale du forum s'inscrit donc exactement dans un carré de $68,4 \mathrm{~m}$ de côté.
La place elle-même a pour dimensions, si la mesure est prise au ras des piles du portique : 68,2 sur $38,4 \mathrm{~m}$, ou, si l'on suppose l'existence d'un emmarchement devant la basilique de même largeur que celui observé devant les portiques et si la mesure est prise à la base de l'emmarchement : 62,4 sur $32,4 \mathrm{~m}$, soit une proportion approximative de $2: 1$.

\section{Basilique}

Le plan de la basilique a été restitué par symétrie, en supposant que l'exèdre repérée sur sa façade septentrionale se répétait sur la façade opposée et que la largeur des portiques périphériques était uniforme.

Sa longueur hors œuvre (exèdres et contreforts non compris) est de $68,4 \pm 0,4 \mathrm{~m}$, soit exactement la largeur hors œuvre restituée du forum au niveau des portiques latéraux. Le portique se raccorde donc à la basilique de façon à ce que le mur d'appui des cellules soit dans le prolongement de sa façade.

La valeur de l'entraxe des piles de fondation de la basilique relevée sur la travée nord est de $4,1 \mathrm{~m}$ (moyenne de trois mesures), soit 14 pieds, tandis que le relevé de 1956 indique, pour les sept entraxes mesurés sur les travées est et ouest, une valeur moyenne de 4,41 $\mathrm{m}$ (avec un écart maximum de $0,17 \mathrm{~m}$ ) sensiblement plus élevée que pour la travée nord. D'autre part, la longueur totale restituée de la nef centrale entre les axes des colonnes d'ante est de $54,8 \pm 0,4 \mathrm{~m}$, soit $13 \times 4,21 \mathrm{~m}$, une valeur apparemment incompatible avec celle mesurée de $4,4 \mathrm{~m}$. L'écart est trop important pour pouvoir être mis au compte des incertitudes de relevé. On doit nécessairement supposer que l'écartement des piles des travées longitudinales n'est pas uniforme, mais plus fort vers le centre qu'aux extrémités. Il n'en reste pas moins que la nef de la basilique est limitée par quatorze colonnes sur ses longs côtés, contre quatre sur ses petits côtés.

La largeur de la nef centrale au niveau des fondations est de 14,2 $\pm 0,1 \mathrm{~m}$, soit 48 pieds (somme de quatre piles de 6 pieds de côté et de trois espaces inter-piles de 8 pieds), tandis que la longueur de nef est de $56,6 \pm 0,4 \mathrm{~m}$, ce qui correspond à $4 \times 48$ pieds $(56,8 \mathrm{~m})$, soit quatorze piles de 6 pieds et treize espaces (théoriques) de 8 pieds : le plan régulateur s'applique manifestement à l'emprise des fondations (ici l'utilisation d'un module de 48 pieds et la proportion de 4:1 de la nef centrale), comme c'était déjà le cas pour l'aire sacrée.

Qui plus est, la largeur des portiques latéraux est de $4,7 \pm 0,1 \mathrm{~m}$, soit 16 pieds et un tiers de la largeur de la nef centrale. Si nous prenons ces mèmes 
mesures dans l'axe des murs et des piliers, nous obtenons cette fois un rapport de $1: 2$, avec 21 pieds pour les portiques latéraux et 42 pieds pour la nef centrale.

Les dimensions dans wuvre "théoriques" de la basilique seraient de $(192+2 \times 16=224) \times$ $(48+2 \times 16=80)$ picds, soit respectivement de quatorze et cinq modules de 16 pieds, et done dans la proportion de $2 \sqrt{ } 2: 1$, et ses dimensions hors œuvre (sans les contreforts et les exèdres) de $231 \times 92$ pieds.

Les dimensions hors tout des édicules encadrant la curie sont sans ambiguité de 28 sur 28 pieds (en ne considérant que la partie saillante hors du mur de façade de la basilique et en incluant les contreforts) tandis que celles de la curie, mesurées de la même façon, sont de 66 sur 44 pieds, et donc dans le rapport 3:2. Sa largeur correspond approximativement à celle des exèdres appuyées sur les petits côtés de la basilique. La distance qui sépare sa façade orientale (contreforts compris) du grand axe de la basilique est de $33,3 \mathrm{~m}$ (112 pieds), soit la demi-longueur de cette dernière (dans œuvre). La basilique et la curie sont. donc nettement liées au sein du plan régulateur.

Les dimensions hors tout de l'ensemble monumental sont finalement de $173 \mathrm{~m}$ par $76 \mathrm{~m}$. L'étude de son plan montre clairement l'existence de liens organiques entre ses différentes composantes, notamment l'utilisation d'un même module de base de 48 pieds pour l'ensemble des constructions et l'identité du nombre de colonnes pour les portiques de la place centrale et la péristasis de la basilique.

\section{Niveaux de circulation}

Il s'agira essentiellement ici de rassembler les données énumérées au cours de la description. En prenant comme référence la cote de 344,0 qui correspond au niveau de circulation des portiques latéraux, la place centrale se situait entre 0,3 et $0,7 \mathrm{~m}$ en contrebas, tandis que le sol de la basilique était environ $1 \mathrm{~m}$ plus haut. On accédait de la basilique à la curie par un escalier permettant de rattraper une dénivelée supplémentaire de $1,5 \mathrm{~m}$. Du côté ouest maintenant: le sol des cryptoportiques est situé entre 2,5 et $3 \mathrm{~m}$ en contrebas. Si l'on restitue des escaliers de mème pente pour accéder du portique aux galeries inférieure et supérieure, on en déduit que le sol de cette dernière était situé entre 2,5 et $3 \mathrm{~m}$ au-dessus de celui du portique.

On ne connaît pas la cote du sommet du podium, mais il est logique de la restituer au mème niveau que la galerie supérieure des cryptoportiques. Un niveau de circulation (sol de travail) de l'aire du temple est attesté à la cote 343,6 . Comme il parait y avoir continuité des sols des portiques et de l'aire du temple, il est plausible que les deux étaient au même niveau $(344,0)$.

Le forum est établi sur un terrain qui présentait originellement une légère pente vers le nord-ouest : le sol primitif est à la cote 343,8 au sud-est, à la cote 341,6 au nord-ouest. Les niveaux de circulation gallo-romains autour du monument paraissent respecter la pente du sol primitif. Si donc on pouvait entrer de plain-pied dans le forum dans la partie est du monument, il est exclu qu'un tel accès ait pu exister sur la façade ouest, puisqu'il obligerait à couper le conduit d'assainissement. La seule entrée identifiée est sur la façade est de la basilique, et encore doit-elle correspondre à un accès secondaire. Par analogie avec les autres monuments du même type, on doit restituer d'autres entrées menant directement à la place centrale, et qui devaient se situer aux extrémités des portiques bordant cette place au nord et au sud (à travers les cellules extrêmes).

\section{LES MATÉRIAUX ET LEUR MISE EN CEUVRE; ÉLÉMENTS D'ARCHITECTURE}

\section{Les constructions en petit appareil}

Des observations précises sur le mode de construction ont pu être effectuées sur les différentes parties du complexe monumental. Il en ressort une impression d'extrême homogénéité du mode de construction, qui se double d'un certain nombre d'indices précis qui soutiennent l'hypothèse d'une construction concertée et simultanée de l'ensemble.

L'appareillage et le blocage des murs en maçonnerie utilisent presque exclusivement (à l'exception de la fondation maçonnée située en avant du portique de la place centrale) un granite rose à grain fin très dur caractéristique de la bordure nord-est de la plaine du Forez. Les carrières étaient probablement situées sur la commune de Salt-en-Donzy, sur la rive droite de la Loise, a $4 \mathrm{~km}$ de Feurs. Le mortier utilisé, blanc et très dur, à inclusions de sable grossier, paraît partout identique au seul examen visuel. Les fondations sont toujours constituées de blocage jeté en tranchées taillées aux dimensions exactes de la maçonnerie, tandis que les parties en élévation au-dessus du sol primitif montrent deux variantes :

- élévations destinées à être remblayées, qui sont toujours édifiées en petit appareil irrégulier - parement interne de la curie (fig. 26 , a), base de l'élévation des cellules des portiques, parement 

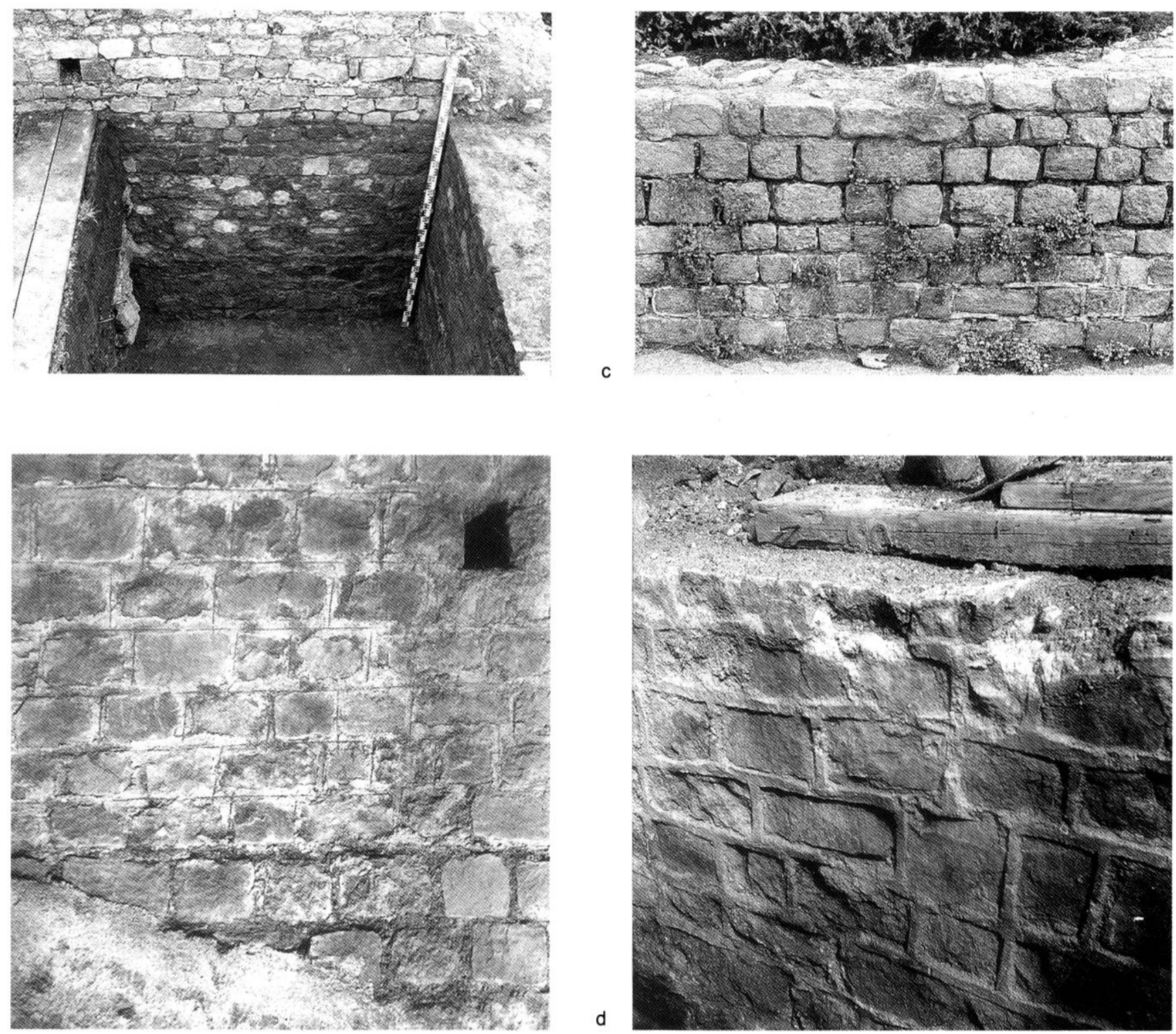

Fig. 26 - a, détail de l'appareillage interne de la curie (angle sud-est : 1988); b, détail de l'appareillage interne du cryptoportique (parement est du mur de refend, à l'extrémité de la galerie sud; 1978); c, détail de l'appareillage externe de la curie (façade sud; 1988) ; d, détail de l'appareillage interne du conduit d'assainissement du cryptoportique (extrémité de la galerie sud. parement est du tronçon nord-sud : 1978).

externe de la base de la galerie à cryptoportiques, podium du temple et parements externes de l'égout - ;

- élévations destinées à rester à l'air libre, toujours édifiées en petit appareil très régulier, mème lorsque la paroi sera ensuite enduite - parement de la galerie à cryptoportiques (fig. 26, b) - ; à l'exception de ce dernier cas. les parements présentent des joints en ruban très soignés - parements extérieurs de la curie (fig. 26, c), parement extérieur sud de la branche sud de la galerie à cryptoportiques et parements internes du conduit d'assainissement du cryptoportique (fig. 26, d) - .
On a également pu remarquer sur les élévations les mieux conservées (curie, cryptoportiques) que less murs appareillés étaient montés en séquences successives de 0,6 m de hauteur environ, qui correspondent à cinq ou six rangs de petit appareil. Les trous de boulin sont toujours situés à la base d'une nouvelle séquence. C'est une donnée intéressante sur l'organisation du travail, puisque cette façon de procéder permettait au moins d'évaluer facilement le travail effectué par chaque équipe de maçons. I)es differences dans le traitement des joints indiquent d'ailleurs que les séquences successives ont souvent été édifiées par des ouvriers différents. 
Toutes les parties du forum (cryptoportiques exceptés) ont été plus ou moins remblayées à partir du sol primitif. On possède des données précises sur le mode de constitution de ces remblais et le moment de leur mise en place dans le déroulement du chantier. Ils noient toujours la base appareillée des maçonneries, tandis que le sommet de la plupart d'entre eux (basilique, portiques, aire du temple) est constitué d'éclats de taille de calcaire jaune broyés et tassés, ce qui montre que leur installation s'est effectuée à une phase intermédiaire de la construction, avant la mise en place des élévations en grand appareil qui paraissent faire systématiquement recours à ce matériau (à l'exclusion du temple - cf. infra, p. 147). Cette constatation est confirmée par les épandages de poussière de calcaire jaune retrouvés en plusieurs points du forum (podium du temple, cryptoportiques, curie) emprisonnés dans les maçonneries au niveau des sols de travail : il semble que la totalité du monument a d'abord été entièrement édifiée jusqu'au niveau initialement prévu pour le sommet de ces remblais et que la poursuite de la construction s'est effectuée après leur mise en place, tout en laissant un délai suffisant avant la poursuite du travail pour que des salissures se déposent sur le sommet des fondations. En outre, l'analyse de la liaison de la galerie à cryptoportiques et du portique montre la simultanéité de la construction des deux ensembles (cf. supra, p. 133).

En résumé, si la durée du chantier ne peut être évaluée de façon absolue à partir des données archéologiques, il paraît probable, au vu des observations précises, mais très partielles, effectuées sur ses différentes parties, que l'ensemble du complexe a été construit d'un seul jet, voire que ses différentes parties sont sorties de terre simultanément. Cette hypothèse est également confortée par l'analyse du plan du monument, développée plus haut, qui montre qu'il a probablement été conçu intégralement en une fois.

Les seules traces de remaniement observées - encore sont-elles parfois hypothétiques - sont la surélévation des piles des portiques, dont le rang supérieur de l'appareil est très grossier, la (re)construction de l'emmarchement reliant la place centrale et les portiques, dont la facture est anormalement grossière, et enfin la réfection de l'escalier d'accès à la galerie inférieure des cryptoportiques, datée par un as de Domitien, puisque cette datation parait résolument incompatible avec celle, cohérente, issue de l'analyse des autres données stratigraphiques (cf. infra, p. 148).
Les constructions en grand appareil, les éléments ornementaux et de couverture

Les fragments d'éléments en grand appareil attribuables au forum sont très peu nombreux, sans doute à cause des récupérations dont ils ont fait l'objet à toutes les époques. La seule fouille récente de quelque envergure (1978) n'en a ainsi livré que quelques dizaines et encore de dimensions très réduites. Le matériau qui paraît avoir été le plus utilisé est un calcaire jaune à entroques, exploité dans la vallée du Sornin, à $60 \mathrm{~km}$ au nord de Feurs. En dehors du forum, ce matériau a dû être utilisé pour la construction d'un complexe monumental situé à la limite nord de l'agglomération antique, dont proviennent plusieurs fragments de fûts cannelés de $80 \mathrm{~cm}$ de diamètre environ, récupérés en 1970 lors de la construction d'un nouveau gymnase, et occasionnellement pour des éléments de statuaire, comme le montre une figuration de cavalier à l'anguipède récemment découverte sur le site (Valette, 1989). L'utilisation de ce calcaire est également attestée à Roanne, emplacement d'un vicus situé nettement plus près des carrières d'extraction, par quelques éléments d'architecture et de reliefs funéraires.

Son utilisation sur le forum semble avoir été massive si l'on en juge par l'épaisseur des épandages de déchets de taille sur les sols de travail du monument, et la relative abondance de son remploi dans des constructions postérieures, depuis l'Antiquité (par exemple dans l'appareillage d'un puits fouillé à $100 \mathrm{~m}$ au nord du forum en février 1988) jusqu'au $\mathrm{xIX}^{\mathrm{e}} \mathrm{s}$. inclus (notamment dans des façades d'immeubles situés le long de la rue de Roanne et sur la place du Puits de l'Aune - voir la liste en annexe, p. 161).

Parmi les autres matériaux figure du calcaire blanc originaire de la vallée du Rhône, qui paraît avoir été utilisé pour la construction du temple exclusivement. L'emploi de marbre n'est qu'hypothétique, en raison de la rareté des fragments retrouvés à l'emplacement même du forum.

\section{Éléments d'architecture en calcaire jaune à entroques}

L'emploi de ce matériau est d'abord attesté pour les dés supportant les colonnes de la basilique (cf. supra, p. 120), et pour des blocs de grand appareil allongé utilisés (ou réutilisés) dans l'emmarchement séparant la place centrale des portiques. Plusieurs éléments de colonnes ont également été retrouvés: 
- le premier type correspond à des fûts lisses, de taille souvent irrégulière, de diamètre compris entre 53 et $55 \mathrm{~cm}$; les faces terminales des cylindres sont en revanche soigneusement abrasées et présentent toujours un trou de louve en leur centre. L'absence de cannelures semble indiquer qu'ils étaient à l'origine stuqués. Des fragments en ont été retrouvés en divers emplacements, sur le forum et à sa périphérie : un fragment dans la fouille de 1978, dans un remblai ancien au nord du portique, un fragment dans l'appareillage d'un puits antique fouillé en 1988 à $100 \mathrm{~m}$ au nord du forum (parcelle AD131) et dont le comblement est postérieur à la fin du $\mathrm{II}^{\mathrm{e}} \mathrm{s}$. (monnaie de Marc-Aurèle), un autre dans le comblement d'un puits antique situé à $50 \mathrm{~m}$ au nord-est du forum, datable du III $^{\mathrm{e}}$ s. (Valette, 1989), enfin deux fragments réutilisés en fondation d'un bâtiment repéré en 1988 devant la façade ouest de l'église. Les colonnes peuvent appartenir au portique de la place centrale ;

- un autre élément de fût de la même fouille est de module plus important (diamètre estimé entre 75 et $80 \mathrm{~cm}$ ) et de taille très grossière, à l'exception de sa face de pose et d'un bandeau d'anathyrose de $1 \mathrm{~cm}$;

- un premier type de base de colonne est représenté par trois exemplaires dans la fouille de 1978 . Son module (diamètre de $80 \pm 8 \mathrm{~cm}$ ) est cohérent avec les fûts de $54 \mathrm{~cm}$. Tous les fragments ne présentent qu'un tore et une amorce de scotie;

- un deuxième type de base est attesté par un seul bloc, brûlé, qui conserve un ture, l'amorce de la scotie, l'ensemble étant solidaire d'une plinthe épaisse de $8 \mathrm{~cm}$ montrant sur sa face inférieure, irrégulière, l'emplacement d'un rivet scellé au plomb. Le diamètre du tore est de $\overline{5} 6 \pm 4 \mathrm{~cm}$. Ce bloc provient de l'appareillage du puits de la parcelle AD131;

- les chapiteaux ne sont représentés que par un fragment insignifiant : une feuille d'acanthe grossièrement taillée (à l'origine stuquée?), retrouvée en 1978 ;

- on doit enfin ajouter à cette liste une base de pilastre provenant des travaux de 1991. Son profil présente une doucine haute de $6 \mathrm{~cm}$ séparée par un congé d'une plinthe haute de $9 \mathrm{~cm}$.

\section{Autres matériaux}

Le calcaire blanc est seulement représenté par quelques fragments non travaillés sur la fouille de 1978, un bloc scié dans un puits fouillé en 1981 au nord-ouest du forum, une vingtaine de fragments visibles en remploi sur les façades d'immeubles sis au
6 et au 10, rue de Roanne, et deux fragments travaillés réutilisés dans le parement du puits gallo-romain de la parcelle AD131 :

- un fragment de fût cannelé, de cannelure large de $11,1 \mathrm{~cm}$ entraxes (soit un diamètre restitué de $\approx 84 \mathrm{~cm}$ ), qui appartenait à une colonne engagée, mais très en saillic de la paroi contre laquelle elle était placée;

- un fragment de fût de colonne lisse de taille assez régulière, de diamètre $66 \pm 6 \mathrm{~cm}$.

Il est très probable que le fût cannelé de grand diamètre appartenant à une colonne engagée soit attribuable au temple (ce ne pourrait ètre alors qu'une colonne adossée à la cella, indiquant ainsi que le temple est de plan pseudo-périptère, ce que suggèrent également la largeur de son podium et la hauteur de colonne que l'on est amené à restituer, qui sont toutes deux identiques aux valeurs relevées sur la Maison Carrée de Nîmes). Une tuile provenant certainement de la couverture du temple (cf. infra, p. 148) a d'ailleurs été aussi extraite du parement du puits qui a livré ce fragment d'architecture. On notera également que les remplois modernes de ce calcaire blanc n'ont été repérés que dans des façades d'immeubles situés à proximité immédiate de l'emplacement du temple. Enfin, la découverte d'un bloc mouluré restituable à la base ou au couronnement du podium à proximité immédiate de la platée, dans un remblai probablement antique, permet d'assurer définitivement que ce matériau est spécifique du temple (le bloc ne permet pas de restituer la modénature complète; n'est conservée que la séquence suivante, taillée assez grossièrement: filet, quart-derond, filet et amorce de doucine ou de cavet, le tout se développant sur une hauteur de $27 \mathrm{~cm}$ ).

De la fouille de 1978 provient un fragment de placage de colonne en marbre blanc veiné de gris, avec cannelures larges de $8,9 \mathrm{~cm}$ (d'où un diamètre de $\approx 68 \mathrm{~cm}$ ). De nombreux éléments de placage de même nature et de même module, associés à plusieurs bases aussi destinées à être plaquées sur une maçonnerie, proviennent d'un complexe monumental situé au nord de l'agglomération. Il est donc possible que l'unique fragment retrouvé à l'emplacement du forum soit un élément déplacé de même origine. Les éléments d'architecture en marbre sont aussi représentés sur la fouille de 1978 par un fragment de base de colonne en marbre blanc et trois baguettes d'applique moulurées de profils différents.

On doit enfin mentionner pour achever cette courte liste deux fragments de rigoles en granite retrouvées à l'emplacement du portique, ainsi qu'un bloc de même matière avec décor en bas relief au 
trépan représentant une feuille d'acanthe, retrouvé à l'emplacement du temple (fig. 2, point 4).

\section{Éléments de couverture}

La fouille de niveaux de destruction en place dans le cryptoportique a permis de retrouver des tuiles appartenant à la couverture de la galerie à cryptoportiques, dont les dimensions sont de $38 \times 57 \mathrm{~cm}$ (soit $2 \times 3$ modules de $19 \mathrm{~cm}$ ). De plus, le parement du puits de la parcelle AD 131 a livré une tuile de très fortes dimensions (épaisseur : $6 \mathrm{~cm}$, largeur : $45 \mathrm{~cm}$ et longueur [restituée] : $67 \mathrm{~cm}$ ) qu'il est possible d'attribuer à la couverture du temple, puisqu'un petit fragment de tuile de même épaisseur a été retrouvé dans les remblais surmontant le podium en 1984.

\section{SYNTHÈSE DES INDICES DE DATATION}

Les indications stratigraphiques utilisables pour la datation du forum ont été exposées dans les paragraphes précédents pour chaque partie du complexe monumental. Il suffira donc ici d'en faire la synthèse.

La première est fournie par les éléments les plus récents des niveaux d'occupation antérieurs au forum. Comme la dernière phase de cette occupation est très diffuse à l'emplacement du forum, il est difficile d'apprécier précisément le moment de son interruption. Les mobiliers les plus récents sont certainement postérieurs au début du principat (infimes fragments de sigillée italique, gobclets à parois fines de type Aco, fibule à charnière, lèvre d'amphore Dr. 7/11), mais parmi ces objets aucun ne permet de suggérer que cette occupation s'est prolongée au-delà du règne d'Auguste. Le seul ensemble clos de mobilier qui apporte des indications précises pour l'emplacement du forum proprement dit est le puits fouillé en 1978 sous le portique sud. Son comblement est en tout état de cause datable de la fin du I $^{\text {er }}$ s. avant J.-C. ou du début du $I^{e r} s$. après J.-C. Les niveaux contemporains de la construction du monument n'ont livré aucun élément datable à l'exception d'un as de Domitien pris dans une couche contemporaine de la (re)construction de l'escalier de descente à la galerie à cryptoportiques.

Des données précises sont enfin fournies par les couches retrouvées en relation stratigraphique avec l'égout du forum et qui permettent sans ambiguïté possible de dater sa construction aux alentours de 10 après J.-C. Même si la mise en chantier de cet égout doit logiquement se placer au début des travaux d'édification du forum, il ne semble pas que l'on puisse beaucoup différer la date de construction de l'ensemble du complexe monumental, à cause de l'homogénéité de son mode de construction et d'un argument a silentio non négligeable : l'absence de tout vestige gallo-romain postérieur à la fin du règne d'Auguste dans les niveaux d'occupation précoces dégagés à son emplacement, fait qu'il serait difficile de concevoir si l'cmplacement du forum était resté libre au-delà de cette époque puisqu'il est situé au cœur de la ville antique et que l'implantation d'un habitat gallo-romain structuré est attestée à sa proximité immédiate dès la deuxième décennie de notre ère (Vaginay, Valette, 1982). On notera d'ailleurs que des petits fragments du calcaire jaune importé pour la construction du forum ont été retrouvés dans les niveaux les plus anciens de ce quartier d'habitat. La seule "fausse note" dans ce tableau reste donc la monnaie de Domitien dont la situation stratigraphique ne peut être expliquée que par une réfection de l'escalier d'accès au cryptoportique.

L'époque du démantèlement du monument n'est en revanche pas situable précisément. On sait que la galerie à cryptoportiques a été définitivement abandonnée à la suite d'un incendie, dont des traces de coup de feu sur plusieurs blocs de grand appareil (blocs en place sur le mur de la curie, blocs en remploi dans le puits de la parcelle AD131) semblent également témoigner. Le niveau d'incendie de la galerie ne recelait que quelques tessons, dont les plus caractéristiques ne paraissent pas pouvoir être datés avant la fin du ${ }_{1}{ }^{\circ} \mathrm{s}$. Des indications analogues sont fournies par les deux puits dans lesquels ont été retrouvés des éléments d'architecture attribuables au forum : comblement datable du in ${ }^{\mathrm{e}}$ s., au moins pour l'un d'entre eux (Valette, 1989). On doit également rattacher à cette époque un fragment d'autel $(C I L$ XIII, 1643) provenant probablement du forum, autrefois en remploi dans une remise située à son emplacement. L'inscription, réduite à une ligne, est restituée : OTACILIA SE[VERA], sans doute Marcia Otacilia Severa, femme de Philippus Senior, empereur de 244 à '249.

En l'absence d'indice suggérant un prolongement de l'utilisation du forum au-delà du ur' s., l'hypothèse la plus plausible - mais non prouvée - est celle d'un démantèlement lié aux invasions du troisième quart du $11{ }^{\circ}$ s. Cette proposition est également cohérente avec les observations faites récemment sur divers quartiers d'habitat de la ville antique.

\section{L'INTÉGRATION DU FORUM DANS LA TRAME URBAINE}

Le forum est situé au cœur de l'agglomération antique. Dans l'état actuel de nos connaissances, 
l'emprise de la ville a la forme d'une bande allongée le long du cours de la loire dont la superficie est approximativement de 70 ha. I.e centre se situerait à l'emplacement du forum qui occupe une position privilégiée, au point de rencontre des rebords des vallées alluviales du fleuve et d'un affluent, la I,oise (fig. 27). Vers le sud, la ville s'étend largement sur le replat délimité par ces deux vallées, jusqu'à plus de $500 \mathrm{~m}$ du forum, tandis que vers le nord elle a colonisé les deux versants de la Loise. Un complexe monumental, hâtivement repéré lors de travaux d'urbanisme, faisait face au forum, sur le rebord septentrional du vallon. Le théâtre, connu seulement par son inscription dédicatoire retrouvée en 1887 à $150 \mathrm{~m}$ au nord-ouest du forum, était certainement adossé à l'un des versants du même vallon.

La ville était traversée par deux axes de circulation principaux : dans la direction est-ouest, par la voie d'Aquitaine qui, venant de Lyon et se dirigeant vers Clermont-Ferrand, abordait Feurs en suivant la rive gauche de la Loise avant de franchir la Loire (sans que l'on sache où se situait le point de traversée du fleuve), et dans la direction nord-sud par un axe secondaire qui longeait la rive droite du fleuve. Si l'on ignore encore le tracé exact de ces routes aux abords de la ville, l'organisation de la voirie urbaine, seulement connue jusqu'à ces dernières années par quelques observations de Roux reportées sur un plan très imprécis (Roux, 1851, plan h.-t.), a en revanche été largement précisée au cours de la dernière décennie (fig. 28). Une trame orthogonale, cohérente avec l'orientation du forum, recouvrait la majeure partie de l'agglomération: les seuls vestiges de construction dégagés qui ne respectent pas son alignement sont localisés, soit au-delà de la Loise (̣point 2), soit à plus de $600 \mathrm{~m}$ au sud-ouest du monument (point 14). L'absence d'accident topographique contraignant explique sûrement à lui seul la simplicité de cette trame, qu'il suffisait de prolonger au gré de l'extension de la ville sans avoir à fléchir son orientation. A la précision des relevés, les cinq rues qui ont été identifiées respectent donc l'alignement du forum, dont l'axe longitudinal, exactement repéré. nous a servi à restituer une partie de la trame urbaine.

Tous les vestiges antiques ont été reportés sur un plan d'ensemble de l'agglomération au 1/2000'. Leur repérage a été effectué à partir de leur localisation sur le parcellaire actuel, pour lequel on dispose de relevés géodésiques récents, avec l'intégration au carroyage Lambert.

Des vestiges de voirie ont été localisés en quatre emplacements. Ils permettent de tracer trois rues nord-sud et deux rues est-ouest.

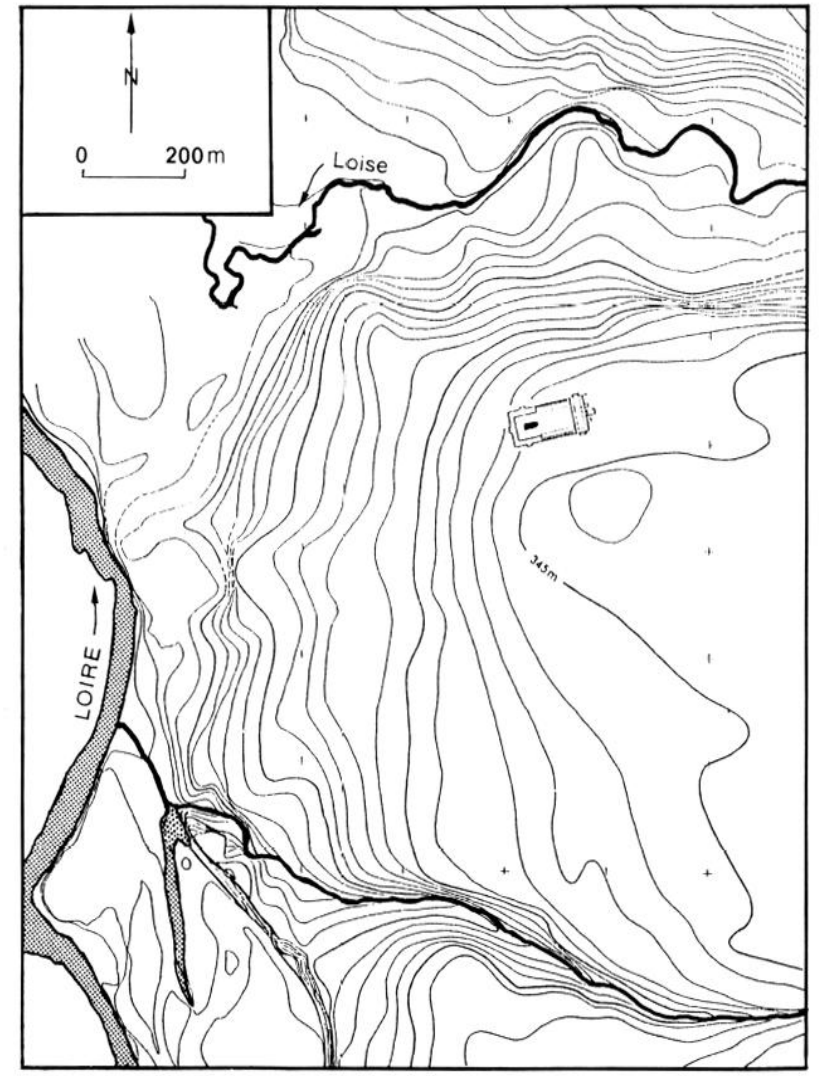

Fig. 27 - Topographie du site de Feurs (équidistance des courbes de niveaux : $1 \mathrm{~m}$ ).

Les rues nord-sud ont été indexées I, II et IV. Leurs distances entraxes sont :

- entre I et II : $108 \pm 2 \mathrm{~m}$,

- entre II et IV : $214 \pm 2 \mathrm{~m}$.

L'écartement des rues I et 11 est donc très proche de la valeur attendue pour trois acti (360 pieds, soit $106,5 \mathrm{~m}$ ). Celui des rues II et IV permet aussi de restituer exactement une rue intermédiaire III respectant le même rythme. Des vestiges repérés dans de mauvaises conditions en 1988 (fig. 28, point 9 : épandage de cailloutis bordé de constructions) correspondent probablement à une rue supplémentaire $\mathrm{V}$ également distante de la précédente de trois acti.

Les rues est-ouest repérées ont été indexées $\Lambda$ et D. La distance entre la rue $A$ et le grand axe du forum est encore de $108 \pm 2 \mathrm{~m}$, ce qui laisse supposer qu'une rue $B$ parallèle à $A$ est située dans cet axe (on sait par ailleurs, avec la fouille au point 7, qu'aucune rue ne longe les longs côtés du forum). En supposant aussi l'existence d'une rue $C$ symétrique de $A$ par rapport à l'axe du forum (également suggérée par le plan de Roux, qui figure un tronçon assez précisément là où il est attendu), l'emprise du monument apparaît répartie sur quatre îlots. Cette disposition 


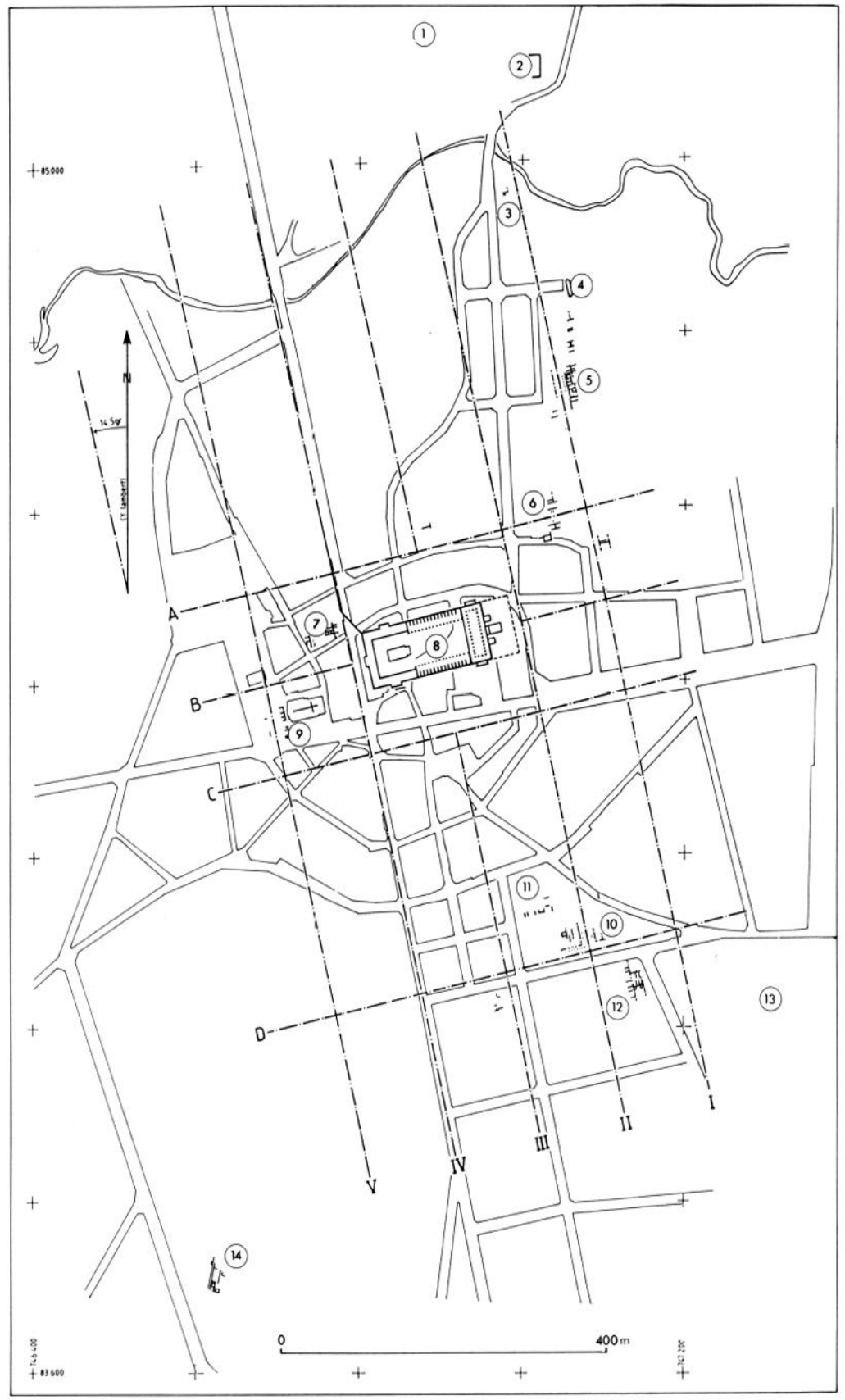

Fig. 28 - Carte archéologique de Feurs (état fin 1990), avec une tentative de restitution partielle de la trame urbaine antique.

1: complexe monumental non-identifié (observations anciennes et lors de travaux d'urbanisme en 1970);

2: monument non-identifié (péribole de fanum ? Fouille de J. Gorce, 1960);

3 : murs antiques en élévation (thermes?);

4 : bassin à exèdres (villa suburbaine? Fouille de 1985);

5 : rue nord-sud bordée d'immeubles à portiques (fouille de 1985-1986);

6 : rue est-ouest bordée d'immeubles à portiques, mosaïque (fouille de 1985);

7 : égout du forum, rue nord-sud, habitat gaulois et gallo-romain (fouille de 1981);

8 : habitat gaulois, forum (fouilles de 1955 , 1968, 1978-1980);

$9:$ rue nord-sud (?) bordée de constructions (repérage de 1988);

10 : nécropole gauloise, carrefour de rues bordées d'immeubles à portiques (fouille de 1984);

11 : quartier d'habitation (sondages de 1990); 12: quartier d'habitation (fouille de 1987);

13: monument non-identifié (mausolée? Indication de Roux);

14 : mur à exèdre et bassin (villa suburbaine? Fouille de 1988). ménage sur toutes ses faces de larges espaces qui n'ont pas été explorés. On remarque en particulier que l'espace libre sur la façade orientale permettrait de restituer un enclos entourant la curie de proportions approximatives de 1:2.

La distance entre l'hypothétique rue $\mathrm{C}$ et la rue D est de $284 \pm 2 \mathrm{~m}$, soit huit acti, ce qui ne permet plus de restituer un rythme uniforme de trois acti dans cette direction. On peut alors envisager un découpage en trois modules de 320 pieds ou en quatre modules de 240 pieds. En fait, une campagne de sondages du printemps 1990 (fig. 28, point 11) a fourni des résultats qui s'opposent à la dernière pos- sibilité, en l'occurrence des vestiges d'habitat dans l'axe d'une rue attendue.

Le forum apparaît donc comme le pivot de la trame urbaine, ce qui n'est pas sans incidence sur son histoire et son architecture. Il est d'abord certain que l'espace qui lui était destiné a été réservé dès la mise en place de la voirie, soit au plus tard vers la fin du règne d'Auguste : au cas contraire on aurait en effet retrouvé des vestiges de rues recouverts par le forum à l'emplacement de la curie (rue est-ouest B située dans l'axe longitudinal du forum) et du portique sud de la place centrale (rue nord-sud III). Du point de vue architectural, on remarquera que la res- 
titution d'une rue axiale incite à penser que le centre des façades correspondantes (façade occidentale du cryptoportique et façade orientale de la curie) bénéficiaient d'un traitement particulièrement soigné. Le cas se rencontre à Trèves (dont la trame urbaine primitive présente d'ailleurs de fortes analogies avec celle de Feurs) : la façade occidentale du cryptoportique est dans cet exemple muni d'une exèdre, qui a certainement aussi existé à Feurs. Du point de vue fonctionnel, on peut suggérer que les espaces libres latéraux étaient des emplacements commerciaux ${ }^{18}$.

\section{ANALYSE ARCHITECTURALE ET ETUDE COMPARATIVE}

Nous retiendrons deux faits majeurs : le premier est que le forum de Feurs vient s'ajouter à la liste des fora de conception tripartite, associant en un ensemble cohérent aire sacrée, espace public et groupe basilique-curie. Le second est que la datation archéologique de son édification et l'absence de remaniements majeurs affectant son organisation laissent supposer que ce schéma était tout à fait fixé en Gaule dès le début du I $^{\mathrm{er}} \mathrm{s}$.

Il n'est pas de notre propos de reprendre ici l'immense dossier des fora impériaux. Nous utiliserons les éléments de comparaison dans une perspective privilégiant la chronologie, afin de confronter la datation que nous proposons pour Feurs à celles disponibles dans d'autres cas.

Nous considérerons d'abord le plan dans sa globalité, en négligeant la spécificité architecturale de telle ou telle partie. L'étude de la basilique et de l'aire sacrée, en particulier, sera reprise ensuite.

\section{UN FORUM TRIPARTITE}

Il convient tout d'abord de préciser les caractères qui, parmi une documentation abondante, permettent d'isoler les monuments utilisables comme références. Pour cela, la notion de forum tripartite, fréquemment utilisée, doit être rappelée.

Ce terme désigne la traduction architecturale des fonctions du forum: la fonction politico-religieuse et la fonction civile (essentiellement judiciaire), les deux étant d'ailleurs difficilement dissociables. Les travaux de J.-M. David ont montré

18 Le macellum est souvent situé à proximité immédiate du forum (exemples à Ordona, Clunia, Belo, Nyon, Pompéi, Ampurias); les cellules extérieures accolées au mur de clôture (comme à Reims, Bavay, Paris et Trèves) peuvent d'ailleurs correspondre à l'intégration de cette fonction au projet architectural du forum. comment la basilique va, à partir de l'époque d'Auguste, acquérir un rôle prééminent par l'adjonction d'une ædes Augusti, "espace supplémentaire introduisant la présence d'un autre pouvoir politique qui s'ajoutait à celui du magistrat, et souvent le dominait" (David, 1983, p. 228). Aussi la présence d'un tcmple du culte impérial n'est-elle pas nécessaire sur un forum. Il est possible de citer ceux de Ruscino (Barruol, Nickels, 1980; essai de restitution de l'élévation par Barruol, Marichal, 1987), peut-être celui d'Arles dans sa phase augustéenne (Gros, 1987, p. 347) ${ }^{19}$ et aussi, selon la nouvelle lecture qui vient d'en être faite, celui de Conimbriga, également augustéen (Roth-Congès, 1987b). Il en est de même à Doclea, en Yougoslavie (Sticotti, 1913, p. 106-138). Ce phénomène se retrouve, pour une époque plus tardive, dans les fora britanniques (Silchester, Cærwent), au plan plus proche de celui des castra que des modèles italiques. A Martigny, où les deux états du forum sont datés de Claude et de Vespasien (Wiblé, 1981), un temple existe, mais à sa périphérie. D'autre part, des fora de schéma tripartite pourraient être dépourvus de temple: Bavay, Trèves.

Il est bien évident que la présence d'un temple revêt une signification idéologique toute particulière. Au forum d'Arles, où se dressait l'autel au Genius Augusti et aux Cæsares, est adjoint dans le premier tiers du $\mathrm{I}^{\mathrm{er}} \mathrm{s}$. un ensemble monumental imité du forum d'Auguste (Gros, 1987, p. 357-360).

En revanche, il est difficile de concevoir un forum sans basilique à l'époque impériale ${ }^{20}$. Le début de cette période est caractérisé par un fort développement de ce type de construction. Pour l'Italie, H. Jouffroy (Jouffroy, 1986, p. 48-49, 90-91) a recensé dix-neuf basiliques républicaines, attestées par l'épigraphie ou l'archéologie. Douze nouvelles seront édifiées (en particulier dans les villes du Nord) sous les Julio-Claudiens et quatre seulement sous les Flaviens. Hors d'Italie, la basilique absente sur le forum républicain d'Ampurias sera construite à l'époque augustéenne. Il faut également citer le cas de Zadar, où la basilique est une addition plus tardive sur une des branches du portique.

Deux critères principaux permettent de classer les différentes dispositions possibles de l'espace à l'intérieur du forum.

19 Le centre de la place pouvait ètre occupé par «un autel assorti probablement d'un enclos et peut-ètre d'un baldaquin ".

20 A ce propos, se reporter aux remarques d'Anne Roth-Congès au sujet du forum de Conimbriga dans son troisième et dernier aménagement (Roth-Congès, 1987b, p. 728 , note 65 ). 
L'un est souligné depuis longtemps : c'est l'axialité qui peut exister entre l'aire sacrée et la basilique, ce qui suppose que cette dernière soit implantée sur le petit côté opposé au temple, l'autre solution consistant à l'édifier sur l'un des grands côtés (cf. par exemple: Ward-Perkins, 1970, p. 7 ; Jimenez-Salvador, 1987 a el b). Les exemples du second type sont nombreux: citons ceux de Virunum, d'Ampurias (phase augustéenne), Sagonte, Bilbilis, Luni, Conimbriga. Néanmoins, ce sont ceux du premier type qui semblent avoir prévalu dans les provinces occidentales ${ }^{21}$. Selon Ward-Perkins, la diffusion s'en serait faite à partir des prototypes augustéens d'Italie : Benevagienna, Velleia, Zuglio ..., euxmêmes redevables d'ensembles plus anciens comme celui d'Alba Fucens, daté de la première moitié du $\mathrm{I}^{\mathrm{er}} \mathrm{s}$. avant J.-C. Une synthèse plus récente (Gros, Torelli, 1988, p. 344-345), s'appuyant sur les résultats des dernières recherches sur les fora augustéens de Narbonnaise, d'Afrique et de la péninsule ibérique, remet en cause ce schéma. Elle insiste sur la durée et la variété des expérimentations, qui touchent en particulier la structure de la basilique et de l'aire sacrée et la hiérarchisation des espaces. Sur le plan chronologique, les deux modèles coexistent dès le début de l'Empire. Les cas de Doclea et de Ruscino, où la place entourée de portiques a une forme proche du carré et où le temple n'existe pas, sont plus difficilement classables.

L'autre critère est celui de l'affirmation plus ou moins prononcée de l'aire sacrée à l'intérieur du schéma d'ensemble. Celle-ci se fait généralement par la présence d'une porlicus triplex, parfois sur cryptoportiques, encadrant le sanctuaire. Mais on assiste le plus souvent à une véritable individualisation par rapport à la place bordée de portiques qui constitue le forum proprement dit. Entre les deux, il peut exister un axe de circulation, comme à Augst (LaurBelart, 1966), Benevagienna, Luni (CavalieriManasse el alii, 1982, p. 157-161) ou Minturnes (Coarelli, 1982, p. 372-375), une clôture, comme à Assise, où la limite de l'aire sacrée est un mur orné de guirlandes et de rinceaux en bronze (Gros, Theodorescu, 1985), ou les deux à la fois, comme à Nyon (BonnardYersin et alii, 1989, p. 12). L'indépendance des deux espaces est formulée encore plus explicitement à Saint-Bertrand-de-Comminges, où le temple tourne le dos à la place. Les exemples a contrario de Zuglio

21 A l'exclusion de l'Afrique, où le forum tripartite est. très rare. Ie seul exemple retenu par G. IIallier (IIallier 1984) est le premier forum de Rougga, daté de la seconde moitié du $\mathrm{I}^{\mathrm{er}} \mathrm{s}$., encore ne correspond-il pas au modèle "canonique". et Clunia, où ce phénomène n'existe pas, prouvent qu'il n'est pas lié obligatoirement au type du forum tripartite. Il convient donc de ne pas "rigidifier» ces schémas et de tenir compte des circonstances dans lesquelles les ensembles monumentaux ont été mis en place : contraintes dues à la topographie (Bilbilis, Manigua, Ordona, Glanum), à l'existence d'édifices plus anciens, ou au développement de programmes architecturaux successifs (Conimbriga). Le remploi d'éléments antérieurs rend parfois extrêmement complexe la lecture des vestiges et ce n'est pas le moindre avantage du forum de Feurs de permettre l'économie de ces difficultés.

En Gaule, le plan tripartite a connu une faveur certaine. Aux côtés des exemples les plus fréquemment cités de Paris, Bavay, Saint-Bertrand-deComminges, Alésia et Trèves, il faut ajouter ceux de Limoges (Loustaud, 1981), Périgueux (Doreau et alii, 1985 ) et $\Lambda$ miens (Bayard, Massy, 1983). Les vestiges découverts à Metz (Lefèbvre, Wagner, 1984; Vigneron, 1986), à Poitiers (Gallia, 33, 1975, p. 382-383 et 35, 1977, p. 383-384), à Aix-en-Provence (Gallia, 44, 1986, p. 380, fig. 5; Rivet, 1987) et Avignon (Salviat, 1979; Gallia, 42, 1984, p. 407-411) sont en revanche encore trop ténus pour pouvoir être pris en compte.

Cette documentation potentielle offre de nombreuses lacunes que les travaux actuels tentent de combler, le plus souvent à partir de la reprise de fouilles anciennes ou seulement d'une nouvelle lecture de leurs résultats. Ils permettent de préciser certains plans, comme celui du forum de Paris (Gallia, 33, 1975, p. 319-322; Périn, 1984) ou encore du forum d'Avenches (Bossert, Fuchs, 1989), d'affiner la chronologie, par exemple à Alésia (Gallia, 41, 1983, p. 395 ; Mangin, Bénard, 1985), à Saint-Bertrand-deComminges (Gallia, 44, 1986, p. 316-318; Sablayrolles, Sillières, 1987) ou à Bavay (Hanoune, Muller, 1988). Ces recherches conduisent parfois à une vision tout à fait nouvelle des monuments, comme en témoignent la mise en évidence des deux états principaux du forum de Glanum (Roth-Congès, 1987a) ou la nouvelle interprétation des vestiges de Conimbriga (Roth-Congès, 1987b).

La datation qui a longtemps prévalu pour la diffusion du forum tripartite en Gaule correspond à la fin du I $^{e r} \mathrm{~s}$. et au $\mathbf{I}^{\mathrm{e}} \mathrm{s}$. A défaut de données stratigraphiques suffisantes, ce dont rendent compte les pages déjà anciennes de Grenier (1958), on s'appuyait sur des arguments épigraphiques (temple d'Augst), des particularités du mode de construction (parfois elles-mêmes mal datées) telles l'usage de cordons de briques (basilique d'Augst). A cela s'ajoutait la référence à certains monuments de l'lirbs, au 
forum de Trajan en particulier, considérés comme des modèles obligés de l'architecture provinciale (Crema, 1959, p. 364; Ward-Perkins, 1970, p. 7). La datation flavienne proposée plus récemment pour la seconde phase du forum de Conimbriga renforçait ce point de vue (Alarção, Étienne, 1977).

Les données précises sur les fora de Gaule interne, en particulier celles relatives à leur datation, sont effectivement très peu nombreuses ${ }^{22}$ et l'on conçoit que les études récentes abordant le sujet soit préfèrent retenir des datations vers la fin du $\mathrm{I}^{\mathrm{er}} \mathrm{s}$. ou le début du ${ }_{1 I^{\mathrm{e}}} \mathrm{s}$. (Gros, Torelli, 1988, p. 345, 351), soit se montrent prudentes en matière de chronologie (Goudineau, 1980, p. 274), soit plus simplement se bornent à une nomenclature des édifices, reconnaissant que "toute synthèse serait risquée" (Bedon et alii, 1988, p. 229).

Dans l'état actuel de la recherche, la construction du forum de Feurs au début du I $^{\mathrm{er}} \mathrm{s}$. apparaîtrait donc particulièrement précoce pour la Gaule interne. Les données extérieures à la Lyonnaise montrent cependant clairement que le schéma tripartite s'est développé dès les premières décennies de l'Empire. C'est le cas, en Italie, des fora de Benevagienna, dont la construction est mise en rapport avec laccession de la ville au droit latin en $5 / 4$ avant J.-C., de Velleia, daté de la période augusto-tibérienne, ou encore de Zuglio, de la première moitié du $\mathrm{I}^{\mathrm{er}}$ s. après J .-C. De même, en Narbonnaise, le forum de Glanum, édifié vers 20 avant J.-C., exprime déjà un schéma tripartite (temples avec péribole, portiques et basilique) adapté à la fois à la topographie et aux traditions religieuses indigènes (Roth-Congès, 1987a, p. 197). Le forum de Ruscino, déjà en place en 8 après J.-C. d'après les données épigraphiques (Barruol, Nickels, 1980), montre également une organisation cohérente, intégrant basilique, portiques et æ2os Augusti. A Saint-Bertrand-de-Comminges, une datalion augustéenne du forum, mise en relation avec l'acquisition du droit latin, est confirmée par la stratigraphie, du moins pour la zone du temple (Sablayrolles, Sillières, 1987).

Le forum de Nyon, en Germanie supérieure, dont le plan est très proche de celui de Feurs, comporte plusieurs états que révèle l'étude de ses

22 Notons la remise en cause prudente de la datation du forum d'Amiens, en faveur d'une date plus haute : la datation de la zone orientale (macellum?), assurée stratigraphiquement $(60-80 / 90)$, est étendue à la zone occidentale du fait de l'absence de constructions antérieures dans les insulæ quelle occupe, et que celles-ci ont joué "un ròle privilégié dès la création du grand quadrillage urbain" (Bayard, Massy, 1983, p. 8.3). basiliques (Kaenel, Tuor, 1978). Il se met en place depuis l'époque tardo-augustéenne (première basilique, à deux nefs) jusqu'aux époques tibéro-claudienne (cryptoportiques) et néro-flavienne (second état de la basilique). A Avenches (Bossert, Fuchs, 1989 , p. 38), quatre états du forum ont été déterminés à partir des données de fouilles anciennes. Le plan tripartite y émerge dans le deuxième état daté de l'époque claudienne ou néronienne.

Les provinces ibériques ont connu, elles aussi, une large diffusion du schéma tripartite. J.-L. Jimenez-Salvador (1987a et b) souligne les difficultés à en établir une synthèse dans la mesure où les études exhaustives sont récentes (Ampurias, Bilbilis, Belo). Cependant, sans vouloir multiplier les exemples, il apparaît nettement que le schéma tripartite se généralise en Espagne dans la première moitié du $\mathrm{I}^{\mathrm{er}} \mathrm{s}$. Ainsi, le plan du forum de Feurs peut être plus particulièrement rapproché de celui de Clunia ${ }^{23}$ et, à un degré moindre, compte tenu de ses proportions, de celui de Belo, deux exemples "bien datés". Le premier présente une grande régularité et une homogénéité qui peuvent laisser supposer, comme à Feurs, la réalisation d'un seul programme de construction. Celle-ci serait de peu postérieure à la fondation tibérienne de la ville. Le second, objet d'une étude approfondie depuis $1967^{24}$, est l'un des mieux connus de la péninsule. Établi sur des vestiges antérieurs encore peu explorés, son réaménagement monumental remonte à l'époque de Claude ou de Néron.

\section{L'ENSEMBLE BASILIQUE-CURIE}

Il n'est pas de notre propos, dans cette étude comparative, d'entrer dans les multiples problèmes que pose l'édifice basilical dans l'architecture antique, d'autant plus que l'absence de découvertes de fragments d'architecture ${ }^{25}$ pouvant se rapporter à la basilique ne permet pas de régler définitivement le problème de la colonne, rien ne permettant de déduire ses dimensions de la valeur de l'entraxe. La dimension des hypobases $(1,2 \times 1,2 \mathrm{~m})$ fixe néanmoins la limite supérieure de leur diamètre. (1987).

23 Cette parenté a déjà été soulignée par De Palol

24 Publication régulière des campagnes de fouilles dans les Mélanges de la Casa Velasquez depuis cette date.

25 Pour expliquer ce fait, faut-il supposer. comme a Ruscino (Barruol, Marichal, 1987, p. 5), lexistence de colonnes en blocage recouvertes d'enduit? La technique du stucage est attestée archéologiquement sur les colonnes des basiliques de Pompéi, Sagonte (Aranegui et alii. 1987. p. 89) et Belo. 
Nous avons retenu, parmi les nombreux exemples existants, et pas toujours bien connus, ceux dont le plan présente une analogie avec celui de Feurs. Pour chacun d'eux, nous avons rassemblé un certain nombre de données métriques, ainsi que le nombre de colonnes de soutènement de la nef centrale (tabl. I). L'intervalle chronologique est large, depuis la basilique de Pompéi (fin du $\mathrm{II}^{\mathrm{e}} \mathrm{s}$. avant
J.-C.) jusqu'à celle de Trieste (seconde moitiẻ du II $^{\mathrm{e}} \mathrm{s}$. après $\mathrm{J}$.-C. pour le second état).

On constate d'abord l'absence d'évolution technologique évidente, puisque la largeur de la nef centrale de la basilique de Feurs, par exemple, n'est pas plus importante que dans l'exemple de Pompéi. Qui plus esl, une portée neltement plus élevée est attestée au début de l'époque augustéenne dans l'édifice

Tableau. I - Dimensions comparées de quelques basiliques de forum

(dimensions générales : dans œuvre, en ne prenant en compte que la nef centrale et ses déambulatoires; largeur de la nef centrale : entre les axes).

\begin{tabular}{|c|c|c|c|c|c|c|c|}
\hline Lieu & Dimensions & Péristasis & $\begin{array}{c}\text { Largeur } \\
\text { nef centrale }\end{array}$ & $\begin{array}{c}\text { Rythme } \\
\text { colonnade }\end{array}$ & $\begin{array}{l}\text { Diamètre } \\
\text { colonnes }\end{array}$ & Datation & Bibliographie \\
\hline Pompéi ......... & $53 \times 25 \mathrm{~m}$ & $4 \times 12$ & $12 \mathrm{~m}$ & $3,8 \mathrm{~m}$ & $1,1 \mathrm{~m}$ & $\begin{array}{l}\text { fin } I^{\mathrm{e}} \text { s. avant } \\
\text { J.-C. }\end{array}$ & Ohr, 1977, p. 19 \\
\hline Fano $\ldots \ldots \ldots \ldots$ & $160 \times 100$ pieds & $4 \times 8$ & 60 pieds & 20 pieds & 5 pieds & 40/20 avant J.-C. & Vitruve, V, 1 \\
\hline Corinthe $\ldots \ldots \ldots$ & $46 \times 22 \mathrm{~m}$ & $4 \times 11$ & $12,3 \mathrm{~m}$ & & & $\begin{array}{l}\text { dernier quart } \mathrm{I}^{\mathrm{er}} \mathrm{s} \text {. } \\
\text { avant J.-C. }\end{array}$ & Balty, 1962 \\
\hline Ruscino .......... & $48 \times 19 \mathrm{~m}$ & $4 \times 8$ & $9,6 \mathrm{~m}$ & $4,9 / 3,2 \mathrm{~m}$ & $0,9 \mathrm{~m}$ & $\begin{array}{l}\text { époque } \\
\text { augustéenne }\end{array}$ & $\begin{array}{l}\text { Barruol et alii, } \\
1980\end{array}$ \\
\hline Ordona $\ldots \ldots \ldots$ & $42 \times 27 \mathrm{~m}$ & $4 \times 8$ & $15,9 \mathrm{~m}$ & $5 \mathrm{~m}$ & & $\begin{array}{l}\text { époque } \\
\text { augustéenne }\end{array}$ & Mertens, 1973 \\
\hline Tarragone ....... & $75 \times 28 \mathrm{~m}$ & $4 \times 14$ & $14,2 \mathrm{~m}$ & $4,8 \mathrm{~m}$ & $0,9 \mathrm{~m}$ & $\begin{array}{l}\text { époque } \\
\text { augustéenne (?) }\end{array}$ & $\begin{array}{l}\text { Mar, Ruiz de } \\
\text { Arbulo, } 1987\end{array}$ \\
\hline Feurs $\ldots \ldots \ldots$ & $69 \times 24 \mathrm{~m}$ & $4 \times 14$ & $12,3 \mathrm{~m}$ & $4,2 \mathrm{~m}$ & & $\begin{array}{l}\text { début du } \mathrm{I}^{\mathrm{er}} \mathrm{s} \text {. } \\
\text { après J.-C. }\end{array}$ & \\
\hline Périgueux $\ldots \ldots$. & $85 \times 25 \mathrm{~m}$ & $4 \times 12$ & $13 \mathrm{~m}$ & & & $\begin{array}{l}\text { époque julio- } \\
\text { claudienne? }\end{array}$ & $\begin{array}{l}\text { Doreau et alii, } \\
1985\end{array}$ \\
\hline Glanum ( $2^{\mathrm{e}}$ état) .. & $46 \times 22 \mathrm{~m}$ & $4 \times 10$ & $11,8 \mathrm{~m}$ & $3,9 \mathrm{~m}$ & & $\begin{array}{l}\text { époque julio- } \\
\text { claudienne }\end{array}$ & $\begin{array}{l}\text { Gros, Varène, } \\
1984\end{array}$ \\
\hline Trieste (1 ${ }^{\text {er }}$ état) .. & $60 \times 23 \mathrm{~m}$ & $4 \times 12$ & $12 \mathrm{~m}$ & & & $\begin{array}{l}\text { époque julio- } \\
\text { claudienne }\end{array}$ & Zaccaria, 1988 \\
\hline Clunia .......... & $81 \times 26 \mathrm{~m}$ & $4 \times 14$ & $12,7 \mathrm{~m}$ & & & époque claudienne & De Palol, 1987 \\
\hline Belo $\ldots \ldots \ldots \ldots$ & $37 \times 20 \mathrm{~m}$ & $4 \times 8$ & $9,1 \mathrm{~m}$ & & & époque néronienne & $\begin{array}{l}\text { Mémoires de la } \\
\text { Casa Vélasquez, } \\
\text { IX, } 1973\end{array}$ \\
\hline Nyon (2e état) .... & $53 \times 22 \mathrm{~m}$ & stylobate & $12,0 \mathrm{~m}$ & & & époque néronienne & $\begin{array}{l}\text { Kaenel, } \\
\text { Tuor, } 1978\end{array}$ \\
\hline Trèves $\ldots \ldots \ldots$ & $(74 \times 22 \mathrm{~m})$ & & & & & & Cüppers, 1979 \\
\hline Augst (1 $1^{\text {er }}$ état $) \ldots$ & $49 \times 22 \mathrm{~m}$ & $4 \times 10$ & $12 \mathrm{~m}$ & & & $\mathrm{I}^{\mathrm{er}}$ s. après J.-C. & $\begin{array}{l}\text { Laur-Belart, 1966, } \\
\text { p. } 43-44\end{array}$ \\
\hline Augst ( $2^{\mathrm{e}}$ état) .... & $65 \times 29 \mathrm{~m}$ & $4 \times 10$ & $18 \mathrm{~m}$ & & & $\begin{array}{l}2^{\mathrm{e}} \text { moitié } \mathrm{II}^{\mathrm{e}} \mathrm{s} \text {. } \\
\text { après } \mathrm{J} .-\mathrm{C} \text {. }\end{array}$ & $\begin{array}{l}\text { Laur-Belart, 1966, } \\
\text { p. } 43-44\end{array}$ \\
\hline
\end{tabular}


vitruvien de Fano, dont la nef centrale est large de 60 pieds ${ }^{26}$. On constate que la basilique de Feurs s'inscrit dans une série de monuments dont la largeur est proche de $24 \mathrm{~m}$ (avec Pompéi, Corinthe, le premier état d'Augst, Clunia, Glanum, Périgueux, Tarragonne et Trieste). Cette analogie se répète dans la division de l'espace interne: dans chacun des exemples nommés, la largeur de la nef centrale (proche de $12 \mathrm{~m}$ ) correspond à environ deux fois celle des portiques latéraux.

La basilique de Feurs s'intègre aussi à la liste, bien fournie, des édifices allongés (longueur supérieure à deux fois la largeur) à nef centrale dont les petits côtés présentent quatre colonnes. Les quatorze colonnes de la longueur donnent à la nef la proportion de 4:1 que l'on retrouve dans deux autres basiliques julio-claudiennes, Tarragonne et Clunia. Dans la mesure de la longueur, nous avons exclu les deux exèdres axiaux (chalcidiques) dont la largeur est équivalente à celle de la nef centrale, ce qui est fréquemment le cas. Ces pièces se retrouvent dans de nombreuses basiliques (Pompéi, Corinthe, Doclea, Clunia) et sont interprétées comme des tribunaux, des aedes Augusti ou des portiques d'entrée. A Feurs, elles ne sont pas intégrées, comme dans les exemples ci-dessus, à la masse rectangulaire de la basilique mais occupent une position d'absides accolées. Il serait possible d'y voir des accès à l'étage, comme c'est le cas à Augst, mais cette fonction semble davantage revenir aux deux petits édicules situés de part et d'autre de la curie, qui sont la principale originalité de la basilique de Feurs. Le massif de maçonnerie repéré dans l'un d'eux pourrait d'ailleurs fort bien être interprété comme un soubassement d'escalier.

La façade occidentale de la basilique n'a pas été dégagée, aussi nous ne pouvons avancer qu'une série d'hypothèses quant à son architecture : ouvertures reprenant le rythme de la colonnade de la nef sur la totalité de la façade, comme à Brescia, ou portes moins nombreuses cantonnées de part et d'autre du centre de la façade, comme à Glanum.

Les entraxes des colonnades de la basilique et des portiques latéraux sont très proches, comme on l'observe également à Ruscino, ce qui renforce l'hypothèse d'un rythme des ouvertures reprenant celui

26 Cetle portée se retrouve dans le dernier état de la basilique d'Augst $(18 \mathrm{~m})$ et se rapproche des deux plus grands espaces couverts en charpente, sans relais intermédiaire, de larchitecture impériale, que sont la basilique de Trajan $(23,7 \mathrm{~m})$ et la basilique de Carthage $(21,9 \mathrm{~m})$ (cf. Gros, 1982. p. 637-641 et note 7 ). de la nef centrale et se reflétant dans celui des portiques.

La position axiale, sur l'un des grands côtés de la basilique, de l'édifice interprété comme la curie, est fréquente sur les fora du Haut Empire (par exemple à Fano, Clunia, Augst, Tarragonne...), même si sa désignation comme lieu de réunion de l'ordo n'est pas toujours clairement affirmée par l'architecture. Cette position l'identifie également à l'aedes Augusti de Vitruve, dont J.-M. David a montré l'intégration, sous des formes diverses, à la basilique (David, 1983).

A Feurs, cet édifice est nettement individualisé par rapport à cette dernière par sa forme, proche de celle d'un temple, et surtout par sa position, qui n'en fait pas, comme à Ruscino, un simple prolongement de la basilique. Les proportions de la curie demeurent néanmoins totalement dépendantes de celles de la basilique. Sa largeur est quasiment identique à celle de la nef centrale, comme à Ruscino (ce qui renforce dans ce cas la continuité des deux espaces).

La curie de Feurs s'inscrit dans une série bien fournie, caractérisée par des dimensions relativement uniformes, qui comprend, entre autres, celles de Timgad, Philippes et Sabratha (Balty, 1962, fig. 1519). Tous ces édifices sans grande originalité architecturale sont formés d'une salle rectangulaire et d'un vestibule, véritable pronaos, qui assimilent leur plan à celui d'un temple. A Feurs, ce vestibule jouait un rôle pratique, celui d'isoler l'assemblée des décurions des activités qui se tenaient dans la basilique, et hiérarchique en conformité avec la vision sacralisée de la vie politique, placée sous l'autorité impériale symbolisée par la statuaire certainement présente en ces lieux. La sacralité de la curie était aussi manifestée par la surélévation de son niveau de circulation vis-à-vis de celui de la basilique, l'escalier d'accès à ce "podium" occupant précisément l'emplacement du vestibule.

\section{LA PLACE CENTRALE ET SES PORTIQUES}

Dans un certain nombre de cas, il y a continuité entre les portiques bordant la place et ceux de la basilique. A Feurs, il est effectivement possible de restituer un rythme des ouvertures de la basilique très proche de celui des portiques. Cet effet peut être renforcé par une largeur analogue, voire identique (cas de Ruscino) des portiques de la place et des déambulatoires de la basilique. A Feurs, ce caractère est moins marqué, les portiques n'ètant pas dans le prolongement des branches latérales du déambulatoire et la largeur dans l'œuvre du portique accusant $6 \mathrm{~m}$. celle du déambulatoire seulement $4.7 \mathrm{~m}$. 
Le plan de masse de la place centrale et de ses portiques est celui d'un carré de $68 \mathrm{~m}$ de côté, construction qui se retrouve à Augst et Trèves (sans tenir compte des cellules extérieures). Le rapport 2:1 $(32,4 \times 62,4 \mathrm{~m})$ des dimensions de la place ellemême, mesuré au pied des emmarchements la reliant aux portiques, se retrouve probablement, mais les dimensions ne sont pas toutes assurées, à Nyon $(31 \times 62 \mathrm{~m})$, Périgueux $(45 \times 90 \mathrm{~m})$ et Amiens $(59 \times 120 \mathrm{~m})$. Dans les cas plus sûrs, les proportions sont : de $2: 3$ à Ruscino $(22,4 \times 33,6 \mathrm{~m})$, proche de $3: 5$ à Augst $(58 \times 33 \mathrm{~m})$ et Conimbriga $(144 \times 84 \mathrm{~m}$ dans l'état augustéen). Dans ce dernier exemple, auquel on pourrait ajouter ceux de Clunia et de Paris, les dimensions de la place obéissent aux préceptes vitruviens. Ce n'est donc pas le cas à Feurs, où elle présente une forme beaucoup plus allongée.

Les cellules et le portique s'inscrivent dans une série bien fournie (comprenant les fora de Conimbriga, Ruscino, Reims, Trèves, Saint-Bertrand-deComminges, Clunia) dont les dimensions varient très peu : largeur entre les axes des cellules comprise entre 4,5 et $6 \mathrm{~m}$, profondeur des cellules entre 6 et $9 \mathrm{~m}$, largeur du portique entre 6,5 et $8 \mathrm{~m}$. Il y a même identité parfaite de toutes les dimensions avec celles relevées sur le forum de Ruscino.

Malgré l'absence de preuve archéologique, c'est sans doute dans les cellules élargies des extrémités des portiques de la place que l'on doit placer les accès à la zone centrale du forum, comme c'est fréquent sur les fora tripartites: Augst, Clunia, Nyon, Benevagienna, Saint-Bertrand-deComminges ...

\section{L'AIRE SACRÉE}

La fouille a montré la continuité de la circulation entre l'aire sacrée et les portiques de la place centrale, cette dernière étant délimitée par un emmarchement situé à environ $2,5 \mathrm{~m}$ en avant des portiques et $4 \mathrm{~m}$ en avant des degrés du temple. Le temple semble donc dégagé au centre de l'aire sacrée, la frontière de cet espace étant seulement manifestée par la transition des portiques de la place centrale vers le cryptoportique. L'élévation présente en effet une solution de continuité, tant dans le rythme des colonnades, dans les niveaux de circulation que dans l'alignement des façades (la façade du cryptoportique est en retrait de $2 \mathrm{~m}$ par rapport à celle des portiques, ce qui engendre un effet de perspective peu satisfaisant, mais que l'on retrouve identique sur plusieurs autres fora, par exemple à Trèves et à Conimbriga, dans le dernier état). Cette rupture nous avait d'ailleurs fait envisager - avant que de nou- velles observations n'infirment cette hyptohèse l'existence d'une clôture de l'espace sacré, comme les récentes fouilles de Nyon viennent de l'attester.

La mise en évidence de l'emprise d'un emmarchement devant le temple prouve que sa façade était tournée vers l'espace central du forum. Elle était aussi légèrement en retrait de la limite orientale de l'aire sacrée. En restituant sur sa face arrière le même espace que sur ses faces latérales, on parviendrait à une longueur de la platée de $27,3 \mathrm{~m}$ (pour une largeur de $14,1 \mathrm{~m}$ ). Les proportions seraient alors précisément celles d'un temple pseudo-périptère (le rapport $1 \div \mathrm{L}$ vaut ici 0,56 , contre 0,55 pour le podium de la Maison Carrée de Nîmes). Ce plan était déjà suggéré par un fragment de colonne (cf. supra, p. 147). Le plan pseudo-périptère (à six colonnes de façade) est déjà attesté en Gaule pour plusieurs temples de forum du début de l'Empire, à SaintBertrand-de-Comminges et Vienne.

Les cryptoportiques du forum de Feurs viennent s'ajouter à la liste, peu fournie, de ceux déjà découverts en Gaule. Nous les avons rassemblés dans un tableau comparatif (tabl. II) en y ajoutant ceux de Conimbriga (époque flavienne), d'Aoste, d'Ampurias, d'Urbisaglia (Delplace, 1979 et 1983) et de Zadar.

Pour les cryptoportiques de Feurs, les données disponibles sont les dimensions en plan des galeries ${ }^{27}$, les accès aux extrémités des branches latérales (vraisemblablement à partir des portiques de la place centrale) et la couverture en plancher (autres exemples à Conimbriga, Nyon, Ampurias, Bavay dans le premier état). Le système de soutènement de cette dernière (piliers axiaux, pilastres) ainsi que celui de l'éclairage des galeries ne nous sont pas connus.

Leur largeur interne s'intègre bien dans la moyenne des exemples retenus (entre 7 et $12 \mathrm{~m}$ ). Plusieurs solutions sont possibles pour la restitution de la hauteur des planchers. Les rares cas oủ la voûte existe encore ne permettent pas de trancher : la hauteur sous clé correspond à la demi-largeur de la galerie (prise dans l'axe des piliers médians) à Arles mais pas à Reims. C'est cependant la solution qui prévaut dans les restitutions d'élévation. Si on l'applique à Feurs, la hauteur restituée serait de $4,7 \mathrm{~m}$, assez proche de celle d'Arles ou de celle restituée à Conimbriga (Alarção, Étienne, 1977, pl. VII) et Ampurias (Aquilué et alii, 1984). Le plafond se situerait donc à la cote 346,3 , et le sol de la porticus triplex surmontant le cryptoportique à un niveau légèrement supérieur compte tenu de la poutraison. Le niveau de

27 L.eur décor peint a été évoqué par (Cl. Allag (1985). 
Tableau II - Dimensions comparées de quelques cryptoportiques de forum (dimensions : dans auvre. la première mesure correspondant à la largeur de la base du $\mathrm{U}$ ).

\begin{tabular}{|c|c|c|c|c|c|c|c|}
\hline Lieu & Dimensions & $\begin{array}{c}\text { Largeur } \\
\text { dans œuvre }\end{array}$ & $\begin{array}{l}\text { Rythme } \\
\text { travées }\end{array}$ & Hauteur & Couverture & Accès & Bibliographie \\
\hline Ampurias ..... & $39 \times 71 \mathrm{~m}$ & $7,6 \mathrm{~m}$ & & $\begin{array}{l}4,7 \mathrm{~m} \\
\text { (restituée) }\end{array}$ & plancher & extrémités galeries & $\begin{array}{l}\text { Aquilué et alii, } \\
1984\end{array}$ \\
\hline Aoste ......... & $87 \times 72 \mathrm{~m}$ & $7,8 \mathrm{~m}$ & & $4,7 \mathrm{~m}$ & voûte & $?$ & $\begin{array}{l}\text { Cavalieri-Manasse } \\
\text { et alii, } 1982\end{array}$ \\
\hline Arles $\ldots \ldots \ldots$ & $58 \times 89 \mathrm{~m}$ & $8,5 \mathrm{~m}$ & $4,2 \mathrm{~m}$ & $4,2 \mathrm{~m}$ & voùte & $\begin{array}{l}\text { extrémités } \\
\text { galeries? }\end{array}$ & Amy, 1973 \\
\hline Bavay ......... & $86 \times 140 \mathrm{~m}$ & $9,6 \mathrm{~m}$ & & & $\begin{array}{l}\text { plancher } \\
\text { (1 er état) }\end{array}$ & milieu galeries & $\begin{array}{l}\text { Hanoune, Muller, } \\
1988\end{array}$ \\
\hline Conimbriga ..... & $44 \times 40 \mathrm{~m}$ & $7,0 \mathrm{~m}$ & $3 \mathrm{~m}$ & $\begin{array}{l}4,3 \mathrm{~m} \\
\text { (restituée) }\end{array}$ & plancher & extrémités galeries & $\begin{array}{l}\text { Alarção, Étienne, } \\
1977\end{array}$ \\
\hline Feurs $\ldots \ldots \ldots$ & $71 \times 60 \mathrm{~m}$ & $9,4 \mathrm{~m}$ & & & plancher & extrémités galeries & \\
\hline Lyon ......... & $83 \times 118 \mathrm{~m}$ & $12,0 \mathrm{~m}$ & $5,2 \mathrm{~m}$ & $\begin{array}{l}6 \text { à } 7 \mathrm{~m} \\
\text { (restituée) }\end{array}$ & voûte & $?$ & $\begin{array}{l}\text { Lasfargues, Le } \\
\text { Glay, } 1980\end{array}$ \\
\hline Nyon .......... & $70 \times 85 \mathrm{~m}$ & $8,0 \mathrm{~m}$ & & & plancher & extrémités galeries & Bridel, 1982a \\
\hline Reims $\ldots \ldots \ldots$ & $93 \times 52 \mathrm{~m}$ & $9,5 \mathrm{~m}$ & $4,3 \mathrm{~m}$ & $5,6 \mathrm{~m}$ & voûte & extrémités galeries & Lallemand, 1969 \\
\hline Trèves ......... & $82 \times 55 \mathrm{~m}$ & $7,5 \mathrm{~m}$ & $3,2 \mathrm{~m}$ & $3,4 \mathrm{~m}$ & voûte & extrémités galeries & Cüppers, 1979 \\
\hline Urbisaglia ..... & $52 \times 42 \mathrm{~m}$ & $7 \mathrm{~m}$ & & & & $?$ & Delplace, 1979 \\
\hline Zadar .......... & $62 \times 71 \mathrm{~m}$ & $9,5 \mathrm{~m}$ & $5,5 \mathrm{~m}$ & & & extrémités galeries & $\begin{array}{l}\text { Ward-Perkins, } \\
1970\end{array}$ \\
\hline
\end{tabular}

l'area sacra se situait au minimum à la surface supérieure du remblaiement repéré $(343,5)$, soit à près de $3 \mathrm{~m}$ en dessous du niveau de circulation du portique. Même si cette dénivellation pouvait être réduite par des remblais que la fouille n'a pas mis en évidence, le niveau du portique paraît très élevé, sa hauteur correspondant sans doute approximativement à celle du podium du temple. C'est le parti pris à Conimbriga dans le sanctuaire flavien, où la dénivellation est de 3,6 m (Alarção, Étienne, 1977, p. 282) et à Reims, où le niveau supérieur du conduit d'assainissement et celui des soupiraux permettent d'envisager une hauteur de de 3,2 à $3,5 \mathrm{~m}$ pour la partie extérieure du cryptoportique (Lallemand, 1969, p. 29-30) ${ }^{28}$. A Aoste (Mollo-Mezzena, 1975-1976, fig. 23), le sol du cryptoportique se trouve au niveau de la place du forum, soit 2,6 $\mathrm{m}$ au-dessous de celui de l'area sacra. Sa partie externe devait mesurer environ $3 \mathrm{~m}$. A Bavay (Will, 1973, fig. 4), Arles (Amy, 1973, fig. 3 et

28 La mème volonté se retrouve, mais en l'absence de cryptoportique, au sanctuaire du Cigognier d Avenches au début du II" s. (Bridel, 1982b, p. 148 et pl. 107).
6) et Trèves (Cüppers, 1979, fig. 8), les coupes publiées révèlent une solution différente : le cryptoportique émerge peu par rapport à l'area sacra, l'accès au portique supérieur pouvant se faire au moyen d'un emmarchement.

Si l'on prend en considération les plans de fora tripartites relativement bien documentés, la présence d'exèdres sur les ailes de la porticus triplex apparait fréquente (ainsi à Saint-Bertrand-deComminges, Benevagienna, Clunia, Trèves, Nyon, Bavay, Arles, Conimbriga, exemples auxquels nous pouvons ajouter les portiques des sanctuaires d'Avenches et de Vaugrenier) mais non systématique : ceux d'Aoste, de Zadar et d'Ampurias en sont dépourvus. Le nombre d'exèdres peut se réduire à une, placée dans l'alignement du grand axe du temple, à trois (une sur chaque aile) ou être multiple (comme à Bavay). La similitude des plans rend tout à fait légitime la restitution sur le forum de Feurs d'une exèdre sur la façade ouest, le rattachant ainsi aux exemples de Trèves et de Conimbriga.

A Feurs, la mesure de la longueur des exèdres n'est pas connue archéologiquement. Le schéma le 
plus simple est représenté par le forum de Nyon dont les trois exèdres présentent la même largeur que la galerie à cryptoportiques, ce qui permet un raccordement simple des toitures au niveau des faîtages, et dans une moindre mesure par celui de Bavay, où la largeur de la galerie se retrouve dans celle des hémicycles des extrémités des branches latérales et dans celle du "vestibule» qui donne accès à la grande salle axiale greffée sur le cryptoportique. Cette solution n'est cependant pas générale. A Conimbriga, les exèdres ont pour largeur la moitié de celle de la galerie, comme à Bavay les petites exèdres et à Bennevagienna les hémicycles des branches latérales. Des exèdres de largeur intermédiaire sont également attestées à Trèves et encore à Benevagienna. C'est la première possibilité qui a été retenue pour la restitution du forum de Feurs. Cette solution est confortée par un autre argument: la galerie étant de même largeur que la curie et quasiment de même largeur que les exèdres de la basilique, l'aspect extérieur des exèdres ainsi restituées, avec leurs parois scandées par des contreforts, comme la curie, répondait à celui de la basilique, équilibrant ainsi la vision d'ensemble du monument. Mieux encore, la situation des exèdres sur les branches latérales du cryptoportique, décalée par rapport à leur axe, peut s'expliquer par le souci de trouver une disposition rigoureusement symétrique à l'ensemble formé par la curie et les deux exèdres de la basilique.

\section{SYNTHÈSE}

I.e forum de Feurs ne présente pas de solutions architecturales originales et, à chaque niveau de comparaison (plan d'ensemble, basilique, aire sacrée), il s'intègre sans difficulté dans des séries déjà bien documentées. Son tracé régulateur est d'une grande simplicité, qui devait probablement se prolonger par celle du décor. Il correspond à la formule la moins élaborée - on serait tenté de dire : la plus rustique - de ce type d'ensemble monumental. Contrairement à Augst, Nyon ou Avenches, elle ne fera pas l'objet de restructuration à une époque ultérieure. La datation que nous avançons pour la construction du forum de Feurs ne paraît pas invraisemblable, puisque le programme qui y est développé était déjà bien connu en Italie dès le début de l'Empire. Qu'elle puisse sembler précoce tient surtout, à notre avis, à l'indigence de la documentation sur les fora de la Gaule interne.

Cette datation permet peut-être de reposer le problème du rapport entre l'édification du forum (tout au moins la décision politique qui a prévalu à sa mise en œuvre) et la mise en place des institutions politico-religieuses dans la civitas. Les exemples sont en effet nombreux qui illustrent les liens unissant le statut juridique, le développement du culte impérial - les deux souvent intimement liés — et l'activité édilitaire. Plus concrètement, la construction du forum implique l'installation d'un culte et d'une administration susceptibles d'occuper ses murs (temple et cellules de la place centrale).

Ia construction du forum de Saint-Bertrand-deComminges semble concorder avec l'octroi du droit latin à la cité des Convènes, sous Auguste. C'est un fait semblable que l'on retrouve peut-être à Amiens sous les Flaviens. Ce phénomène est largement répandu dans la péninsule ibérique, à Sagonte, Bilbilis, Clunia et Belo. Sagonte, pour ne retenir qu'elle, est une civitas foderata de la fin de la République qui devient un municipe de droit latin sous le règne d'Augusle. L'épigraphie atteste la contemporanéité de ce début de la vie municipale (mention d'un duumvir) et la construction de la basilique du forum (Aranegui et alii, 1987, p. 77, 92).

Dans les limites administratives des Trois Gaules à l'époque augustéenne (Goudineau, 1980, p. 97), les fora mis au jour ou localisés appartiennent, en majeure partie, à des capitales de civilates. Mais l'exemple d'Alésia, chef-lieu du pagus des Mandubiens dans la cité des Éduens ou encore les découvertes de Verdes (I oir-et-Cher) (Gallia, 36, 1978, p. 283-284), d'Annecy (Haute-Savoie) (Broise, 1968 ) et d'Eysses (Lot-et-Garonne) ${ }^{29}$, viennent nuancer cette correspondance et nous rappeler que l'existence d'un forum n'est pas obligatoirement liée à une capul civilatis.

Dans le cas de Feurs et de la cité ségusiave, les textes antiques et les documents épigraphiques constituent un dossier peu étoffé où les ambiguïtés sont nombreuses ${ }^{30}$. Le statut de cité libre, acquis

29 Gallia Informations, 1987-1988, 1, p. 148-149. Eysses fut un camp d'époque tibérienne qui aurait pu faire partie d'un quadrillage militaire de la (iaule consécutif à la révolte de 21 (Tassaux, 1984, p. 146-155).

30 Ainsi le texte de Strabon (Géographie IV, I, 11 et 3, 2) dont une interprétation possible conduirait a faire de Lyon, au moins pendant un temps, la capitale des Ségusiaves (sur cette question, consulter les positions contradictoires de Thollard, 1984 et Goudineau, 1989). De mème, laccession de Feurs au rang de colonie découlerait de l'unique témoignage de l'inscription du milliaire de Pommiers (CIL XVII, $346=$ CIL XIII, 8917). Nous devons rappeler que la lecture de ce document épigraphique est loin d'être assuree. 
certainement à une date precoce, est attesté à la fois par Pline ${ }^{31}$ et par des mentions plus tardives sur des milliaires (CIIL XIII, 8861, 8862, 8864).

Les témoignages épigraphiques de fonctions administratives ou religieuses se limitent à celles d'un duumvir sacerdotalis (CIL XIII, 1632) ${ }^{32}$, d'un flamine augustal (CII, XIII, 1629) et d'un sacerdos augusti (CIL XIII, 1642). La seule inscription datée ${ }^{33}$ est la dédicace de Tib. Claudius Capito, fils d'Aruca, qui fit reconstruire en pierre le théâtre d. Feurs sous le règne de Claude (CIL XIII, 1642). Ses Iria nomina et le nom de son père attestent un citoyen de fraîche date. S'il a exercé la fonction de sacerdos augusli, aucune mention n'est faite d'une éventuelle carrière municipale ${ }^{34}$. Doit également être ajoutée à cette brève liste la dédicace à $\mathrm{C}$. Iulius Iullus, princeps des Ségusiaves (CIL XIII, 1645), dont le titre est sans doute seulement la transposition de celui d'une magistrature gauloise. A partir de tels éléments, nous ne pouvons que laisser ouverte la question du statut juridique et de l'octroi éventuel du droit latin aux Ségusiaves ${ }^{35}$.

Une seconde observation se rapporte au culte impérial. Celui-ci a pu revêtir jusqu'au $I^{\mathbf{e}} \mathrm{s}$. un aspect purement local chez les Ségusiaves ${ }^{36}$, suivant

31 La date de la liste donnée par Pline. dans laquelle les Ségusiaves figurent avec ce titre (IIisloria Naluralis, IV, 18, 107 : "Segusiaui liberi in quorum agro colonia Lugdunum"), se situerait vers 20 avant. J.-C.. selon L. Maurin (1978, p. 154). Ce statut n'est accordé à cette époque qu'aux Arvernes, Bituriges Cubi, Gabales, Santons, Leuques, Meldes, Nerviens, Silvanèques, Suessiones et Trévires. Par la suite s'y ajoutèrent les Turones (sous Claude?) et les Vellaves (dans la première moitié du II" s.) (Maurin, 1978, p. 168-173; Goudineau, 1980, p. 314, fig. 240)

32 La gravure originale n'indique que la fonction de duumvir; le terme sacerdolali a été ajouté après coup par une main maladroite au bas de l'inscription.

33 Bien qu'Hirschfeld la situe au $\mathrm{r}^{\mathrm{er}} \mathrm{s}$., à une époque antérieure aux Flaviens, on ne possède pas d'argument pour dater avec précision la dédicace au duumvir Sex. Iulius Lucanus (CIL XIII, 1632).

34 Cf. les tableaux établis par L. Maurin (1978, p. 203).

35 L'existence du duumvirat et du flaminat peut-elle constituer une marque du latium? (sur cette question, cf. Wolff, 1976, p. 48-53; Maurin, 1978, p. 178-183). Le développement du droit latin dans les Trois Gaules est encore mal connu et il ne fut sans doute pas aussi rapide que le pensait Jullian (Jullian, 1929, p. 174).

36 Cf. CIL XIII, 1712. L'inscription, incomplète, permet deux lectures différentes : soit C. Ulattius a été le premier Ségusiave à exercer la charge de prêtre au Confluent, soit il a été le premier de sa cité à qui les Trois Gaules aient élevé une statue. en cela le sort des Lyonnais qui n'auraient pas èté admis au culte fédéral du confluent (Maurin, 1986).

Une inscription forézienne dédiée au numen augusti et au dieu Silvain (CIL XIII, 1640) provient certainement du forum, un des lieux privilégiés pour les réunions des corporations (Waltzing, I, p. 217). Sa datation est difficile. Le développement du culte impérial à Lyon au $\mathrm{II}^{\mathrm{e}} \mathrm{s}$. sous la forme du numina augustorum, souvent associè à des divinités indigènes (Le Glay, 1978, p. 27-28), et l'essor à la mème époque des collèges professionnels dans cette ville (CraccoRuggini, 1978) feraient pencher en faveur du $I^{\circ}$ s., mais en se rappelant que des collèges existent dans les Trois Gaules dès le règne de Tibère (Jullian, 1929, p. 397-398).

Rien ne prouve jusqu'à présent que le temple édifié sur le forum fût à son origine consacré au culte impérial, même si son édification à haute époque ne peut ètre considérée comme un obstacle ${ }^{37}$. D'autres cultes sont connus en pays ségusiave, dont celui de la déesse Segeta, peut-ètre une divinité éponyme du peuple gaulois. Son nom donnait foi à un poidsétalon en bronze découvert à Feurs mème: DEAE. SEG[etae].F[ori].P[ondo].X (CIL XIII, 1641) ${ }^{38}$. Elle était honorée plus particulièrement au sanctuaire thermal d'Aquae Segetae (Moingt, près de Montbrison) mentionné par la Table de Peutinger. Linscription de Bussy-Albieux, à $15 \mathrm{~km}$ à l'ouest de Feurs (CIL XIII, 1646), mentionne encore un temple qui lui était consacré ${ }^{39}$.

Quoi qu'il en soit, les découvertes archéologiques laissent entrevoir une activité urbanistique intense dans la première moitié du $\mathrm{I}^{\mathrm{er}} \mathrm{s}$. : mise en place de la voirie el installation des premiers îlots d'habitat "romanisé" selon un plan concerté et autour d'un ensemble monumental, peut-être encore inachevé, qui affirme la prééminence politique et religieuse de la ville en train de naître. Dans le cas

37 C'est sous Tibère que furent édifiés à Lyon le sanctuaire municipal du clos du Verbe-Incarné et l'amphithéàtre fédéral des Trois Gaules (Lasfargues, Le Glay, 1980, p. 112$113)$.

38 On sait que ces poids-étalons pouvaient ètre conservés dans des lieux publics (forum, macellum), comme l'indique une inscription (CIL XIV, 376) : "Pub. Lucilius Gamala] a aussi donné les poids du marché et les mesures du forum au vin" (cité par Jacques, 1990, p. 131).

39 La déesse Segeta est encore mentionnée par une inscription peinte sur céramique datable du $\mathrm{II}^{\mathrm{I}} \mathrm{s}$. découverte a Roanne (Rodumna), vicus du territoire ségusiave (Poncet, 1981, p. 33). 
précis de Feurs, l'érection de l'ensemble monumental symbolisait d'autant mieux la naissance de la ville que celle-ci portait aussi le nom de Forum, terme issu d'une tradition républicaine dont la Gaule offre très peu d'exemples ${ }^{40}$.

Paul Valette et Vincent Guichard

40 Le choix de l'agglomération gauloise de lieurs comme capitale de cité peut s'expliquer entre autres par sa position par rapport au réseau routier projeté par $\Lambda$ grippa et mis en place à une date encore controversée. La construction à Saintes de l'arc de Germanicus en 18-19 après J.-C. marque en tout cas l'achèvement d'un axe majeur sur lequel Feurs est. situé. Rien ne s'oppose qu'à cette bourgade, conçue comme un Forum routier traditionnel (Ruoff-Väänänen, 1978), soit attaché, lors de l'organisation administrative de la Gaule, le titre de capilale de cité. La construction de l'ensemble monumental sous le règne de Tibère pourrait témoigner de ce nouveau statut.

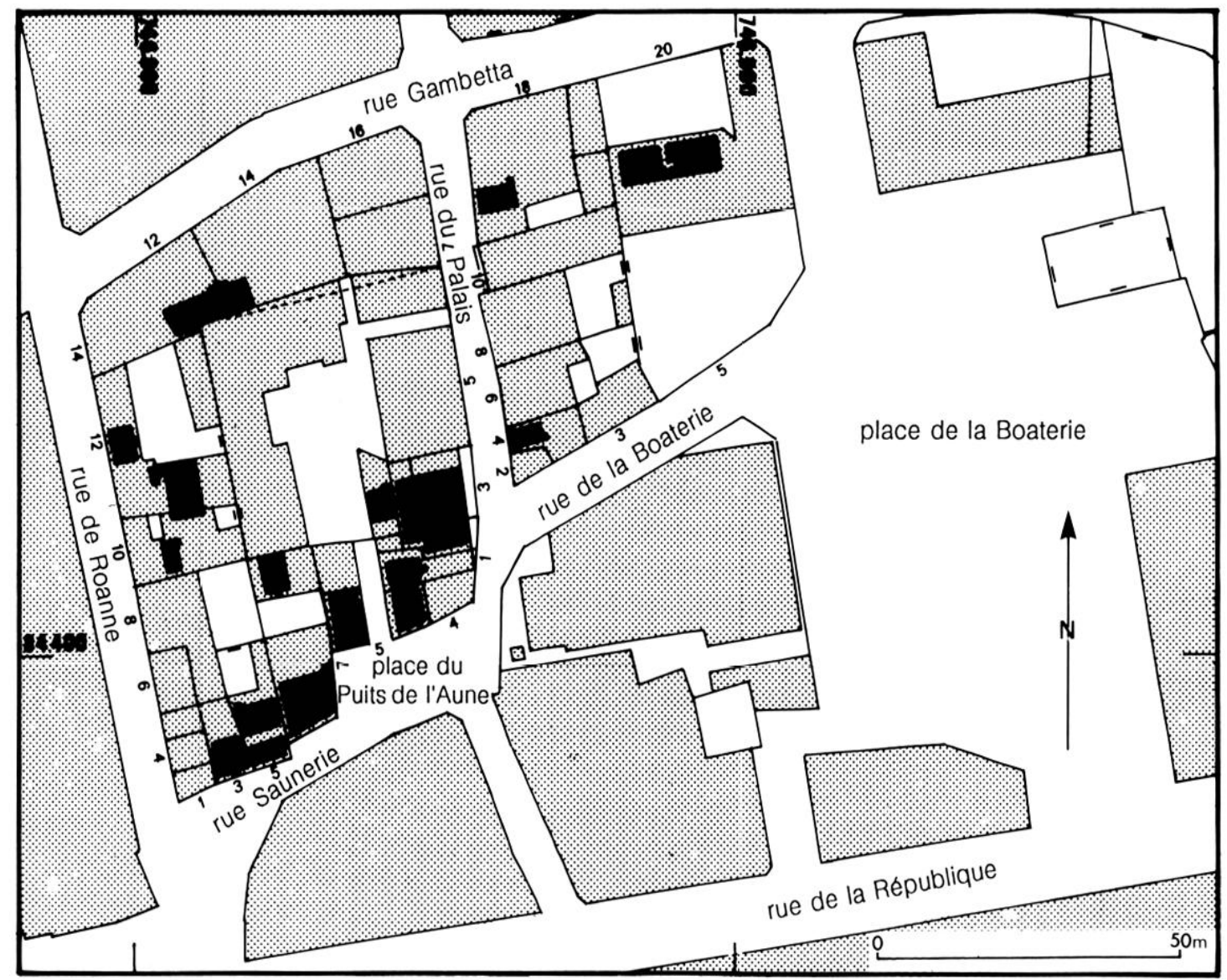

Fig. 29 - Localisation des caves explorées. 


\section{ANNEXE}

\section{Inventaire des caves prospectées}

Figure 29.

\section{Rue de Roanne}

4 : immeuble du $\mathrm{xIX}^{\mathrm{e}}$ s., pas de cave?

6 : immeuble du xixe s.; cave inaccessible; nombreux blocs de calcaire jaune à entroques et blanc en réemploi dans la façade.

8 : immeuble du XIX's.; pas de cave?

10 : immeuble du XIx ${ }^{e} \mathrm{~s}$.; cave du $\mathrm{XIX}^{\mathrm{e}} \mathrm{s}$. entièrement, enduite: même remarque que pour le $n^{\circ} 6$ pour le réemploi en façade

12 : immeuble en partie du XvIII ${ }^{\mathrm{e}} \mathrm{s}$; ; grande cave du XviII ${ }^{\circ}$ s. (?) entièrement enduite, qui pourrait s'appuyer contre le mur extérieur du cryptoportique, et deux petites caves du XIX ${ }^{e}$ s. en façade sur la rue, entièrement enduites.

14 : immeuble du XIX ${ }^{e}$ s.; cave moderne entièrement enduite (non localisée sur le plan).

\section{Rue Gambetta}

12 : immeuble du $\mathrm{XX}^{\mathrm{e}} \mathrm{s}$; ; cave du $\mathrm{XIX}^{\mathrm{c}}$ s. (en fait une ancienne écurie semi-enterrée), accolée contre le rempart du XIV s. qui repose à cet emplacement sur le mur intérieur du cryptoportique (cf. fig. 2, point 7).

14 : immeuble du $\mathrm{xx}^{\mathrm{e}} \mathrm{s}$; ; prolongement de la cave du 12, rue Gambetta, cloisonnée depuis 1981.

16 : immeuble du $\mathrm{xx}^{\mathrm{e}} \mathrm{s}$.; cave moderne non inspectée, qui devrait s'appuyer contre le rempart médiéval reposant à cet emplacement sur le mur extérieur du cryptoportique.

18 : immeuble du $\mathrm{xIX}^{\mathrm{e}} \mathrm{s}$; ; cave ancienne (arc doubleau en plein cintre, en grand appareil), s'appuyant contre le rempart médiéval, qui suit à cet emplacement l'alignement du mur d'enceinte de la place centrale du forum; cependant, aucune maçonnerie antique n'est visible.

20 : immeuble du xviri ${ }^{e}$ s.; caves s'appuyant contre le rempart; maçonneries antérieures à la construction des caves, mais rien ne parait antique.

\section{Rue Saunerie}

1 : immeuble du XIXe s.; pas de cave.

3 : immeuble du $\mathrm{XvI}^{\mathrm{e}} \mathrm{s}$.; cave enduite.
5: immeuble du $\mathrm{xv}^{\mathrm{e}} \mathrm{s}$; une première cave en façade sur la rue, accessible à partir du $n^{0} 3$; une deuxième cave au-delà vers le nord, dont la paroi occidentale s'appuie sur le mur extérieur du cryptoportique (voûte du conduit crevée) (fig. 2, point 8 ).

\section{Place du Puits de l'Aune}

4 : immeuble reconstruit en 1967; une cave ancienne en façade sur la place, entièrement enduite.

5 : dans l'immeuble des $\mathrm{XVII}^{\mathrm{e}-\mathrm{XVII}} \mathrm{J}^{\mathrm{e}} \mathrm{s}$. à gauche de la ruclle d'accès, cave s'appuyant sur le mur intérieur du cryptoportique (fig. 2, point 5); audelà à l'ouest, cave ancienne logée dans l'angle intérieur du cryptoportique, comblée lors de la reconstruction d'un immeuble en 1980 (fig. 2, point 6); à droite de la ruelle, autre cave ancienne, partiellement comblée et actuellement inaccessible; les autres immeubles de la cour intérieure du $\mathrm{n}^{0} \overline{5}$ sont tous récents.

7 : immeuble du XIX s.; cave non voûtée et enduite; un massif de maçonnerie plus ancien visible dans la cave pourrait correspondre au mur extérieur du cryptoportique (fig. 2, point 9).

\section{Rue du Palais}

1: cf. $n^{\circ} 4$, place du Puits de l'Aune.

2 : immeuble du XIX ${ }^{\mathrm{e}} \mathrm{s}$; ; pas de cave.

3: immeuble reconstruit en 1967; grande cave moderne s'appuyant au nord contre le podium du temple (fig. 2, point 12).

4 : immeuble du XIX s. cave en façade sur la rue; emmarchement délimitant la place centrale visible dans la paroi nord (bloc de grand appareil en place).

5 : immeuble du xvine s.; pas de cave.

6 : immeuble du Xixe s.; pas de cave.

7 : immeuble du $\mathrm{xIX}^{\mathrm{e}} \mathrm{s}$.; pas de cave; l'immeuble s'appuie contre le rempart, dont l'élévation est encore conservée sur au moins $3 \mathrm{~m}$ audessus du niveau actuel du sol.

8 : immeuble du xvir1 ${ }^{\text {e }}$ s.; pas de cave en façade sur la rue.

10 : immeuble reconstruit en 1985 ; pas de cave. 


\section{BIBLIOGRAPHIE}

Alarção J., Étienne R.

1977 : Fouilles de Conimbriga, I : L'architecture, Paris, 342 p., $143 \mathrm{pl}$.

\section{Allag C.}

1985 : Le décor peint des cryptoportiques en Gaule, Revue du Nord, 67, p. 9-20.

\section{Amy R.}

1973: Les cryptoportiques d'Arles, in: Les cryploporliques dans l'archilecture romaine. Actes du colloque de Rome, 19-23 avril 1972, Collection de l'École Française de Rome, 14, p. 275-291.

\section{Andreau J.}

1987 : L'espace de la vie financière à Rome, in : L'Urbs. Espace urbain et histoire (I ${ }^{\text {er }} s$. av. J.-C.- $-1 I^{e} s$. ap. J.-C.), Actes du colloque, Rome 8-12 mai 1985, Collection de l'École Française de Rome, 98, p. 157-174.

Aquilué J., Mar R., Nolla J.-M., Ruiz de Arbulo J., Sanmarti E.

1984 : El förum romà d'Empùries, Barcelone, vol. I : texte, vol. II : planches.

Aranegui C. et alii

1987: El foro de Saguntum : La planta arquitectonica, in: Los foros romanos de las provincias occidentales, Madrid, p. 73-97.

Balty J.-C.

1962: Basilique et curie du forum de Glanum: . Vote sur le centre monumental de la ville augustéenne, Latomus, 21, 2, p. 279-319.

Barruol G., Nickels A.

1980 : Le forum et le centre monumental de Ruscino, in : Ruscino, Château-Roussillon, Perpignan (Pyrénées-(Orientales). I : Etat des travaux et recherches en $1975,7^{\mathrm{e}}$ suppl. a la $R A N$, p. 41-66.

Barruol G., Marichal R.

1987 : Le forum de Ruscino, in : Los foros romanos de las provincias occidentales, Madrid, p. 45-54.

Bayard D., Massy J.-L.

1983 : Amiens romain: Samarobriva Ambianorum, Amiens, 374 p., 144 fig.

Bedon R., Chevallier R., Pinon P.

1988: Architecture et urbanisme en Gaule romaine, I: L'architecture et la ville, Paris, 440 p., fig.

Bonnard-Yersin P. et alii

1989: Nyon: La ville et le musée romains, Guides archéologiques de la Suisse, 25, . Nyon, 57 p., 70 fig.

Bossert M., Fuchs M.

1989 : De l'ancien sur le forum d'Avenches, Pro Aventico, 31 , p. 12-108.

\section{Bridel $\mathrm{Ph}$.}

1982a : Le nouveau plan archéologique de Nyon, Archéologie Suisse, 3, p. 178-183.

1982b : Aventicum III: Le sanctuaire du Cigognier, Cahiers d'Archéologie Romande, 22, I,ausanne, vol. I : vi-277 p., pl. 1-67, vol. II : pl. 68-108.
Broise P.

1968 : Découverte d'un édifice public sur le site gallo-romain de Boutae (Les Fins d'Annecy), Latomus, 27, 1, p. 33-34.

Broutin A.

1867 : Ilistoire de la ville de Feurs, Saint-Étienne (3 vol.), réèd. 1984, 544 p., pl.

Cavalieri-Manasse G., Massari G., Rossignani G.

1982 : Piemonte, Valle d'Aosta, Liguria, Lombardia, Guides archéologiques Laterza, Bari, 375 p., fig.

\section{Chartes du Forez}

Guichard G., Neufhourg G. de, Perroy E., I ufour J.-E., Fournial E., Gonon M., Chartes du Forez antérieures au XIV s., Màcon-Paris, 1933-1980 (24 vol.).

Coarelli F.

1982 : Lalium, Guides archéologiques Laterza, Bari.

\section{Cracco-Ruggini $\mathrm{L}$.}

1978 : Les structures de la socièté et de l'èconomie lyonnaises au $\mathrm{II}^{\mathrm{e}}$ s. par rapport à la politique locale et impériale, in : Les Martyrs de Lyon, Paris, p. 65-90.

Crema L.

1959 : Encyclopedia classica, III : Archeologia e storia dell'arte classica : Vol. XII/1, Turin.

\section{Cüppers $\mathrm{H}$.}

1979 : Das römische Forum der Colonia Augusta Treverorum, in: Festschrift 100 .Jahre Rheinisches Landesmuseum Trier, Trierer Grabungen und Forschungen, 14, Mayence, p. 211-269.

David J.-M.

1983 : Le tribunal dans la basilique : Évolution fonctionnelle et symbolique de la République à l'Empire, in : Architecture et société de l'archaisme grec à la fin de la République romaine. Actes du colloque de Rome, 2-4 décembre 1980, Collection de I'École Française de Rome, 66, p. 219-241.

Delplace C.

1979: Les fouilles d'Urbisaglia (province de Macerata en Italie) en 1976-1977, Revue Archéologique, p. 186-188.

1983 : La colonie romaine d'lirbs Salvia et son urbanisation au $\mathrm{I}^{\mathrm{rr}}$ siècle ap. J.-C., MEFRA, 95, 2, p. 761-784.

De Palol P.

1987 : El foro romano de Clunia, in : Los foros romanos de las provincias occidentales, Madrid, p. 153-163.

Desbat A., Mérigoux E.

1979 : Note préliminaire sur la céramique commune de la rue des Farges à Lyon, Figlina, 4, p. 1-17.

Doreau J., Girardy C., Pichonneau J.-F.

1985 : Contribution à l'ètude du forum de Vésone, Aquitania, 3, p. 91-104.

Durand V.

1880: Mémoire inédit de l'abbé J.-F. Duguet, curé de Feurs (1707-1714), Recueils de Mémoires et Documents sur le Forez, 6. Montbrison.

Feugère $\mathbf{M}$.

1985 : Les fibules en Gaule méridionale de la conquête à la fin du $v^{e}$ s. ap. J.-C.., 12e suppl. à la $R A N, 503$ p., 72 fig., 174 pl. 
Fournial E.

1967 : Les villes et l'économie d'echange en forez aux $\mathrm{xIII}^{\circ}$ et XIV siècles, Paris, 1.IV-825) p.. fig. (2 vol.).

\section{Gonon M.}

1974 : Compte des murailles de Feurs, dans Documents linguistiques de France, série franco-provençale, $I:$ Forez, Documents, Études et Répertoires publiés par l'Institut de Recherches et d'Histoire des Textes, 13, Paris.

Gorce J.

1957 : I.es fouilles de la Boaterie à Feurs, Bulletin de la Diana. 35 , p. 60-65.

\section{Goudineau $\mathrm{Chr}$.}

1980 : Les villes de la paix romaine, dans Duoy G. (dir.), IIistoire de la France urbaine, I : La ville antique, Paris, p. 233-391. 1986 : . Notes sur la fondation de Lyon, Gallia, 44, p. 171-173. 1989 : Les textes antiques sur la fondation et la topographie de Lugdunum, in: Coudineau (Chr. (dir.), Aux origines de Lyon, Documents d'Archéologie en Thône-Alpes, 2, Lyon, p. 23-36.

\section{Grenier A.}

1958 : Manuel d'archéologie gallo-romaine, III : L'architecture, $1^{\text {rc }}$ partie, L'urbanisme, les monuments: Capitole, Forum, Temple, Basilique, Paris, 560 p., 187 fig.

\section{Gros P.}

1976 : Hellénisme et romanisation en Gaule narbonnaise, in: Hellenismus in Mitlelitalien, Abhandlungen der Akademie der Wissenschaften in Göttingen, Göttingen, p. 300-314.

1982 : Le forum de la haute ville dans la Carthage romaine d'après les textes et l'archéologie, CRAI, p. 636-658.

1987: Ln programme augusteen: Le centre monumental d'Arles, Jahrbuch des deutschen archäologischen Instituts, 103, p. 339-363.

\section{Gros P., Theodorescu D.}

1985 : Le mur nord du "forum " d'Assise. Ornementation pariétale et specialisation des espaces, MEFRA, 97, 2, p. 879-897.

\section{Gros P., Torelli M.}

1988 : Sloria dell'urbanistica : Il mondo romano, Bari, 596 p.. fig.

\section{Gros P., Varène P.}

1984 : Le forum et la basilique de Glanum : problèmes de chronologie et de restitution, Gallia, 42, p. 21-51.

\section{Guillaumet J.-P.}

1985 : Les fibules du Mont Beuvray, Centre de Recherches sur les Techniques gréco-romaines, 10 , Dijon, $83 \mathrm{p} ., 70 \mathrm{pl}$.

Hallier $\mathrm{G}$.

1984 : Le premier forum de Rougga, Bulletin Archéologique du Comilé des Travaux Historiques et Scientifiques, 17B (1981), p. 101-113.

\section{Hanoune R., Muller R.}

1988: Recherches archéologiques à Bavay I-II, Revue du .Vord, 70, p. 39-56.

\section{Jacques $\mathrm{F}$.}

1990): Les cités de l'occident romain, Paris, 254 p., $2 \mathrm{pl}$.

\section{Jimenez-Salvador J.-L.}

1987a : Arquiteclura forense en la Hispania romana: Bases para su studio, Saragosse, 139 p., 22 fig., 9 photos h.-t.

1987 b : Los modelos en la arquitectura forense de la peninsula ibérica, in: Los foros romanos de las provincias orcidentales, Madrid, p. 173-177.

Jouffroy $\mathbf{H}$.

1986: La construction publique en Italie et dans l'A frique romaine, Groupe de Recherches d'Ilistoire romaine, Etudes et Travaux, 3, Strasbourg.

Jullian C.

1929: Histoire de la Gaule, IV : Le gouvernement de Rome. Paris, $2^{\mathrm{r}}$ éd., $622 \mathrm{p}$.

Kaenel G., Tuor A.

1978 : Les basiliques romaines de .Vyon et de Vidy, Archéologie Suisse, 2, p. 79-83.

Lallemand J. et Lallemand F.

1969 : Les cryptoportiques de Reims, Bulletin de la Société Archéologique Champenoise, 62, p. 18-34.

La Mure J.-M. de

1674 : Histoire universelle civile el ecclésiastique du pays de Forez, Lyon, rééd. Roanne, 1972.

Lasfargues J., Le Glay $\mathbf{M}$.

1980 : Découverte d'un sanctuaire municipal du culte impérial à Lyon, $C R A I$, p. 394-414.

Laur-Belart V.-R.

1966 : Führer durch Augusta Raurica. Bàle. 188 p., fig.

Lefèbvre C., Wagner $P$.

1984 : Metz antique : Remarques sur la connaissance de l'organisation spatiale du fait urbain, in: Les villes de la Caule belgique sous le Haut Empire. Actes du colloque de Saint-Riquier, 1982, Amiens ( = Revue Archéologique de Picardie, 3/4), p. 149169.

\section{Le Gall J.}

1978 : Que faisait-on sur un forum?, in : Forum et Plaza Major dans le monde hispanique, Publications de la Casa Velasquez, Paris, p. 23-26.

\section{Le Glay $\mathrm{M}$.}

1978 : Le culte impérial à Lyon au $\mathrm{II}^{\mathrm{F}} \mathrm{s}$, in : Les .Marlyrs de Lyon, Paris, p. 19-29.

Le Glay M., Audin A.

1976 : Récentes découvertes épigraphiques à Lyon, in : Notes d'épigraphie et d'archéologie lyonnaises, Lyon, p. 5-44.

Loustaud J.-P.

1981 : Le forum de Limoges : Premières approches, 1976-1980. Bulletin de la Société Archéologique el IIistorique du Limousin. 108 , p. 40-53.

\section{Mangin M., Bénard J.}

1985 : Les étapes de la romanisation d'une agglomération indigène du centre-est de la Gaule : L'exemple d'Alésia, in : Les débuls de l'urbanisation en Gaule et dans les provinces voisines, Cæsarodunum, 20, Tours, p. 103-105.

Mar R., Ruiz de Arbulo J.

1987 : La basílica de la colonia Tarraco: Una nueva interpretación del llamado Foro bajo de Tarragona, in : Los foros romanos de las provincias occidentales, Madrid, p. 31-44.

\section{Maurin L.}

1978 : Saintes antique, des origines à la fin du $v^{p}$ s. après. JésusChrist, Saintes.

1986 : Gaulois et. Lyonnais, in : Hommage à Robert Élienne. Revue des Études Anciennes, 88, p. 109-124. 


\section{Mertens J.}

1973 : Le "cryptoportique" d'Ordona (Apulia), in : Les cryptoportiques dans l'archileclure romaine. Acles du colloque de Rome. 19-23 avril 1972. Collection de I'École Française de Rome. 14. p. 187-205.

\section{Mollo-Mezzena R.}

1975-1976: Augusta Pratoria. Aggiomamento sulle conoscenze archeologiche della città e del suo territorio, Revue d'Études Ligures, 41-42, 1-4, p. 147-257.

Ohr K.-F.

1977: Die Basilika in Pompeji : Untersuchungsergebnisse 1966-1971, Cronache Pompeiane, 3, p. 17-39.

Olivier A., Rogers G.

1978 : Le monument de Vaugrenier (Alpes-Maritimes), RAN, 11, p. 143-193.

Périn $P$.

1984 : Le forum, dans Fleury M. (dir.), Lalèce: Paris de Cèsar à Clovis, catalogue de l'exposition, Paris, p. 154-160.

\section{Poncet $\mathbf{J}$.}

1981 : La nécropole gallo-romaine de Roanne. Cahiers Archéologiques de la Loire, 1, p. 27-38.

Poncet J., Aulas C., Vaginay M.

1979: Deux fosses augustéennes de Hoanne, Bulletin des Groupes de Recherches Archéologiques de la Loire, p. 77-107.

\section{Poncins Comte de}

1888: Inscription mentionnant la construction du théatre antique de Feurs. Bulletin de la Diana, 4, p. 272-276.

Rémy B.

1972: Deux inscriptions de Feurs retrouvées. Bulletin de la Diana, 42, p. :203-205.

Rivet $\mathbf{L}$.

1987 : Le forum d'Aquae Sextie, in : Los foros romanos de las provincias occidentales, Madrid, p. 185-190.

\section{Roth-Congès $\mathrm{A}$.}

1987a : Fouilles et recherches récentes sur le forum de Glanum, in: Los foros romanos de las provincias occidentales. Madrid, p. 191-201.

1987b : L'hypothèse d'une basilique à deux nefs a Conimbriga et les transformations du forum, MEFRA, 99, 2, p. 711-751.

\section{Roux J.}

1851 : Recherches sur le Forum Segusiavorum et l'origine galloromaine de la ville de Feurs, I.yon, 100 p.. XXVII pl. h.-t.

\section{Ruoff-Väänänen E.}

1978 : Studies on the italian fora, Historia, Einzelschriften, 32, Wiesbaden, 81 p., 2 pl.

Sablayrolles R., Sillières P.

1987 : Le temple du forum a saint-Bertrand-de-Comminges, Dossiers d'Histoire el d'Archéologie, 120, p. 46-47.

Salviat F.

1979 : Tibère, Drusus et le forum d'Avignon, Revue Archéologique, p. 259-268.

\section{Stern H., Blanchard-Lemée M.}

1975 : Recueil général des mosaïques de Gaule, II : Province de Lyonnaise : 2, $10^{\circ}$ suppl. à Gallia, n 166 , pl. II et III.

Sticotti P.

1913: Die römische Sladt Doclea in Montenegro, Vienne. 226 col., pl.

Tassaux D. et Tassaux F.

1984: Aulnay-de-Saintonge : un camp militaire augustotibérien en Aquitaine, Aquitania, 2, p. 105-157.

Thollard P.

1984: Strabon, Lyon, Vienne et les Ségusiaves, $R A N, 17$. p. 115-122.

\section{Vaginay M., Guichard V.}

1988 : L'habital gaulois de Feurs (Loire), Documents d'Archéologie Française, 14, Paris, Maison des Sciences de l'llomme, 199 p., 153 fig.

Vaginay M., Valette P.

1982 : Recherches sur les origines de l'urbanisme antique de Feurs, Cahiers Archéologiques de la Loire, 2, p. 39-72.

Valette P.

1981 : Un puits gallo-romain, élément de datation pour la construction du forum de Feurs, Cahiers Archéologiques de la Loire, 1, p. 55-60.

1983 : Le Ilaut Moyen Age en Forez: État de la question, Cahiers Archéologiques de la Loire, 3, p. 73-78.

1989 : Découverte de nouveaux témoignages de la vie religieuse a Feurs dans l'Antiquité, Cahiers Archéologiques de la Loire, 6, p. 69-80.

Vigneron B.

1986: Metz antique, Metz, 306 p., fig.

Waltzing J.-P.

1895-1900 : Etude historique sur les corporations professionnelles chez les Romains depuis les origines jusqu'à la chute de l'Empire d'Occident, rééd. Rome, 1968 (4 vol.).

\section{Ward-Perkins J.-B.}

1970 : From Republic to Empire: Reflexions on Early Provincial Architecture of the Roman West. Journal of Roman Sludies, 60, p. 1-19.

Wiblé F.

1981: Forum Claudii Vallensium: La ville romaine de Martigny. (iuides archéologiques de la Suisse, 17, 40 p., 62 fig. Will E.

1973 : Les cryptoportiques de forum de la Gaule, in : Les cryploporliques dans l'architecture romaine. Actes du colloque de Rome, 19-23 auril 1972, Collection de l'École Française de Rome, 14, p. 325-342.

Wolff $\mathrm{H}$.

1976 : Kriterien für latinische und römische Städte in Gallien und Germanien und die "Verfassung" der gallischen Stammesgemeinden, Bonner Jahrbücher, 176, p. 14-121.

Zaccaria C.

1988 : Problemi epigrafici del foro di Trieste, MFFRA, 100), 1, p. 63-85. 